Universidade de São Paulo

Instituto de Física

\title{
ESTRUTURA ELETRÔNICA DE ISOLANTES TOPOLÓGICOS EM DUAS E TRES DIMENSÕES
}

\section{Leandro Seixas Rocha}

Tese de doutorado apresentada ao Instituto de Física para a obtenção do título de Doutor em Ciências.

Orientador: Prof. Dr. Adalberto Fazzio

\section{Comissão examinadora:}

- Prof. Dr. Adalberto Fazzio (IF/USP)

- Prof. Dr. José Fernando Diniz Chubaci (IF/USP)

- Prof. Dr. Mário José de Oliveira (IF/USP)

- Prof. Dr. Vágner Eustáquio de Carvalho (ICEx/UFMG)

- Dr. Harry Westfahl Junior (LNLS/CNPEM)

São Paulo

2014 


\section{FICHA CATALOGRÁFICA}

Preparada pelo Serviço de Biblioteca e Informação do Instituto de Física da Universidade de São Paulo

Rocha, Leandro Seixas

Estrutura eletrônica de isolantes topológicos em duas

e três dimensões. São Paulo, 2014.

Tese (Doutorado) - Universidade de São Paulo. Instituto de Física. Depto. Física dos Materiais e Mecânica.

Orientador: Prof. Dr. Adalberto Fazzio

Área de Concentração: Estrutura Eletrônica.

Unitermos: 1. Física do estado sólido ; 2. Propriedades dos sólidos; 3. Superfície física; 4. Física computacional; 5. Física teórica.

USP/IF/SBI-044/2014 


\section{Agradecimentos}

Agradeço primeiramente à minha família, em especial à minha mãe, Janne, pelo apoio e presença durante boa parte da minha estadia em São Paulo.

Agradeço ao meu orientador, Prof. Dr. Adalberto Fazzio, pelas valorosas discussões sobre aspectos gerais em física e sobre os sistemas estudados nesta tese de doutorado. Ao Prof. Shengbai Zhang, que me orientou no meu período sanduíche realizado no Rensselaer Polytechnic Institute (RPI). Aos Profs. Antônio José Roque da Silva, Tomé Mauro Schmidt e Roberto Hiroki Miwa, pelas importantes discussões sobre física, em especial sobre os isolantes topológicos.

Agradeços aos membros do grupo SAMPA e aos seus colaboradores, principalmente ao Padilha, ao Matheus, ao Renato, ao James, ao Rodrigo, ao Pedro, ao Alberto, ao Carlos, ao Leonardo, ao Alexsandro, ao Ernesto e ao Vagner. Também agradeço à Marisa, pelo suporte dado ao grupo.

Finalmente agradeço ao CNPq pelo apoio financeiro dado pela concessão da bolsa doutorado, e ao CENAPAD/Unicamp pelo recurso computacional utilizado nessa tese. 
All human power is a compound of time and patience.

Honoré de Balzac

The boundaries of bodies are the least of all things.

Leonardo da Vinci 
Nessa tese de doutorado apresentamos um estudo da estrutura eletrônica de materiais isolantes topológicos. A teoria fundamental dos isolantes topológicos foi abordada através de invariantes topológicos $\mathbf{Z}_{2}$, assim como os seus métodos para o cálculo desses invariantes topológicos e as consequências da topologia de bandas não-trivial. Assim como as propriedades atomísticas e energéticas, as propriedades eletrônicas de alguns isolantes topológicos foram calculadas através de métodos de primeiros princípios baseados na Teoria do Funcional da Densidade. Apresentamos nessa tese o estudo de quatro sistemas de interesse físico: (1) Em isolantes topológicos do tipo $\mathrm{Bi}_{2} \mathrm{Se}_{3}$ e $\mathrm{Bi}_{2} \mathrm{Te}_{3}$ com falhas de empilhamentos, encontramos que o $\mathrm{Bi}_{2} \mathrm{Te}_{3}$ com falhas de empilhamentos apresentam estados metálicos na região do defeito; (2) Na interface $\mathrm{Bi}_{2} \mathrm{Se}_{3} / \mathrm{GaAs}$ com tratamento de Se na região do GaAs, encontramos que a interação entre o cone de Dirac do $\mathrm{Bi}_{2} \mathrm{Se}_{3}$ com a banda de valência do GaAs abre um gap de energia no ponto $\bar{\Gamma}$; (3) Em nanoestradas de germaneno imersas em germanano com interfaces zigzag, encontramos que a partir de uma largura crítica podemos observar o efeito Hall quântico de spin; e (4) nas ligas desordenadas hexagonais de $\mathrm{Si}_{x} \mathrm{Ge}_{1-x}$ em duas dimensões, o sistema desordenado compartilha a mesma topologia de bandas do siliceno e do germaneno, 
enquanto que a liga ordenada $\mathrm{Si}_{0.5} \mathrm{Ge}_{0.5}$ é um isolante trivial. As estruturas eletrônicas desses sistemas foram investigadas no intuito de entender as consequências físicas da topologia de bandas nãotrivial nos estados de Bloch de bulk e de superfícies/interfaces.

Palavras-chaves: estrutura eletrônica, isolantes topológicos, topologia de bandas, cone de Dirac. 
In this doctoral thesis we present a study of the electronic structure of topological insulators materials. The fundamental theory of topological insulators was addressed through the $\mathbf{Z}_{2}$ topological invariants, as well as their methods to calculate these topological invariants and the consequences of non-trivial band topology. Just as atomistic and energetic properties, the electronic properties of some topological insulators were calculated using first-principles methods based upon Density Functional Theory. We present in this thesis the study of four systems of physical interest: (1) In topological insulators like $\mathrm{Bi}_{2} \mathrm{Se}_{3}$ and $\mathrm{Bi}_{2} \mathrm{Te}_{3}$ with stacking faults, we found that the $\mathrm{Bi}_{2} \mathrm{Te}_{3}$ with stacking faults presents metallic

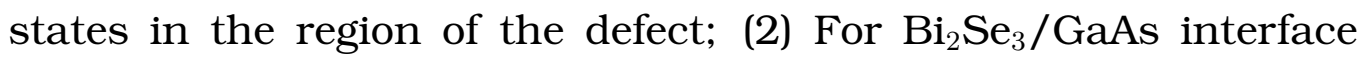
with Se-treatment in the GaAs region, we found that the interaction between the Dirac cone of the $\mathrm{Bi}_{2} \mathrm{Se}_{3}$ and the valence band of the GaAs opens a bandgap at the $\bar{\Gamma}$-point; (3) In germanene nanoroads embedded on germanane with zigzag interfaces/edge, we found that from a critical width we can observe the quantum spin Hall effect; and (4) For $\mathrm{Si}_{x} \mathrm{Ge}_{1-x}$ two-dimensional hexagonal disordered alloy, the system shares the same non-trivial band topology of the silicene and germanene, while the ordered alloy $\mathrm{Si}_{0.5} \mathrm{Ge}_{0.5}$ is a trivial insulator. The electronic structures of these systems were 
investigated in order to understand the physical consequences of non-trivial band topology in the bulk and surfaces/interfaces Bloch states.

Keywords: electronic structure, topological insulators, band topology, Dirac cone. 
$\begin{array}{ll}\text { Agradecimentos } & \text { iii }\end{array}$

Epigrafe $\quad$ iv

Resumo v v

Abstract vii

I Fundamentos xiii

1 Introdução 1

2 Isolantes topológicos 9

2.1 Introdução . . . . . . . . . . . . . . . 9

2.2 Teoria de bandas . . . . . . . . . . . . 11

2.2.1 Hamiltoniano de Bloch . . . . . . . . . 11

2.2.2 Rede recíproca e zona de Brillouin . . . . . . 13

2.2.3 Estrutura de bandas . . . . . . . . . . 15

2.3 Teoria de Berry . . . . . . . . . . . . . . . 17

2.3.1 Transporte paralelo . . . . . . . . . 17

2.3.2 Conexão e curvatura de Berry . . . . . . . 17 
2.4 Simetria de reversão temporal . . . . . . . . . . . . 20

2.5 Topologia de bandas $Z_{2} \ldots \ldots \ldots \ldots$

2.5.1 Invariante $Z_{2}$ para materiais centrossimétricos 26

2.5.2 Invariantes $Z_{2}$ em três dimensões . . . . . . . 29

2.6 Exemplos de isolantes topológicos . . . . . . . . . 30

2.6.1 Siliceno . . . . . . . . . . . . 31

$2.6 .2 \mathrm{Bi}_{2} \mathrm{Se}_{3}, \mathrm{Bi}_{2} \mathrm{Te}_{3}$ e $\mathrm{Sb}_{2} \mathrm{Te}_{3} \ldots \ldots \ldots 32$

II Resultados

3 Falhas de empilhamentos em $\mathrm{Bi}_{2} \mathrm{Se}_{3} \mathbf{e ~ B i}_{2} \mathbf{T e}_{3} \quad 37$

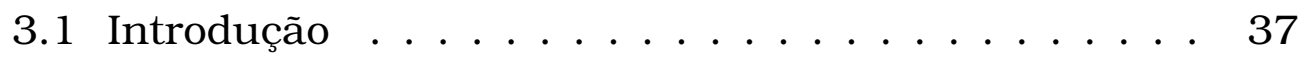

3.2 Propriedades estruturais . . . . . . . . . . . . . 38

3.3 Propriedades eletrônicas . . . . . . . . . . . . . . 42

3.4 Falhas de empilhamentos próximas às superfícies . . 45

3.5 Conclusão . . . . . . . . . . . . . . . . . . 45

4 Interfaces entre isolantes topológicos e semicondutores 49

4.1 Introdução . . . . . . . . . . . . . . . 49

4.2 Modelo Hamiltoniano efetivo . . . . . . . . . . . . 51

4.3 Cálculos de primeiros princípios . . . . . . . . . . 55

4.4 Interação TI/S . . . . . . . . . . . . . . . 61

4.5 Conclusão . . . . . . . . . . . . . . . . . . 67

5 Nanoestradas de germaneno 69

5.1 Introdução . . . . . . . . . . . . . . . . . 69

5.2 O germaneno e o germanano . . . . . . . . . 70

5.3 Dissociação de hidrogênio em germanano . . . . . . 75

5.4 Nanoestradas de germaneno imersas em germanano . 75

5.5 Conclusão . . . . . . . . . . . . . . . . 82

6 Ligas desordenadas $\mathrm{Si}_{x} \mathrm{Ge}_{1-x}$ em duas dimensões 83

6.1 Introdução . . . . . . . . . . . . . . . 83

6.2 Ligas desordenadas e SQS . . . . . . . . . . . . 85 
6.3 Estrutura eletrônica das ligas $\mathrm{Si}_{x} \mathrm{Ge}_{1-x}$ em duas di-

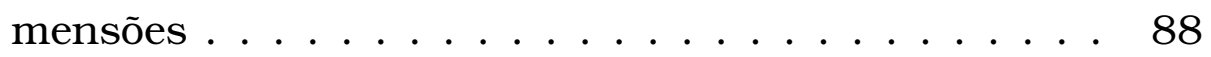

6.4 Efeito de campo elétrico . . . . . . . . . . . 91

6.5 Conclusões . . . . . . . . . . . . . . . 93

7 Conclusões gerais $\quad 95$

$\begin{array}{ll}\text { III Apêndices } & 97\end{array}$

A Teoria do Funcional da Densidade 99

A.1 Teoremas de Hohenberg-Kohn . . . . . . . . . 100

A.2 Aproximação de Thomas-Fermi . . . . . . . . . . 102

A.2.1 Modificações da aproximação de Thomas-Fermi 103

A.3 Equações de Kohn-Sham . . . . . . . . . . . . . 105

A.4 Funcionais de troca e correlação . . . . . . . . . . 106

A.4.1 Aproximação da densidade local (LDA) . . . . . 106

A.4.2 Aproximação do gradiente generalizado (GGA) . 107

B Equação de Dirac e acoplamento spin-órbita 109

B.1 Equação de Dirac . . . . . . . . . . . . . . . . 110

B.1.1 Limite de baixas energias . . . . . . . . . . . 112

B.2 Acoplamento spin-órbita . . . . . . . . . . . . 113

C Invariantes $Z_{2}$ em ondas planas 115

C. 1 Invariante $\mathbf{Z}_{2}$ centrossimétrico . . . . . . . . . 115

C.2 Invariante $\mathbf{Z}_{2}$ sem simetria de inversão . . . . . . . 116

$\begin{array}{lr}\text { D Lista de publicações } & 119\end{array}$ 


\section{Parte I}

\section{Fundamentos}





\section{Introdução}

God created the solids, the devil their surfaces.

Wolfgang Pauli

A história dos isolantes topológicos tem origem no estudo do efeito Hall. Esse efeito surgiu de um erro cometido pelo James C. Maxwell em seu livro "Treatise on Electricity and Magnetism", publicado em 1873 [1]. Ao discutir sobre a deflexão de uma corrente elétrica por um campo magnético, Maxwell escreveu que a força mecânica agia diretamente no condutor, e não na corrente elétrica.

Em 1878, duvidando da afirmação de Maxwell, o estudante Edwin Hall fez um experimento com campo magnético atuando perpendicularmente sobre uma folha fina de ouro. O campo magnético defletiu a corrente elétrica para as bordas da folha, acumulando cargas elétricas nessa região. Quando o acúmulo de cargas elétricas se estabilizou, foi possível medir uma tensão elétrica entre as bordas. A partir dessa tensão elétrica entre bordas $\left(V_{H}\right) \mathrm{e}$ da corrente elétrica $(I)$ que fluia na folha fina de ouro, foi possivel definir a resistência

$$
R_{H}=\frac{V_{H}}{I},
$$

que ficou conhecida mais tarde como resistência Hall. A existên- 
cia desse efeito medido através da resistência Hall mostrou que a afirmação de Maxwell estava errada, e que a força magnética atuava diretamente sobre a corrente elétrica.

Além disso, a resistência Hall mostrou-se uma importante propriedade física na caracterização de materiais. Cada material apresenta resistência Hall diferente, chegando até a inverter o seu sinal de acordo com o tipo de portador de carga conduzido pelo material.

Do modelo de Drude [2], podemos entender a teoria clássica microscópica do efeito Hall. Para um sistema com densidade de elétrons $n$, a resistência Hall é

$$
R_{H}=-\frac{1}{n e}
$$

onde $e$ é a carga elementar.

Para materiais semicondutores que conduzem tanto por elétrons como por buracos, a resistência Hall torna-se

$$
R_{H}=\frac{p \mu_{h}^{2}-n \mu_{e}^{2}}{e\left(p \mu_{h}+n \mu_{e}\right)^{2}},
$$

onde $n(p)$ é a densidade de elétrons (buracos), e $\mu_{e}\left(\mu_{h}\right)$ é a mobilidade de elétrons (buracos) dada por $\mu_{i}=v_{d, i} / E$, onde $v_{d, i}$ é a velocidade do portador de carga $i$ quando sob efeito do campo elétrico de intensidade $E$.

Com o advento da mecânica quântica a partir da década de 20, foi possivel descrever corretamente as propriedades eletrônicas e de transporte dos materiais. A descrição dos estados quânticos eletrônicos nos sólidos permitiu a classificação dos materiais em isolantes, semicondutores ou metais. Também foi possivel descrever os estados eletrônicos na presença de um campo magnético. A quantização das órbitas ciclotrônicas deram origem à níveis discretos de energias chamados de niveis de Landau. Entretanto, a observação desse efeito quântico está atrelada à condições de baixíssimas temperaturas e campos magnéticos de altas intensidades. 
Quanto temos um material submetido a baixíssimas temperaturas (até 1,5 K) e campos magnéticos de altas intensidades (acima de 13 T), os níveis de Landau do material dão origem ao efeito Hall quântico. Em 1980, Klaus von Klitzing e colaboradores estudaram o efeito de campos magnéticos de até $18 \mathrm{~T}$ e temperaturas de 1,5 K em dispositivos MOSFET de Si para observar a quantização do efeito Hall [3]. A camada de inversão do MOSFET agiu como um metal que substituiu a folha fina de ouro do experimento original de Hall.

A condutância Hall (inverso da resistência Hall) medida, demonstrou patamares de constância que é explicada pela teoria quântica. A condutância Hall no efeito Hall quântico é

$$
\sigma_{x y}=\nu \frac{e^{2}}{h}
$$

onde $h$ é a constante de Planck, e $\nu$ é um número inteiro que depende da topologia de bandas sob o efeito do campo magnético [3-8]. O número $\nu$ foi posteriormente chamado de invariante TKNN devido a teoria topológica proposta por Thouless, Kohmoto, Nightingale e den Nijs em 1982 [4]. A teoria TKNN é formulada de forma que o invariante TKNN é dado por uma integral da curvatura de Berry na zona de Brillouin, dividido por $2 \pi$, como será descrito no Capítulo 2. Esse invariante é uma propriedade topológica da teoria de bandas, e dessa forma, são robustos por perturbações no sistema. Como mostrado por Halperin [6], essa propriedade topológica no bulk do material se manifesta nas bordas através de estados metálicos topologicamente protegidos. A correspondência entre os estados topológicos de bulk da teoria TKNN e dos estados metálicos de Halperin foi demonstrada através da correspondência bulk-borda [7].

Outra manifestação do efeito Hall é o efeito Hall de spin, previsto em 1971 por Dyakonov e Perel [9]. Nesse efeito, as correntes de bordas são polarizadas em spin devido ao acúmulo de spins 
gerados por espalhamentos da corrente elétrica no bulk. Quando a corrente elétrica é invertida, as orientações de spin dos estados de bordas também são invertidas. O acúmulo de spins devido ao espalhamento assimétrico entre spin up e spin down é chamado de efeito Hall de spin extrínseco. Em 2003 [10] foi previsto que o acoplamento spin-órbita do material poderia gerar um efeito Hall de spin intrínseco, sem a dependência de espalhamentos assimétricos de centros espalhadores no bulk. O efeito Hall de spin foi observado em 2004 em filmes finos de GaAs e InGaAs [11].

Em 2005, estudando o efeito do acoplamento spin-órbita no grafeno, Kane e Mele [12] previram o efeito Hall quântico de spin. Esse efeito é essencialmente dois efeitos Hall quântico para elétrons com quiralidades opostas. A partir da simetria de reversão temporal, podemos relacionar os elétrons em pares de Kramers, que dão origem a essas quiralidades opostas. $\mathrm{O}$ acoplamento spinórbita nesse efeito deve ser grande o suficiente para que ocorra uma inversão dos caráteres da banda de valência com a banda de condução.

Embora tenha sido previsto primeiramente para o grafeno, o pequeno acoplamento spin-órbita desse material introduz dificuldades para a observação desse efeito em condições realistas que podem ser alcançadas em laboratório. Em 2006, Bernevig, Hughes e Zhang [13] previram que o efeito Hall quântico de spin poderia ser observado em poços quânticos de CdTe/HgTe. Os materiais CdTe e HgTe possuem bandas de valência e de condução com representações de simetrias invertidas [ver Fig. 1.1(a)]. Dessa forma, os efeito de confinamento nos poços de HgTe poderiam apresentar sub-bandas $E 1$ e $H 1$ em diferentes níveis de energias dependendo das larguras $d$ dos poços quânticos. As energias das sub-bandas $E 1$ e $H 1$ podem se inverter a partir de uma largura crítica $d_{c} \approx 64$ Å, como mostrado na Fig. 1.1(b).

Esse efeito foi observado experimentalmente em 2007 por König et al. [14] através de medições da condutância para várias larguras 
A
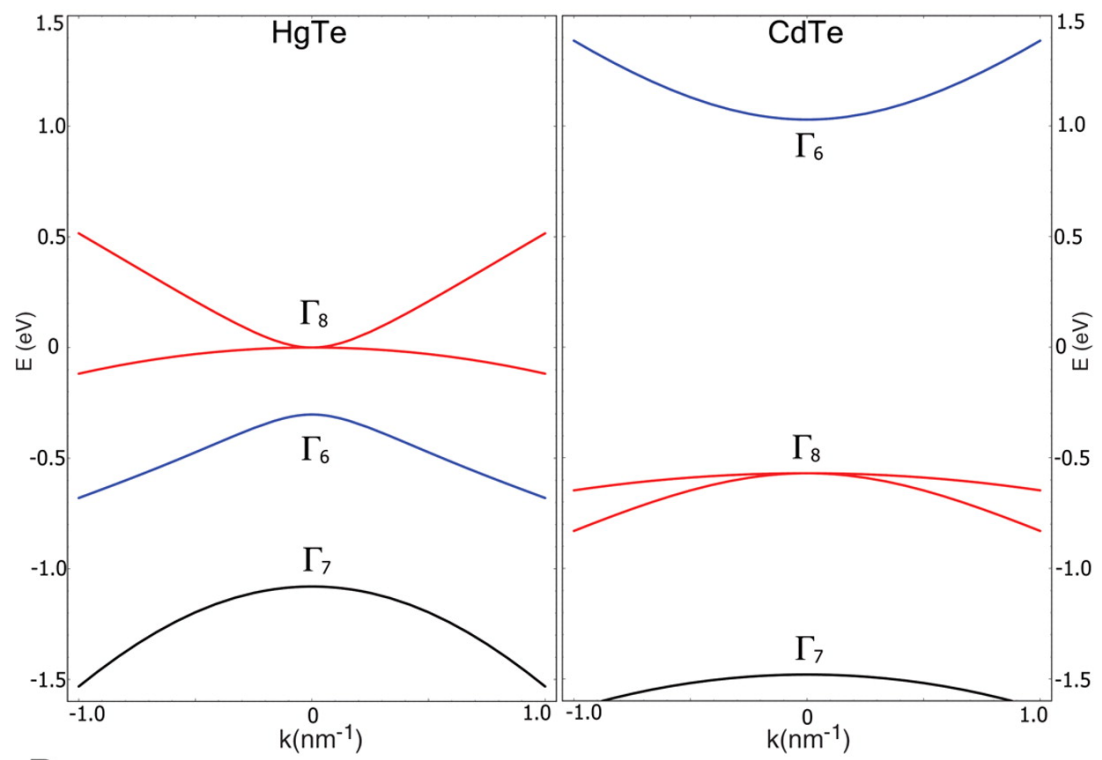

B
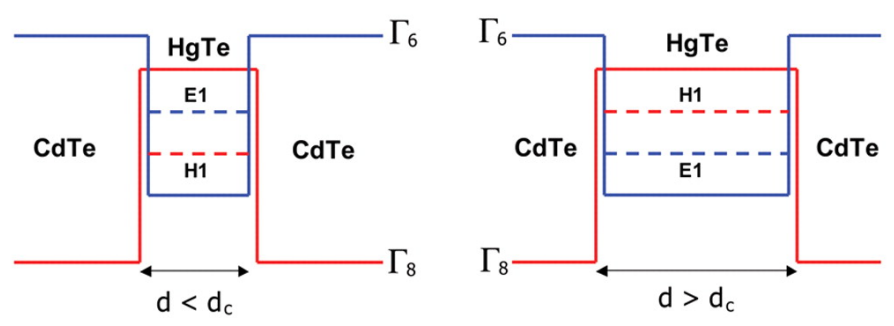

Figura 1.1: (a) Estruturas de bandas do HgTe (painél à esquerda) e CdTe (painél à direita). (b) Efeito de confinamento das sub-bandas $E 1$ e $H 1$ no poço quântico de HgTe. Figura extraída da referência [13]. 
de poços quânticos de HgTe, como mostrado na Fig. 1.2.

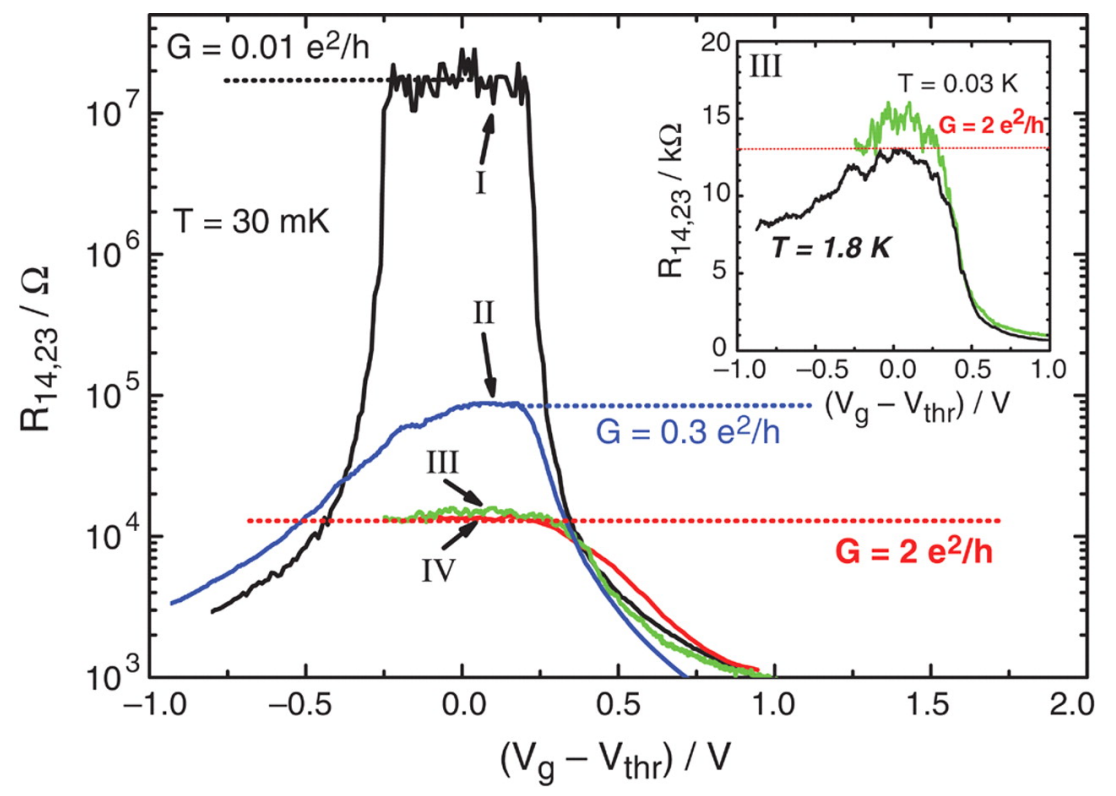

Figura 1.2: Resistência Hall para várias larguras de poços quânticos de CdTe/HgTe. Figura extraída da referência [14].

Em 2007, Moore e Balents generalizaram o conceito de efeito Hall quântico de spin para materiais em três dimensões [15]. Esses materiais, batizados de isolantes topológicos (TI, acrônimo do termo em inglês Topological Insulators), possuem bulk isolante e superfície metálica com polarizações de spin com origem na topologia de bandas de bulk. Tais materiais também foram caracterizados por quatro invariantes topológicos $\mathrm{Z}_{2}$ (ver Capítulo 2). $\mathrm{O}$ primeiro isolante topológico em três dimensões previsto foi o $\mathrm{Bi}_{1-x} \mathrm{Sb}_{x}$ [16], que foi confirmado experimentalmente em 2008 por Hsieh et al. [17].

A previsão [18] e observação [19,20] dos isolantes topológicos $\mathrm{Bi}_{2} \mathrm{Se}_{3}, \mathrm{Bi}_{2} \mathrm{Te}_{3}$, e $\mathrm{Sb}_{2} \mathrm{Te}_{3}$ formaram uma segunda geração de isolantes topológicos 3D [21]. A partir desses materiais, o estudo teórico e experimental dos isolantes topológicos tornaram-se mais acessíveis. Foram nesses materiais que partimos para o estudo de estrutura eletrônica e efeitos de topologias de bandas dos isolantes 
topológicos.

Desde a descoberta dos isolantes topológicos em 2007, houve um grande interesse da comunidade científica nesses materiais, como mostrado na Fig. 1.3. Embora seja uma descoberta bastante recente, esses materiais apresentaram potenciais aplicações em spintrônica [22-25] e computação quântica topológica [26, 27] que foram responsáveis por um enorme interesse na teoria fundamental dos isolantes topológicos, assim como os sistemas físicos apresentados nessa tese.

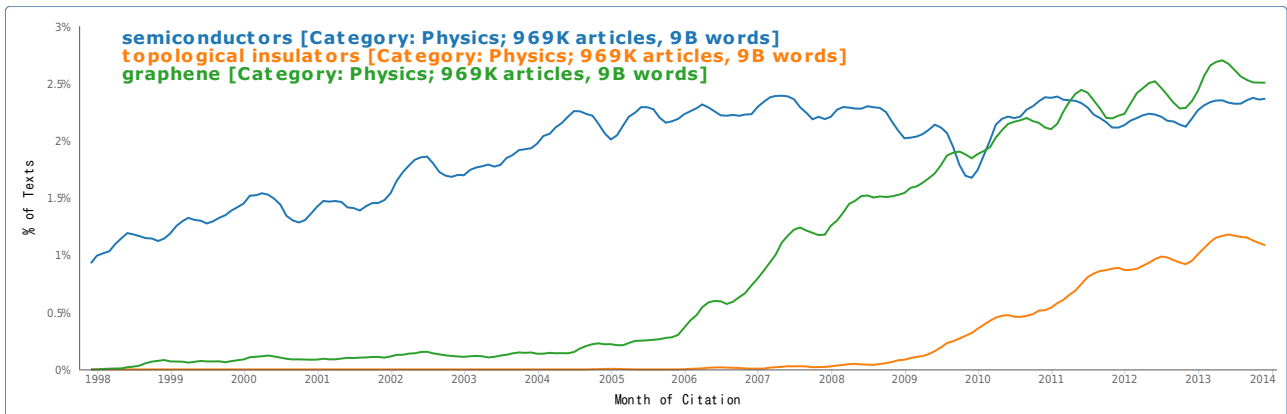

Figura 1.3: Porcentagens de artigos submetidos no arXiv.org com as palavras: "semiconductors" (em azul), "graphene" (em verde), e "topological insulators" (em laranja).

No Capítulo 2 vamos revisar os conceitos teóricos básicos da Física da Matéria Condensada para o estudo de topologia de bandas. Também vamos introduzir o conceito de invariante topológico $\mathbf{Z}_{2}$ para materiais em duas e três dimensões, com exemplos de materiais nessas classes.

No Capítulo 3 vamos apresentar os resultados do estudo de defeitos do tipo falhas de empilhamentos nos isolantes topológicos $\mathrm{Bi}_{2} \mathrm{Se}_{3}$ e $\mathrm{Bi}_{2} \mathrm{Te}_{3}$. As propriedades estruturais, energias de formações e propriedades eletrônicas dessas falhas de empilhamentos foram calculadas a partir de métodos de primeiros princípios baseados na Teoria do Funcional da Densidade (ver Apêndice A).

No Capítulo 4 iremos apresentar o estudo das interações dos estados metálicos de superfícies dos isolantes topológicos com as 
bandas de valência ou condução de um semicondutor. Nesse estudo é apresentado um Hamiltoniano efetivo para descrever essa interação que prevê o desdobramento do cone de Dirac na região da interface. O modelo é corroborado através de cálculos de primeiros princípios para a interface $\mathrm{Bi}_{2} \mathrm{Se}_{3} / \mathrm{GaAs}$. Além disso, também são apresentados cálculos de estabilidade de formação e estrutura eletrônica das superfícies de GaAs com tratamento de Se, que foram utilizadas no estudo das interfaces $\mathrm{Bi}_{2} \mathrm{Se}_{3} / \mathrm{GaAs}$ dos nossos cálculos de primeiros princípios.

No Capítulo 5 vamos apresentar cálculos de estabilidade de formação e de estrutura eletrônica de nanoestradas de germaneno imersas em germanano. O germaneno é um isolante topológico 2D que pode surgir da desidrogenação do germanano. É mostrado que através da dissociação de hidrogênio controlada, podemos construir nanoestruturas 1D (nanoestradas) com estados de bordas metálicos com polarização de spin. Esses estados metálicos podem originar correntes de spin sem dissipação com potenciais aplicações em spintrônica e nanoeletrônica.

No Capítulo 6 vamos apresentar o estudo da estabilidade de formação e das propriedades eletrônicas das ligas desordenadas hexagonais de $\mathrm{Si}_{x} \mathrm{Ge}_{1-x}$. É mostrado através do argumento de continuidade adiabática que as ligas desordenadas de $\mathrm{Si}_{x} \mathrm{Ge}_{1-x}$ também são isolantes topológicos 2D e podem apresentar efeitos Hall quântico de spin. Essa topologia de bandas também é apresentada através do argumento do comportamento em $W$ do gap de energia em função do campo elétrico externo aplicado. Por fim, é mostrado nesse capítulo que ligas ordenadas de SiGe e desordens não-SQSS (não-aleatórias) podem gerar propriedades eletrônicas diferentes dos isolantes topológicos 2D.

Finalmente no Capítulo 7 iremos apresentar as conclusões gerais do estudo das estruturas eletrônicas dos isolantes topológicos em duas e três dimensões. 


\section{Isolantes topológicos}

In these days the angel of topology and the devil of abstract algebra fight for the soul of every individual discipline of mathematics.

Hermann Weyl

\subsection{Introdução}

Entre os materiais mais estudados na Física da Matéria Condensada estão os cristais [2,28], formados por arranjos ordenados e periódicos de átomos, moléculas ou íons mantidos coesos através de ligações iônicas, covalentes, de higrodênio ou de van der Waals.

Para o estudo desses sistemas nessa tese vamos adotar a aproximação adiabática de Born-Oppenheimer, em que o operador de energia cinética dos núcleos é desprezado devido as diferenças de escalas de tempos de movimentos entre os núcleos e os elétrons. O Hamiltoniano do sistema pode ser desacoplado em um Hamiltoniano nuclear e um Hamiltoniano eletrônico. A partir deste desacoplamento, podemos determinar as propriedades físicas dos 
elétrons via diagonalizações do Hamiltoniano eletrônico e configurações estáticas dos íons.

Lançando mão da aproximação adiabática de Born-Oppenheimer, podemos escrever o Hamiltoniano eletrônico total do sistema como

$$
H=\sum_{\mathbf{R}} H(\mathbf{R}),
$$

onde $H(\mathbf{R})$ é o Hamiltoniano de uma célula unitária que se repete periodicamente pelo cristal, e $\mathbf{R}$ são os vetores da periodicidade do cristal, dados por

$$
\mathbf{R}=\sum_{i=1}^{D} n_{i} \mathbf{a}_{i},
$$

onde $n_{i}$ são números inteiros, $D$ é a dimensão da periodicidade do cristal, e $a_{i}$ são os vetores da base que caracterizam os cristais. $\mathrm{O}$ conjunto de pontos que podem ser localizados pelos vetores $\mathbf{R}$ é conhecido como rede de Bravais. Os vetores da base $\mathbf{a}_{1}$ e $\mathbf{a}_{2}$ da rede de Bravais de um cristal hexagonal de átomos em $2 D$ são mostrados na Fig. 2.1.

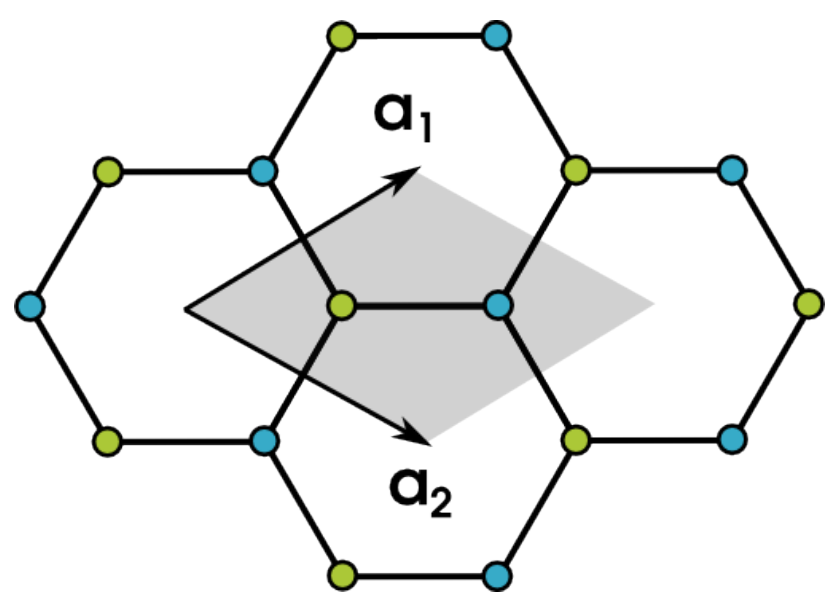

Figura 2.1: Vetores da base da rede de Bravais de um cristal hexagonal $2 D$. A área hachurada em cinza representa uma célula unitária possível para esta rede hexagonal. 
Para cada vetor $\mathbf{R}$ da rede de Bravais, é definida uma célula unitária com $m$ átomos, localizados por vetores $\boldsymbol{\delta}_{j}$, com $j=1, \ldots, m$. Esses vetores são conhecidos como vetores da base do cristal.

\subsection{Teoria de bandas}

\subsubsection{Hamiltoniano de Bloch}

Para Hamiltonianos periódicos como os encontrados nos cristais, podemos descrever o sistema completamente a partir de uma célula unitária que se repete ao longo do cristal. A diagonalização de um Hamiltoniano nessa célula unitária descreve todas as autoenergias e autoestados dos sólidos cristalinos, e possibilita a determinação de propriedades eletrônicas, magnéticas, ópticas e de transporte.

Para descrever as translações por vetores $\mathbf{R}$ da periodicidade do sistema, vamos definir o operador de translação $T_{\mathbf{R}}$, tal que

$$
T_{\mathbf{R}} H(\mathbf{r}) T_{\mathbf{R}}^{-1}=H(\mathbf{r}+\mathbf{R}),
$$

onde $H(\mathbf{r})$ é o Hamiltoniano eletrônico (na aproximação de campo médio) na posição r. Nos estados quânticos, o operador de translação atua da seguinte forma

$$
T_{\mathbf{R}}\left\langle\mathbf{r} \mid \psi_{n}\right\rangle=\left\langle\mathbf{r}+\mathbf{R} \mid \psi_{n}\right\rangle
$$

O grupo de simetria de translações dos sistemas cristalinos nos permite provar o seguinte teorema.

Teorema 1 (Bloch). Os autoestados de um Hamiltoniano periódico são dados por uma onda plana modulada por uma função com a mesma periodicidade do Hamiltoniano.

Prova. Para provar esse teorema é necessário notar que duas 
translações consecutivas fornecem

$$
T_{\mathbf{R}_{1}} T_{\mathbf{R}_{2}}\left\langle\mathbf{r} \mid \psi_{n}\right\rangle=\left\langle\mathbf{r}+\mathbf{R}_{1}+\mathbf{R}_{2} \mid \psi_{n}\right\rangle=T_{\mathbf{R}_{2}} T_{\mathbf{R}_{1}}\left\langle\mathbf{r} \mid \psi_{n}\right\rangle
$$

Da equação (2.5) podemos observar que o grupo de translações é um grupo abeliano. Como as representações irredutiveis dos grupos abelianos são unidimensionais [29], então

$$
T_{\mathbf{R}}\left\langle\mathbf{r} \mid \psi_{n}\right\rangle=\left\langle\mathbf{r}+\mathbf{R} \mid \psi_{n}\right\rangle=\lambda_{n}(\mathbf{R})\left\langle\mathbf{r} \mid \psi_{n}\right\rangle
$$

Multiplicando a equação (2.6) por seu complexo conjugado e integrando em todo o espaço, obtemos

$$
\left|\lambda_{n}(\mathbf{R})\right|^{2}=1
$$

ou seja,

$$
\lambda_{n}(\mathbf{R})=\mathrm{e}^{i \zeta_{n}(\mathbf{R})}
$$

onde $\zeta_{n}(\mathbf{R})$ é uma função real. Usando a parametrização da equação (2.8) no caso de duas translações consecutivas da equação (2.5), chegamos em

$$
\zeta_{n}\left(\mathbf{R}_{1}\right)+\zeta_{n}\left(\mathbf{R}_{2}\right)=\zeta_{n}\left(\mathbf{R}_{1}+\mathbf{R}_{2}\right)
$$

A equação (2.9) é uma condição de linearidade da função $\zeta_{n}$ dos vetores de translações $\{\mathbf{R}\}$ no espaço real. A forma mais geral das funções $\zeta_{n}(\mathbf{R})$ que satisfaz a equação (2.9) é da forma

$$
\zeta_{n}(\mathbf{R})=\mathbf{k} \cdot \mathbf{R}
$$

onde a arbitraridade das funções $\zeta_{n}$ são manifestadas pela arbitrariedade dos vetores $\mathrm{k}$ da equação $(2.10)$.

De fato, translações das funções de onda $\left\langle\mathbf{r} \mid \psi_{n}\right\rangle$ por vetores $\mathbf{R}$ 
fornecem

$$
\left\langle\mathbf{r}+\mathbf{R} \mid \psi_{n}\right\rangle=\mathrm{e}^{i \mathbf{k} \cdot \mathbf{R}}\left\langle\mathbf{r} \mid \psi_{n}\right\rangle
$$

Agora, definindo a função

$$
\left\langle\mathbf{r} \mid u_{n \mathbf{k}}\right\rangle=\mathrm{e}^{-i \mathbf{k} \cdot \mathbf{r}}\left\langle\mathbf{r} \mid \psi_{n}\right\rangle
$$

e substituindo na equação (2.11), encontramos

$$
\left\langle\mathbf{r} \mid u_{n \mathbf{k}}\right\rangle=\left\langle\mathbf{r}+\mathbf{R} \mid u_{n \mathbf{k}}\right\rangle
$$

que é uma condição de periodicidade para a função $\left\langle\mathbf{r} \mid u_{n \mathbf{k}}\right\rangle$. Multiplicando a equação (2.12) por $\mathrm{e}^{i \mathbf{k} \cdot \mathbf{r}}$, finalmente obtemos

$$
\left\langle\mathbf{r} \mid \psi_{n}\right\rangle=\mathrm{e}^{i \mathbf{k} \cdot \mathbf{r}}\left\langle\mathbf{r} \mid u_{n \mathbf{k}}\right\rangle,
$$

que é a prova do teorema de Bloch.

Os estados $\left|u_{n \mathbf{k}}\right\rangle$, conhecidos como estados de Bloch, são autoestados do seguinte Hamiltoniano

$$
\mathcal{H}(\mathbf{k})=\mathrm{e}^{i \mathbf{k} \cdot \mathbf{r}} H \mathrm{e}^{-i \mathbf{k} \cdot \mathbf{r}},
$$

que é chamado de Hamiltoniano de Bloch. Esse Hamiltoniano possui os mesmos autovalores do Hamiltoniano eletrônico $H$.

\subsubsection{Rede reciproca e zona de Brillouin}

O Hamiltoniano de Bloch apresenta propriedades de simetria por translações discretas da seguinte forma

$$
\mathcal{H}(\mathbf{k}+\mathbf{G})=\mathcal{H}(\mathbf{k})
$$


onde os vetores G são dados por

$$
\mathbf{G}=\sum_{i=1}^{D} m_{i} \mathbf{b}_{i}
$$

com $m_{i}$ inteiros, e vetores da base $\mathbf{b}_{i}$ determinados pelo sistema. $\mathrm{O}$ vetor $\mathbf{b}_{i}$ é obtido da relação

$$
\mathbf{a}_{i} \cdot \mathbf{b}_{j}=2 \pi \delta_{i j}
$$

onde $\delta_{i j}$ é o delta de Kronecker.

A rede formada pelos vetores $\mathrm{G}$, dados na equação (2.17) é chamada de rede reciproca, que também é uma rede de Bravais como os vetores da equação (2.2).

A partir dos vetores da base $\mathbf{b}_{i}, \operatorname{com} i=1, \ldots, D$, podemos criar uma célula unitária no espaço- $k$ que pode ser vista como um domínio fundamental para o Hamiltoniano de Bloch, isto é, o Hamiltoniano definido nestes pontos descreve completamente o sistema. Um procedimento para criar esta célula unitária de forma que respeite todas as simetrias do material é através da condição

$$
|\mathbf{k}| \leq|\mathbf{k}-\mathbf{G}|
$$

para qualquer vetor $\mathrm{G}$ da rede reciproca. Os vetores que satisfazem a equação (2.19) formam a zona de Brillouin (BZ, acrônimo do termo em inglês Brillouin Zone). Os vetores da rede recíproca e a zona de Brillouin de uma rede hexagonal $2 D$ são mostrados na Fig. 2.2

Devido a condição de periodicidade (2.16), podemos dizer que há identificações de pontos $\mathrm{k}$ com $\mathrm{k}+\mathrm{G}$, que tornam as zonas de Brillouin em $D$ dimensões topologicamente equivalentes ${ }^{1}$ à um toro $T^{D}$. Uma ilustração esquemática para o caso $D=2$ dessa

\footnotetext{
${ }^{1}$ Duas superfícies $A$ e $B$ são topologicamente equivalentes se existe uma transformação contínua (ou deformação) que leva a superfície $A$ em $B$.
} 


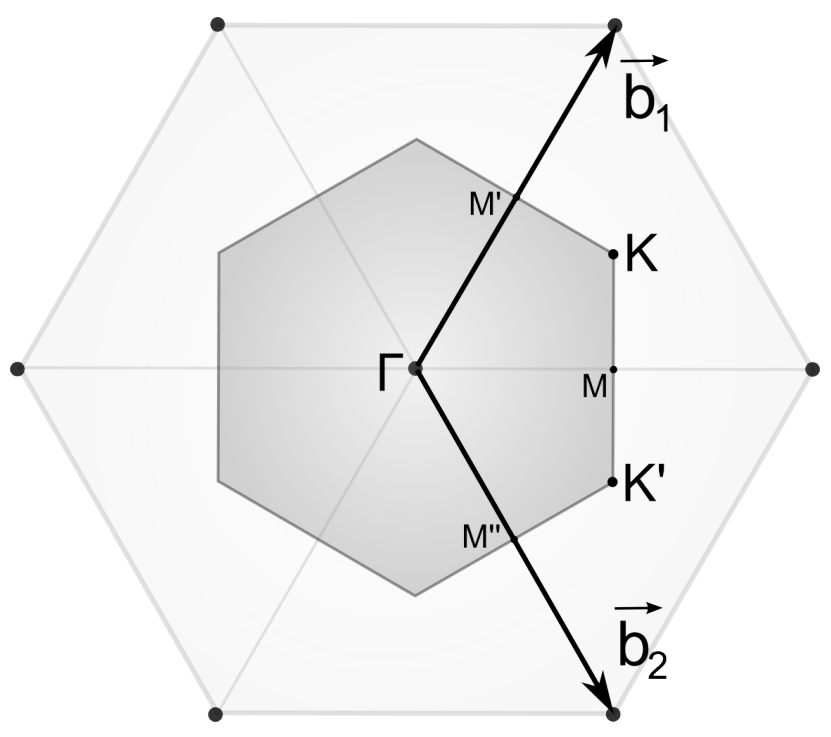

Figura 2.2: Vetores da base da rede recíproca e zona de Brillouin de uma rede hexagonal $2 D$.

equivalência topológica é mostrada na Fig. 2.3.
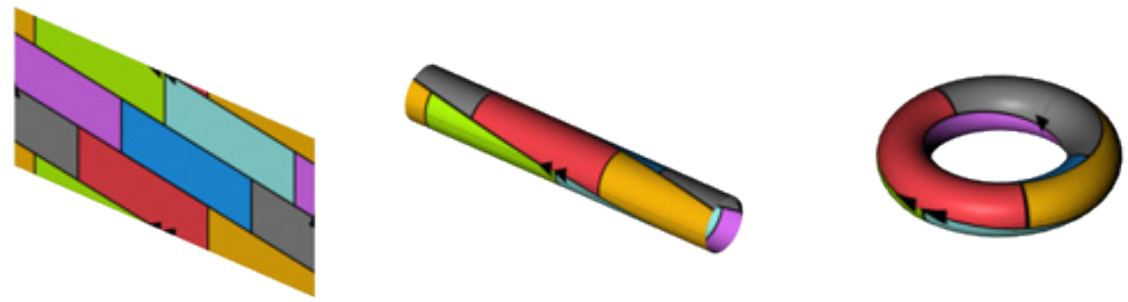

Figura 2.3: Representação esquemática da equivalência topológica entre um conjunto com condições periódicas de contorno e um toro $T^{2}$.

\subsubsection{Estrutura de bandas}

Uma vez determinado o Hamiltoniano de Bloch $\mathcal{H}(\mathbf{k})$, podemos obter os estados de Bloch através da diagonalização da seguinte 
equação

$$
\mathcal{H}(\mathbf{k})\left|u_{n \mathbf{k}}\right\rangle=\epsilon_{n}(\mathbf{k})\left|u_{n \mathbf{k}}\right\rangle
$$

Os autovalores $\epsilon_{n}(\mathbf{k})$ do Hamiltoniano de Bloch são chamados de bandas de energias, enquanto que o conjunto de todos os autovalores (espectro) de um material é chamado de estrutura de bandas. Essas bandas podem ser representadas graficamente através de caminhos na zona de Brillouin como mostrado na Fig. 2.4(a). O caminho percorrido e os pontos de alta simetria $(\Gamma, X, W, K, L, U)$ são mostrados na Fig. 2.4(b).
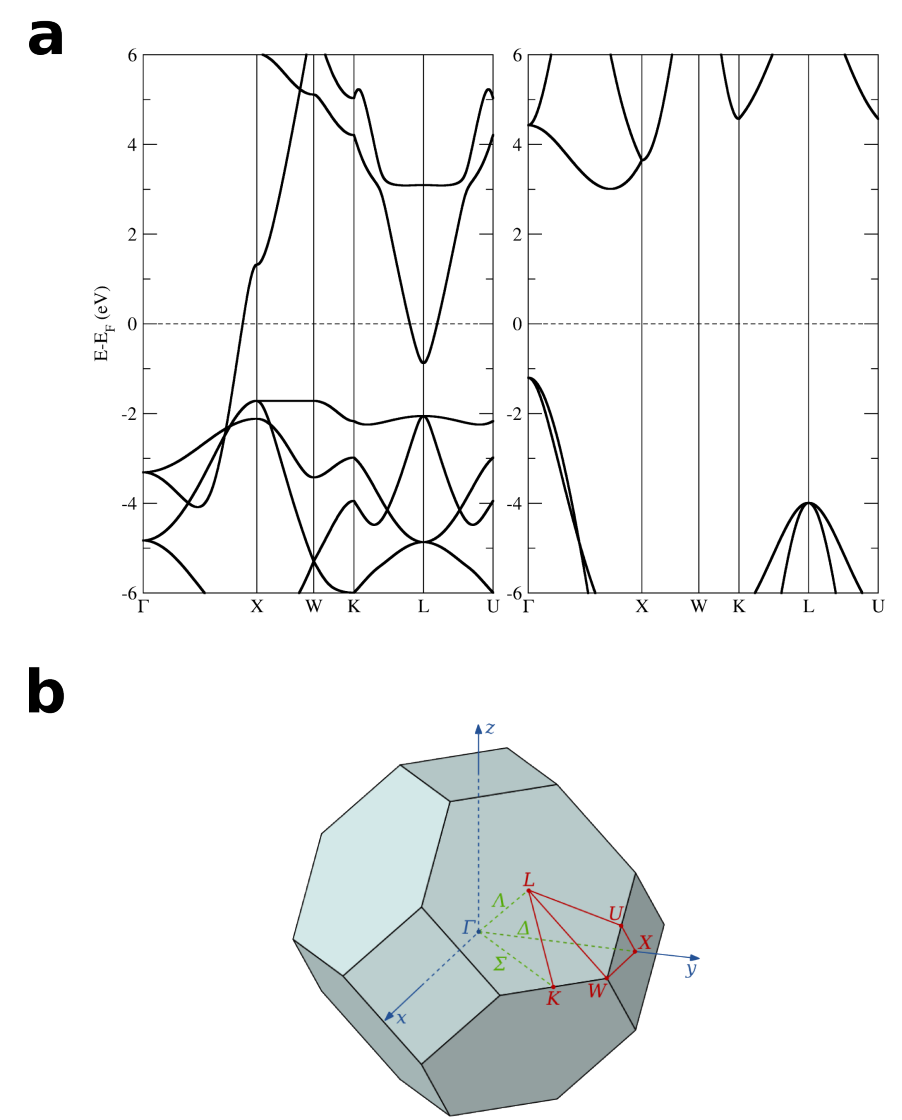

Figura 2.4: (a) Estruturas de bandas do cristal $f c c$ de $\mathrm{Au}$, e do diamante. (b) Zona de Brillouin de uma rede $f c c$ e seus pontos de alta simetria. 


\subsection{Teoria de Berry}

\subsubsection{Transporte paralelo}

Vamos considerar um estado de Bloch $\left|u_{n \mathbf{k}}\right\rangle$ que é transportado para um estado na sua vizinhança da seguinte forma

$$
\left|u_{n \mathbf{k}}\right\rangle \longrightarrow\left|u_{n \mathbf{k}+d \mathbf{k}}\right\rangle=\left|u_{n \mathbf{k}}\right\rangle+\nabla_{\mathbf{k}}\left|u_{n \mathbf{k}}\right\rangle \cdot d \mathbf{k}
$$

Nesse transporte de estados, há componentes paralelas e perpendiculares ao estado inicial $\left|u_{n \mathbf{k}}\right\rangle$. As componentes perpendiculares possuem projeções nulas no estado inicial e não devem contribuir para as características topológicas dos estados de Bloch que serão calculadas. As componentes paralelas são obtidas a partir da aplicação do projetor $\mathcal{P}_{\|}=\left|u_{n \mathbf{k}}\right\rangle\left\langle u_{n \mathbf{k}}\right|$ na equação (2.21), fornecendo

$$
\left|u_{n \mathbf{k}}\right\rangle \longrightarrow\left|u_{n \mathbf{k}}\right\rangle\left\langle u_{n \mathbf{k}} \mid u_{n \mathbf{k}+d \mathbf{k}}\right\rangle=\left|u_{n \mathbf{k}}\right\rangle+\left|u_{n \mathbf{k}}\right\rangle\left\langle u_{n \mathbf{k}}\left|\nabla_{\mathbf{k}}\right| u_{n \mathbf{k}}\right\rangle \cdot d \mathbf{k}
$$

As variações $\left|u_{n \mathbf{k}}\right\rangle\left\langle u_{n \mathbf{k}}\left|\nabla_{\mathbf{k}}\right| u_{n \mathbf{k}}\right\rangle \cdot d \mathbf{k}$ dadas ao estado inicial $\left|u_{n \mathbf{k}}\right\rangle$ são chamadas de transporte paralelo, e eles formam a base de alguns conceitos fundamentais geométricos e topológicos.

\subsubsection{Conexão e curvatura de Berry}

O transporte paralelo de estados de Bloch também pode ser escrito como

$$
\left|u_{n \mathbf{k}}\right\rangle \longrightarrow\left(1+i \mathbf{A}_{n} \cdot d \mathbf{k}\right)\left|u_{n \mathbf{k}}\right\rangle
$$

onde

$$
\mathbf{A}_{n}(\mathbf{k})=-i\left\langle u_{n \mathbf{k}}\left|\nabla_{\mathbf{k}}\right| u_{n \mathbf{k}}\right\rangle
$$

é a conexão de Berry.

O transporte paralelo também pode ser feito ao longo de um 
caminho, de comprimento finito, através de uma integral de linha da conexão de Berry. O resultado do transporte paralelo partindo de um estado inicial $\left|u_{n \mathbf{k}_{i}}\right\rangle$ e chegando em um estado final $\left|u_{n \mathbf{k}_{f}}\right\rangle$ é indicado por

$$
\left\langle u_{n \mathbf{k}_{f}} \mid u_{n \mathbf{k}_{i}}\right\rangle=\int_{\mathbf{k}_{i}}^{\mathbf{k}_{f}} d \mathbf{k} \cdot \mathbf{A}_{n}(\mathbf{k}) .
$$

Se o percurso da integração for um caminho fechado $\mathscr{C}\left(\mathbf{k}_{i}=\mathbf{k}_{f}\right)$, então os estados iniciais e finais, denotados agora por $\left|u_{n \mathbf{k}}^{\text {inicial }}\right\rangle$ e $\left|u_{n \mathrm{k}}^{\text {final }}\right\rangle$ vão mudar pela fase

$$
\gamma_{n}=\left\langle u_{n \mathbf{k}}^{\text {final }} \mid u_{n \mathbf{k}}^{\text {inicial }}\right\rangle=\oint_{\mathscr{C}} d \mathbf{k} \cdot \mathbf{A}_{n}(\mathbf{k}) .
$$

A integração da conexão de Berry ao longo de um caminho fechado é conhecido como fase de Berry (ou fase geométrica) [30,31]. Esta fase não depende da evolução dinâmica dos estados de Bloch, mas somente do espaço de parâmetros em que os estados variam. No caso dos sólidos cristalinos, o espaço de parâmetros é a zona de Brillouin [32].

Embora a fase de Berry seja um conceito quântico e geométrico, podemos ver sua interpretação física através de análogos clássicos. Na teoria clássica, o conceito análogo à fase de Berry é o ângulo de Hannay. Essa grandeza é uma medida de quanto um vetor tangente à uma superfície curva muda ao percorrer um caminho fechado nesta superfície.

Considere a superfície curva como uma esfera, e um vetor tangente inicial no equador desta superfície [ver Fig. (2.5)]. Se o vetor tangente for paralelamente transportado do ponto A para o polo norte $\mathrm{N}$ através de uma geodésica ${ }^{2}$, seguido por outro transporte paralelo do polo norte $\mathrm{N}$ ao equador para o ponto $\mathrm{B}$, e de um terceiro transporte paralelo realizado do ponto $B$ para o ponto A ao longo do equador. O vetor tangente final é uma rotação de $\frac{\pi}{2}$ do

\footnotetext{
${ }^{2}$ Curvas obtidas pelas minimizações das distâncias.
} 
vetor tangente inicial. Essa variação angular de $\frac{\pi}{2}$ é o ângulo de Hannay do transporte paralelo total descrito acima. Esse transporte paralelo e o ângulo de Hannay $\alpha$ são ilustrados na Fig. (2.5).

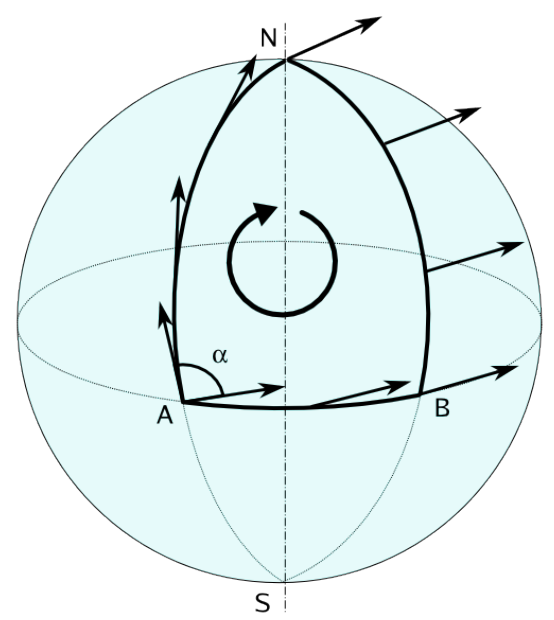

Figura 2.5: Transporte paralelo de um vetor ao longo de um caminho fechado sobre uma esfera. O transporte é realizado através dos caminhos de A para N, de N para B, e de B para A. O ângulo de rotação $\alpha$ entre o estado inicial e o estado final do vetor é chamado de holonomia da conexão de Berry.

Do ponto de vista da geometria diferencial, a medida da mudança de um vetor tangente em uma superfície curva ao longo de um caminho fechado é conhecido como holonomia. Nesse sentido, a fase de Berry pode ser vista como a holonomia da conexão de Berry adquirida ao longo de um processo adiabático e cíclico [33].

Já que a fase de Berry é obtida através de uma integração de um campo vetorial (conexão de Berry) ao longo de um caminho fechado, é possível utilizar o teorema de Stokes para transformar esta integração de linha em uma integração de superfície. Assim, a fase de Berry será

$$
\gamma_{n}(\mathscr{S})=\int_{\mathscr{S}} d^{2} \mathbf{k} \cdot \mathbf{B}_{n}(\mathbf{k}),
$$


onde

$$
\mathbf{B}_{n}(\mathbf{k})=\nabla_{\mathbf{k}} \times \mathbf{A}_{n}(\mathbf{k})
$$

é a curvatura de Berry, e $\mathscr{S}$ é a superfície de integração no espaço$k$.

\subsection{Simetria de reversão temporal}

A operação de simetria para sólidos cristalinos é efetuada através do operador

$$
\mathcal{T}: \mathbf{r} \rightarrow \mathbf{r}, \quad \mathcal{T}: \mathbf{k} \rightarrow-\mathbf{k},
$$

onde $\mathbf{r}$ são vetores posições no espaço e $\mathbf{k}$ são vetores na zona de Brillouin. No espaço de Hilbert, o operador de reversão temporal (denotado por $\Theta$ neste espaço) atua da seguinte forma

$$
\Theta \mathcal{H}(\mathbf{k}) \Theta^{-1}=\mathcal{H}(-\mathbf{k})
$$

onde $\mathcal{H}(\mathbf{k})$ são os Hamiltonianos de Bloch. O operador $\Theta$ é um operador anti-linear [34] que pode ser representado como

$$
\Theta=U K
$$

onde $U=-i s_{y}$ é um operador unitário e $K$ é o operador de conjugação complexa. Esse operador de revesão temporal obedece a condição

$$
\Theta^{2}=-1
$$

como visto na referência [34].

Embora um sistema com reversão temporal satisfaça a condição (2.30) em todos os pontos $k$ da zona de Brillouin, há alguns pontos específicos denotados por $\Gamma_{\alpha}, \operatorname{com} \alpha=1, \ldots, \mathcal{N}$, em que o 
Hamiltoniano comuta com o operador $\Theta$, isto é,

$$
\left[\Theta, \mathcal{H}\left(\Gamma_{\alpha}\right)\right]=0
$$

resultando em

$$
\mathcal{H}\left(\Gamma_{\alpha}\right)=\mathcal{H}\left(-\Gamma_{\alpha}\right)
$$

Os pontos em que o Hamiltoniano comuta com o operador $\Theta$ são chamados de TRIM (acrônimo do termo em inglês Time-reversal invariant momenta). O número inteiro $\mathcal{N}$ é a quantidade de TRIM que existem na zona de Brillouin.

As coordenadas dos TRIM são dadas por

$$
\Gamma_{\alpha}=\frac{1}{2} \sum_{i=1}^{D} n_{i} \mathbf{b}_{i}, \quad n_{i}=0,1
$$

Dessa forma, o número de TRIM será $\mathcal{N}=2^{D}$, onde $D$ é a dimensão da zona de Brillouin. Os TRIM das zonas de Brillouin quadrada e hexagonal podem ser vistos na Fig. 2.6.

Podemos definir o operador de reversão temporal na base de estados de Bloch da seguinte forma

$$
w_{i j}(\mathbf{k})=\left\langle u_{j,-\mathbf{k}}|\Theta| u_{i, \mathbf{k}}\right\rangle,
$$

onde $i$ e $j$ são os índices de bandas. Esses elementos de matrizes estão relacionados com o estado de Bloch da seguinde forma

$$
\left|u_{i,-\mathbf{k}}\right\rangle=\sum_{j} w_{i j}^{*}(\mathbf{k}) \Theta\left|u_{j, \mathbf{k}}\right\rangle .
$$

Podemos notar que a matriz $\mathbf{w}(\mathbf{k})$ é unitária, i.e.,

$$
\sum_{i} w_{i l}^{*}(\mathbf{k}) w_{i j}(\mathbf{k})=\delta_{j l}
$$




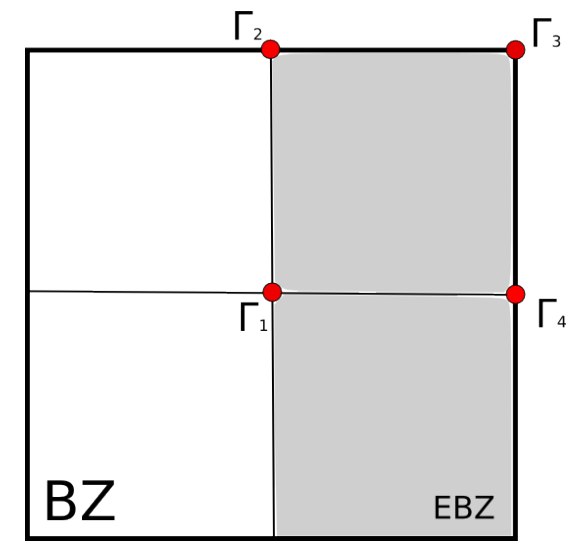

(a) Rede quadrada

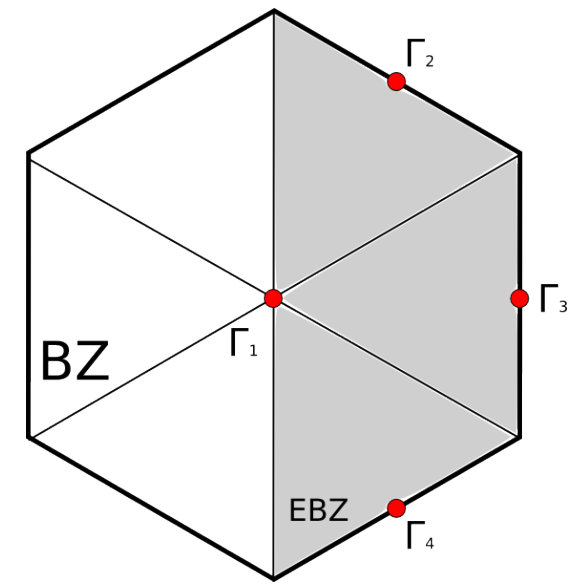

(b) Rede hexagonal

Figura 2.6: Zona de Brillouin Efetivas das redes 2D: (a) quadrada, (b) hexagonal. Os TRIM $\Gamma_{\alpha}$ nessas zonas de Brillouin são mostrados em vermelho.

e obedece a seguinte propriedade

$$
w_{j i}(\mathbf{k})=-w_{i j}(-\mathbf{k}) .
$$

Nos pontos TRIM a matriz $w$ é

$$
w_{i j}\left(\Gamma_{\alpha}\right)=-w_{j i}\left(\Gamma_{\alpha}\right),
$$

ou seja, torna-se antissimétrica.

Para o caso em que temos apenas duas bandas, que são relacionadas pela simetria de reversão temporal, a matriz $w_{i j}(\mathbf{k})$ torna-se

$$
w\left(\Gamma_{\alpha}\right)=\left(\begin{array}{cc}
0 & w_{12}\left(\Gamma_{\alpha}\right) \\
-w_{12}\left(\Gamma_{\alpha}\right) & 0
\end{array}\right) .
$$

A conexão de Berry para esse modelo de duas bandas é dada pela conexão de Berry não-abeliana

$$
\mathbf{A}_{i j}(\mathbf{k})=-i\left\langle u_{i \mathbf{k}}\left|\nabla_{\mathbf{k}}\right| u_{j \mathbf{k}}\right\rangle .
$$


Usando as propriedades do operador de reversão temporal, podemos encontrar que

$$
\mathbf{A}(-\mathbf{k})=\mathbf{w}(\mathbf{k}) \mathbf{A}^{*}(\mathbf{k}) \mathbf{w}^{\dagger}(\mathbf{k})+i \mathbf{w}(\mathbf{k}) \boldsymbol{\nabla}_{\mathbf{k}} \mathbf{w}^{\dagger}(\mathbf{k})
$$

Tomando o traço e substituindo $\mathrm{k}$ por $-\mathbf{k}$, obtemos

$$
\operatorname{tr}[\mathbf{A}(\mathbf{k})]=\operatorname{tr}[\mathbf{A}(-\mathbf{k})]+i \operatorname{tr}\left[\mathbf{w}^{\dagger}(\mathbf{k}) \boldsymbol{\nabla}_{\mathbf{k}} \mathbf{w}(\mathbf{k})\right]
$$

A equação (2.44) será utilizada na seção seguinte para o cálculo dos invariantes topológicos para sistemas com simetria de reversão temporal.

\subsection{Topologia de bandas $Z_{2}$}

A partir do modelo de duas bandas com simetria de reversão temporal, podemos calcular invariantes topológicos associados aos estados de Bloch como realizado na topologia de bandas $\mathbf{Z}_{2}$ de $\mathrm{Fu}$ e Kane [35]. Para calcular esses invariantes topológicos vamos considerar um sistema unidimensional (1D) com constante de rede $a$. Os dois estados de Bloch desse modelo unidimensional são $\left|u_{1}(k)\right\rangle$ e $\left|u_{2}(k)\right\rangle$. Considerando que o Hamiltoniano de Bloch também é periódico no tempo, com período $T$, obtemos as seguintes propriedades

$$
H(t+T)=H(t)
$$

e

$$
H(-t)=\Theta H(t) \Theta^{-1}
$$

Da teoria moderna de ferroeletricidade [36] podemos calcular a polarização de carga pela seguinte integração da conexão de Berry

$$
P_{\rho}=\int_{-\pi}^{+\pi} \frac{d k}{2 \pi} \operatorname{tr}[\mathbf{A}(k)]
$$


e também a polarização de reversão temporal

$$
P_{\theta}=P_{1}-P_{2}
$$

onde

$$
P_{i}=\int_{-\pi}^{+\pi} \frac{d k}{2 \pi} A_{i i}(k)
$$

O Hamiltoniano que satisfaz as equações (2.45) e (2.46) é invariante por reversões temporais em $t=0$ e $t=T / 2$. Nesses instantes o Hamiltoniano é duplamente degenerado para qualquer ponto $k$, com estados degenerados $\left|u_{1}(k)\right\rangle$ e $\left|u_{2}(k)\right\rangle=\Theta\left|u_{1}(-k)\right\rangle$, exceto por uma fase local. Nesses instantes temos que

$$
\Theta\left|u_{1}(k)\right\rangle=-\mathrm{e}^{-i \chi(-k)}\left|u_{2}(-k)\right\rangle
$$

e

$$
\Theta\left|u_{2}(k)\right\rangle=\mathrm{e}^{i \chi(-k)}\left|u_{1}(-k)\right\rangle
$$

Com essas relações podemos calcular a matriz $\mathbf{w}$ de reversão temporal,

$$
w(k)=\left(\begin{array}{cc}
0 & \mathrm{e}^{-i \chi(k)} \\
-\mathrm{e}^{-i \chi(-k)} & 0
\end{array}\right) .
$$

Calculando $P_{1}$ nos instantes $t=0$ e $t=T / 2$, chegamos em

$$
\begin{aligned}
P_{1} & =\frac{1}{2 \pi} \int_{0}^{\pi} d k A_{11}(k)+\frac{1}{2 \pi} \int_{-\pi}^{0} d k A_{11}(k) \\
& =\frac{1}{2 \pi}\left[\int_{0}^{\pi} d k A_{11}(k)+A_{11}(-k)\right] \\
& =\frac{1}{2 \pi} \int_{0}^{\pi} d k\left[A_{11}(k)+A_{22}(k)-\frac{\partial}{\partial k} \chi(k)\right] \\
& =\int_{0}^{\pi} \frac{d k}{2 \pi} \operatorname{tr}[\mathbf{A}(k)]-\frac{1}{2 \pi}[\chi(\pi)-\chi(0)] .
\end{aligned}
$$

Invertendo a equação $w_{12}(k)=\mathrm{e}^{-i \chi(k)}$, obtemos

$$
\chi(k)=i \log \left[w_{12}(k)\right]
$$


Substituindo a equação (2.54) na equação (2.53), chegamos em

$$
P_{1}=\frac{1}{2 \pi} \int_{0}^{\pi} \frac{d k}{2 \pi} \operatorname{tr}[\mathbf{A}(k)]-\frac{i}{2 \pi} \log \left[\frac{w_{12}(\pi)}{w_{12}(0)}\right] .
$$

Calculando a quantidade $P_{2}$ e substituindo na expressão para a polarização de reversão temporal, obtemos

$$
P_{\theta}=\frac{1}{i \pi} \log \left[\frac{\sqrt{w_{12}(0)^{2}}}{w_{12}(0)} \cdot \frac{w_{12}(\pi)}{\sqrt{w_{12}(\pi)^{2}}}\right]
$$

$\mathrm{O}$ argumento desse logaritmo pode ser +1 ou -1 . Já que $\log (-1)=$ $i \pi$, então $P_{\theta}$ pode ser 0 ou 1 .

Agora, definindo a diferença entre a polarização de reversão temporal $P_{\theta}$ entre os instantes 0 e $T / 2$ da forma

$$
\nu=P_{\theta}(T / 2)-P_{\theta}(0),
$$

podemos medir o quanto um estado de Bloch muda de polaridade de reversão temporal. Essa diferença $\nu$ é definida de modo que

$$
(-1)^{\nu}=\prod_{\alpha=1}^{4} \frac{w_{12}\left(\Gamma_{\alpha}\right)}{\sqrt{w_{12}\left(\Gamma_{\alpha}\right)^{2}}},
$$

onde $\Gamma_{\alpha}$ são os “TRIM" considerando a periodicidade em $t$.

De forma geral, para um sistema de $N$ bandas temos que

$$
\begin{gathered}
\Theta\left|u_{2}^{n}(k)\right\rangle=\mathrm{e}^{-i \chi_{n}(k)}\left|u_{1}^{n}(-k)\right\rangle, \\
\Theta\left|u_{1}^{n}(k)\right\rangle=-\mathrm{e}^{-i \chi_{n}(-k)}\left|u_{2}^{n}(-k)\right\rangle,
\end{gathered}
$$

e a polarização de reversão temporal será dada por

$$
P_{\theta}=\frac{1}{i \pi}\left[\frac{\sqrt{\operatorname{det}[w(0)]}}{\operatorname{Pf}[w(0)]} \cdot \frac{\operatorname{Pf}[w(\pi)]}{\sqrt{\operatorname{det}[w(\pi)]}}\right] \text {, }
$$


onde o Pfaffiano da matriz $w$ é dado por

$$
\operatorname{Pf}\left[w\left(\Gamma_{\alpha}\right)\right]=w_{12}\left(\Gamma_{\alpha}\right) w_{34}\left(\Gamma_{\alpha}\right) \ldots w_{2 N-1,2 N}\left(\Gamma_{\alpha}\right)
$$

$\mathrm{e}$

$$
\operatorname{Pf}[w]^{2}=\operatorname{det}[w] .
$$

Usando as propriedades do Pfaffiano, podemos chegar finalmente na equação

$$
(-1)^{\nu}=\prod_{\alpha=1}^{4} \frac{\operatorname{Pf}\left[w\left(\Gamma_{\alpha}\right)\right]}{\sqrt{\operatorname{det}\left[w\left(\Gamma_{\alpha}\right)\right.}}
$$

No sistema 2D com espaço-k dado por pontos $\left(k_{x}, k_{y}\right)$ ao invés do espaço de fase $(t, k)$, a quantidade $\nu$ é um invariante topológico que define a topologia de bandas de um material. Se $\nu=1$ o material é um isolante topológico, e se $\nu=0$ o material é um isolante trivial (ou isolante ordinário).

Uma das principais consequências da topologia de bandas nãotrivial é a proteção topológica dos estados de superfícies. Devido a diferença de topologias de bandas entre dois meios, os estados de superfícies são robustos por perturbações que mantenham a simetria de reversão temporal. Em outras palavras, mantendo a simetria de reversão temporal, mesmos grandes perturbações no Hamiltoniano do isolante topológico não podem destruir os estados metálicos das superfícies. Devido à essa propriedade, os estados de superfícies também são chamados de estados topologicamente protegidos.

\subsubsection{Invariante $Z_{2}$ para materiais centrossimétri- $\cos$}

O cálculo do invariante topológico que define a topologia de bandas de um material é simplificada para materiais com simetria de 
inversão, além da simetria de reversão temporal. Definindo o operador de inversão como

$$
\Pi|\mathbf{r}, \sigma\rangle=|-\mathbf{r}, \sigma\rangle,
$$

onde $\mathbf{r}$ é a coordenada espacial e $\sigma$ é o spin, podemos notar que

$$
\Pi^{2}=1
$$

que significa que os autovalores de $\Pi$ são \pm 1 .

Nos pontos TRIM podemos notar que

$$
\Pi H \Pi^{-1}=H,
$$

quando usamos que $\Pi|\mathbf{k}, \sigma\rangle=|-\mathbf{k}, \sigma\rangle$. Os autoestados de Bloch nos TRIM são também autoestados do operador de inversão, e assim tem paridade bem definida.

Calculando a conexão e a curvatura de Berry não-abeliana para o sistema com simetria de inversão, notamos que

$$
\mathbf{B}_{n}(\mathbf{k})=\nabla_{\mathbf{k}} \times \mathbf{A}_{n}(\mathbf{k})
$$

satisfaz a condição

$$
\mathbf{B}_{n}(-\mathbf{k})=-\mathbf{B}_{n}(\mathbf{k})
$$

pela simetria de reversão temporal, e

$$
\mathbf{B}_{n}(-\mathbf{k})=+\mathbf{B}_{n}(\mathbf{k})
$$

pela simetria de inversão. Dessa forma, a única curvatura de Berry possivel para esse sistema é $\mathbf{B}_{n}(\mathbf{k})=0$ para qualquer $\mathbf{k} \in B Z$. Isso é possivel fazendo $\mathbf{A}_{n}(\mathbf{k})=0$. Para obter isso, vamos considerar o elemento de matriz

$$
\nu_{i j}(\mathbf{k})=\left\langle u_{i \mathbf{k}}|\Pi \Theta| u_{j \mathbf{k}}\right\rangle .
$$

A matriz $\nu$ é antissimétrica $\left(\nu_{i j}=-\nu_{j i}\right)$ e unitária $\left(\sum_{j} \nu_{i j}(\mathbf{k}) \nu_{l j}(\mathbf{k})^{*}=\right.$ 
$\left.\delta_{i l}\right)$. A relação entre a matriz $\nu$ e a conexão de Berry não-abeliana é

$$
\frac{i}{2} \operatorname{tr}\left[\nu^{\dagger} \nabla_{\mathbf{k}} \nu\right]=\mathbf{A}(\mathbf{k})
$$

Através das propriedades de unitariedades e do traço, podemos obter que

$$
\mathbf{A}(\mathbf{k})=\frac{i}{2} \boldsymbol{\nabla}_{\mathbf{k}} \log [\operatorname{det}(\nu(\mathbf{k}))]=i \boldsymbol{\nabla}_{\mathbf{k}} \log \operatorname{Pf}[\nu(\mathbf{k})] .
$$

Para termos $\mathbf{A}(\mathbf{k})=0$, temos que ter $\operatorname{Pf}[\nu(\mathbf{k})]=1$.

Para calcular os elementos de matrizes da matriz $w$, vamos primeiro considerar que os autovalores do operador П são dados por

$$
\Pi\left|u_{i}\left(\Gamma_{\alpha}\right)\right\rangle=\xi_{2 i}\left(\Gamma_{\alpha}\right)\left|u_{i}\left(\Gamma_{\alpha}\right)\right\rangle
$$

Dessa forma,

$$
\begin{aligned}
w_{i j}\left(\Gamma_{\alpha}\right) & =\left\langle u_{i}\left(-\Gamma_{\alpha}\right)|\Pi \Pi \Theta| u_{j}\left(\Gamma_{\alpha}\right)\right\rangle \\
& =\xi_{i}\left(\Gamma_{\alpha}\right)\left\langle u_{i}\left(\Gamma_{\alpha}\right)|\Pi \Theta| u_{j}\left(\Gamma_{\alpha}\right)\right\rangle \\
& =\xi_{i}\left(\Gamma_{\alpha}\right) \nu_{i j}\left(\Gamma_{\alpha}\right) .
\end{aligned}
$$

Para as bandas que são relacionadas pela simetria de reversão temporal (pares de Kramers), temos que $w_{i j}=-w_{j i} \neq 0$. Assim, $\xi_{i}=\xi_{j}=\xi_{2 n}$, onde $n$ é o $n$-ésimo par de Kramers no ponto $\Gamma_{\alpha}$. Dessa forma, temos que

$$
\operatorname{Pf}\left[w\left(\Gamma_{\alpha}\right)\right]=\operatorname{Pf}\left[\nu\left(\Gamma_{\alpha}\right)\right] \prod_{n=1}^{N} \xi_{2 n}\left(\Gamma_{\alpha}\right) .
$$

Já que $\operatorname{Pf}\left[\nu\left(\Gamma_{\alpha}\right)\right]=1$, nós obtemos

$$
\delta\left(\Gamma_{\alpha}\right)=\frac{\operatorname{Pf}\left[w\left(\Gamma_{\alpha}\right)\right]}{\sqrt{\operatorname{det}\left[w\left(\Gamma_{\alpha}\right)\right]}}=\prod_{n=1}^{N} \xi_{2 n}\left(\Gamma_{\alpha}\right) .
$$

Finalmente podemos notar que os invariantes topológicos $\mathbf{Z}_{2} \operatorname{com}$ 
simetria de inversão podem ser calculados simplesmente a partir das paridades dos estados de Bloch nos TRIM da seguinte forma

$$
(-1)^{\nu}=\prod_{\alpha=1}^{4} \delta\left(\Gamma_{\alpha}\right)=\prod_{\alpha=1}^{4} \prod_{n=1}^{\mathrm{N}} \xi_{2 n}\left(\Gamma_{\alpha}\right) .
$$

Essas paridades podem ser calculadas a partir de métodos de primeiros princípios baseados na Teoria do Funcional da Densidade (ver apêndice $\mathrm{A}$ ).

No apêndice C são mostradas as expressões para calcular os invariantes $\mathbf{Z}_{2}$ com ou sem simetria de inversão.

\subsubsection{Invariantes $\mathrm{Z}_{2}$ em três dimensões}

A partir de argumentos de homotopia, Moore e Balents [15] mostraram que há quatro invariantes $\mathbf{Z}_{2}$ para materiais em três dimensões. Para entender a construção dos quatro invariantes topológi$\cos \mathrm{Z}_{2}$ dos materiais $3 \mathrm{D}$ vamos considerar seis planos que contém quatro TRIM que originam um invariante $\mathbf{Z}_{2}$, como nos materiais 2D. Os seis planos para cálculos dos invariantes $\mathbf{Z}_{2}$ são mostrados na Fig. 2.7 e rotulados de $x_{0}, x_{1}, y_{0}, y_{1}, z_{0}$, e $z_{1}$. Para cada um desses planos podemos calcular um invariante $\mathbf{Z}_{2}$ como na equação (2.64). Esses invariantes $\mathbf{Z}_{2}$ por planos podem ser calculados para casos em que não há simetria de inversão, ou como na equação (2.78) para casos em que há simetria de inversão (centrossimétricos).

Entretanto, podemos notar que os produtos de invariantes topológicos $x_{0} x_{1}, y_{0} y_{1}$, e $z_{0} z_{1}$ fornecem o mesmo resultado, que é o produto dos Pfaffianos em todos os oito pontos TRIM da zona de Brillouin. Dessa propriedade podemos extrair a equação de vínculo

$$
x_{0} x_{1}=y_{0} y_{1}=z_{0} z_{1}=\prod_{\alpha=1}^{8} \delta\left(\Gamma_{\alpha}\right)
$$

Da equação (2.79) podemos eliminar pelo menos dois invariantes $\mathbf{Z}_{2}$ pela dependência deles por outros invariantes $\mathbf{Z}_{2}$. Assim, 

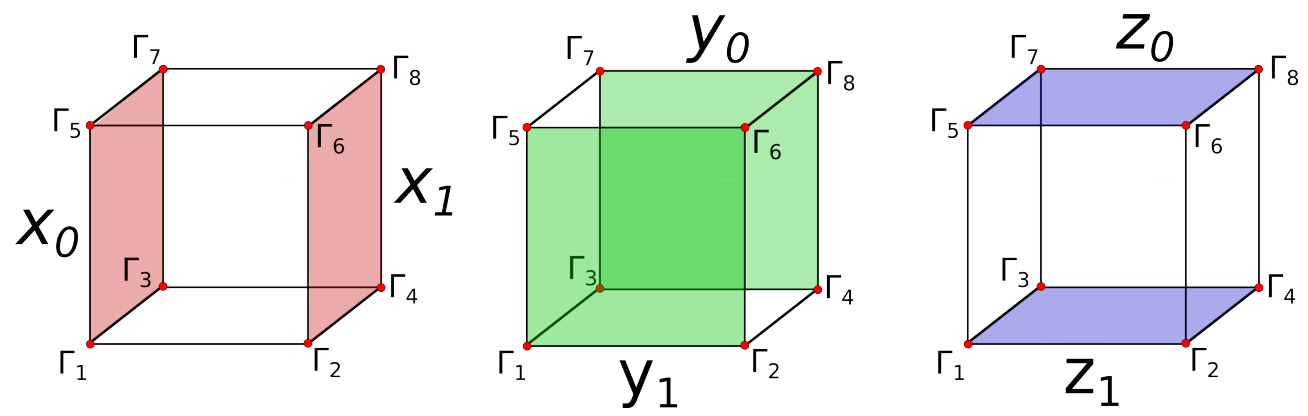

Figura 2.7: Pontos TRIM rotulados por $\Gamma_{\alpha}$, com $\alpha=1, \ldots, 8$. Planos $x_{0}, x_{1}, y_{0}, y_{1}, z_{0}$, e $z_{1}$ utilizados para calcular os invariantes $\mathbf{Z}_{2} \mathrm{em}$ materiais $3 \mathrm{D}$.

para cada material em três dimensões podemos associar o conjunto de quatro invariantes topológicos $\mathbf{Z}_{2}$ escritos como

$$
\left(\nu_{0} ; \nu_{1} \nu_{2} \nu_{3}\right)
$$

Um dos invariantes topológico é associado ao produto dos Pfaffianos em todos os TRIM, como na equação (2.79). Esse invariante topológico é utilizado para classificar os isolantes topológicos em "fracos" ou "fortes". Materiais com $\nu_{0}=1$ são chamados de isolantes topológicos fortes, enquanto que materiais com $\nu_{0}=0$ são chamados de isolantes topológicos fracos.

\subsection{Exemplos de isolantes topológicos}

Para entender as propriedades eletrônicas dos isolantes topológicos, vamos estudar alguns materiais que apresentam topologias de bandas não-triviais $(\nu \neq 0)$. Entre alguns desses materiais isolantes topológicos estão o siliceno, o germaneno, o seleneto de bismuto $\left(\mathrm{Bi}_{2} \mathrm{Se}_{3}\right)$, e o telureto de bismuto $\left(\mathrm{Bi}_{2} \mathrm{Te}_{3}\right)$. Algumas variações desses materiais serão estudados na parte de Resultados dessa tese. Para uma introdução das propriedades estruturais e eletrônicas vamos fazer primeiro uma breve descrição dos resulta- 
dos encontrados na literatura científica que precedem alguns dos nossos resultados.

\subsubsection{Siliceno}

Entre os primeiros isolantes topológicos propostos estão os baseados em grafeno [12,37]. As propriedades estruturais e eletrônicas simples dos materiais 2D serviram como base para o estudo de novas topologias de bandas não-triviais $\left(\mathrm{Z}_{2}\right)$.

Em 2010 foi descoberto o siliceno [38, 39]. Esse material apresenta uma estrutura cristalina hexagonal em duas dimensões, ilustrada na Fig. 2.8. A estrutura de bandas desse material também é mostrada na Fig. 2.8. O siliceno é um isolante topológico $2 \mathrm{D}$ com $\nu=1$ e gap de energia de aproximadamente $1,5 \mathrm{meV}$.

(a)

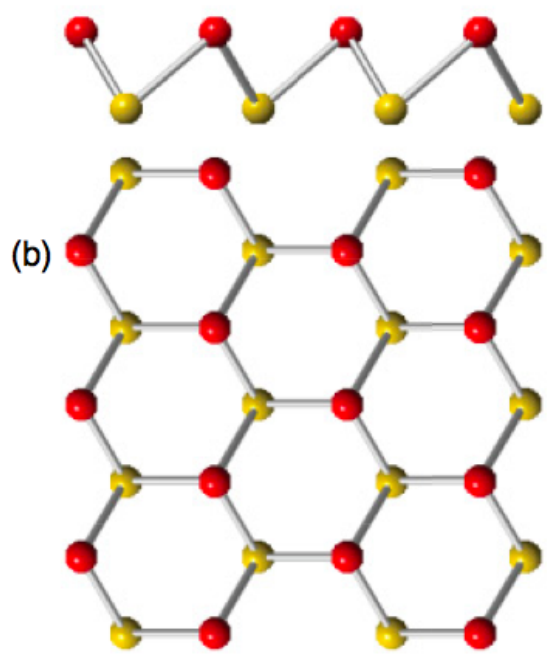

(c)
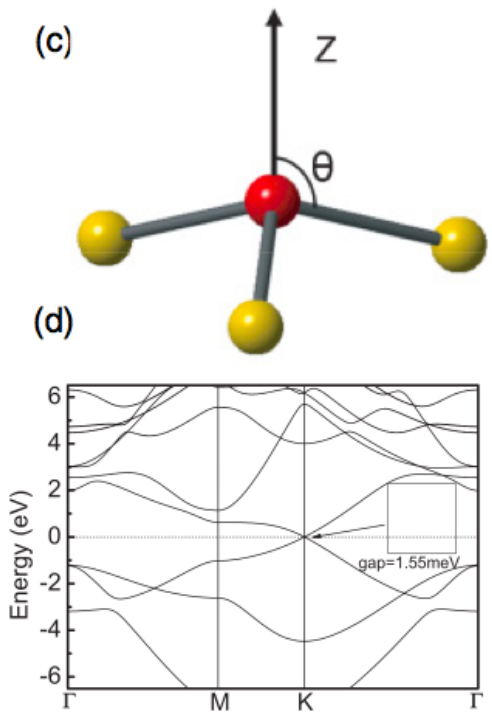

Figura 2.8: (a) Visão lateral da representação esquemática da geometria do siliceno. (b) Visão superior da represetação esquemática da geometria do siliceno. (c) Ângulos formados pela distorção da rede do siliceno. (d) Estrutura de bandas do siliceno com acoplamento spin-órbita. O material apresenta um gap de energia de $1.55 \mathrm{meV}$ no ponto $K$. Figura extraída da referência [40]. 
Outro material 2D que também é um isolante topológico é o germaneno [40]. Entretanto, as propriedades estruturais e eletrônicas desse material serão discutidas no Capítulo 5.

\subsection{2 $\mathrm{Bi}_{2} \mathbf{S e}_{3}, \mathrm{Bi}_{2} \mathbf{T e}_{3}$ e $\mathbf{S b}_{2} \mathbf{T e}_{3}$}

Entre os isolantes topológicos mais estudados estão o $\mathrm{Bi}_{2} \mathrm{Se}_{3}$, $\mathrm{Bi}_{2} \mathrm{Te}_{3}, \mathrm{e} \mathrm{Sb}_{2} \mathrm{Te}_{3}$ [18]. Esses materiais são isolantes topológicos 3D com topologia de bandas $(1 ; 000)$ [41]. As propriedades estruturais desses materiais são formadas pelo empilhamento de três camadas quíntuplas (QL, acrônimo do termo em inglês Quintuple layers) inequivalentes ligadas através da interação de van der Waals, como mostrado na Fig. 2.9.

a

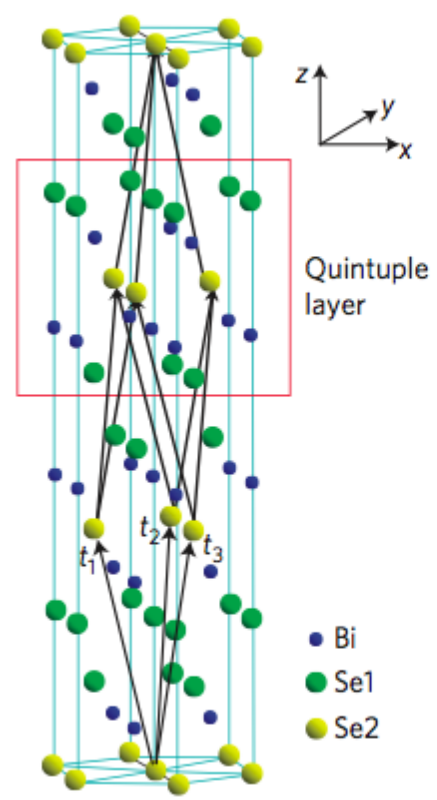

b

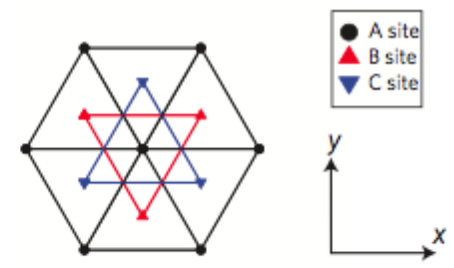

c
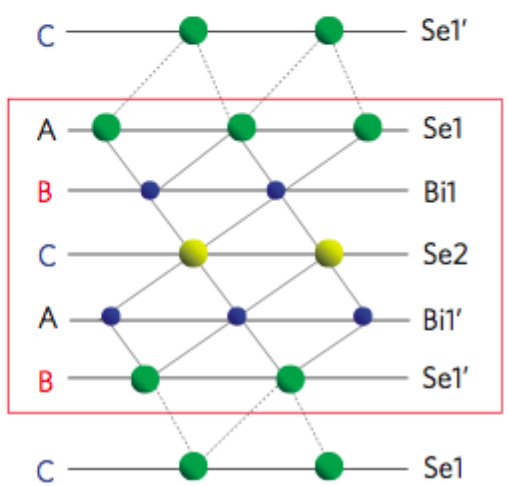

Figura 2.9: (a) Representação esquemática da geometria do $\mathrm{Bi}_{2} \mathrm{Se}_{3}$. (b) Visão superior da geometria do $\mathrm{Bi}_{2} \mathrm{Se}_{3}$. (c) Representação das camadas quíntuplas do $\mathrm{Bi}_{2} \mathrm{Se}_{3}$. Figura extraída da referência [18].

A estrutura de bandas de bulk para o $\mathrm{Bi}_{2} \mathrm{Se}_{3}$ sem acoplamento spin-órbita [ver Fig. 2.10(a)], e com acoplamento spin-órbita [ver 
Fig. 2.10(b)].
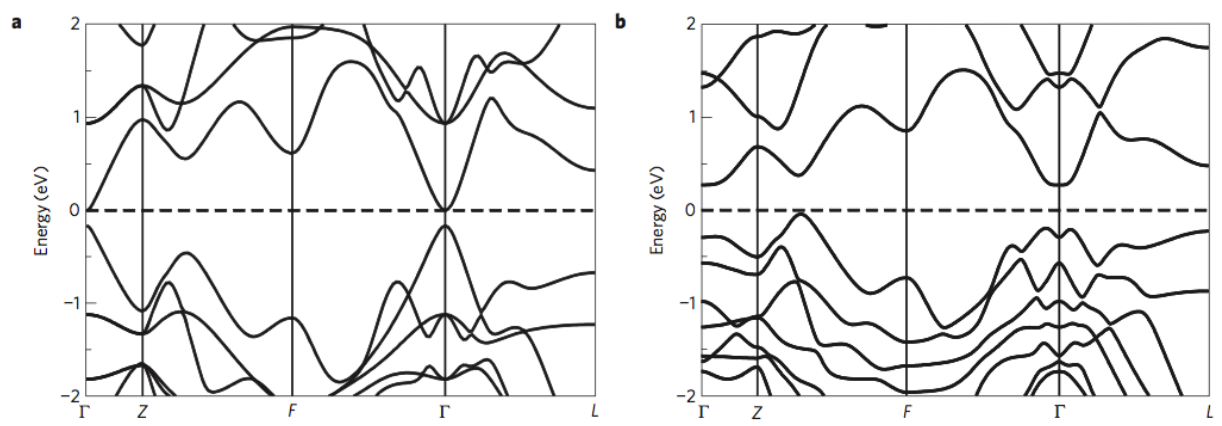

Figura 2.10: Estruturas de bandas do bulk do $\mathrm{Bi}_{2} \mathrm{Se}_{3}$ : (a) sem acoplamento spin-órbira, (b) com acoplamento spin-órbita. Figura extraída da referência [18].

As estruturas de bandas de superfíceis dos materiais $\mathrm{Sb}_{2} \mathrm{Se}_{3}$, $\mathrm{Sb}_{2} \mathrm{Te}_{3}, \mathrm{Bi}_{2} \mathrm{Se}_{3}$, e $\mathrm{Bi}_{2} \mathrm{Te}_{3}$ são mostradas na Fig. 2.11. Enquanto que o $\mathrm{Sb}_{2} \mathrm{Se}_{3}$ é um isolante trivial, os materiais $\mathrm{Bi}_{2} \mathrm{Se}_{3}, \mathrm{Bi}_{2} \mathrm{Te}_{3}$, e $\mathrm{Sb}_{2} \mathrm{Te}_{3}$ são isolantes topológicos fortes. 

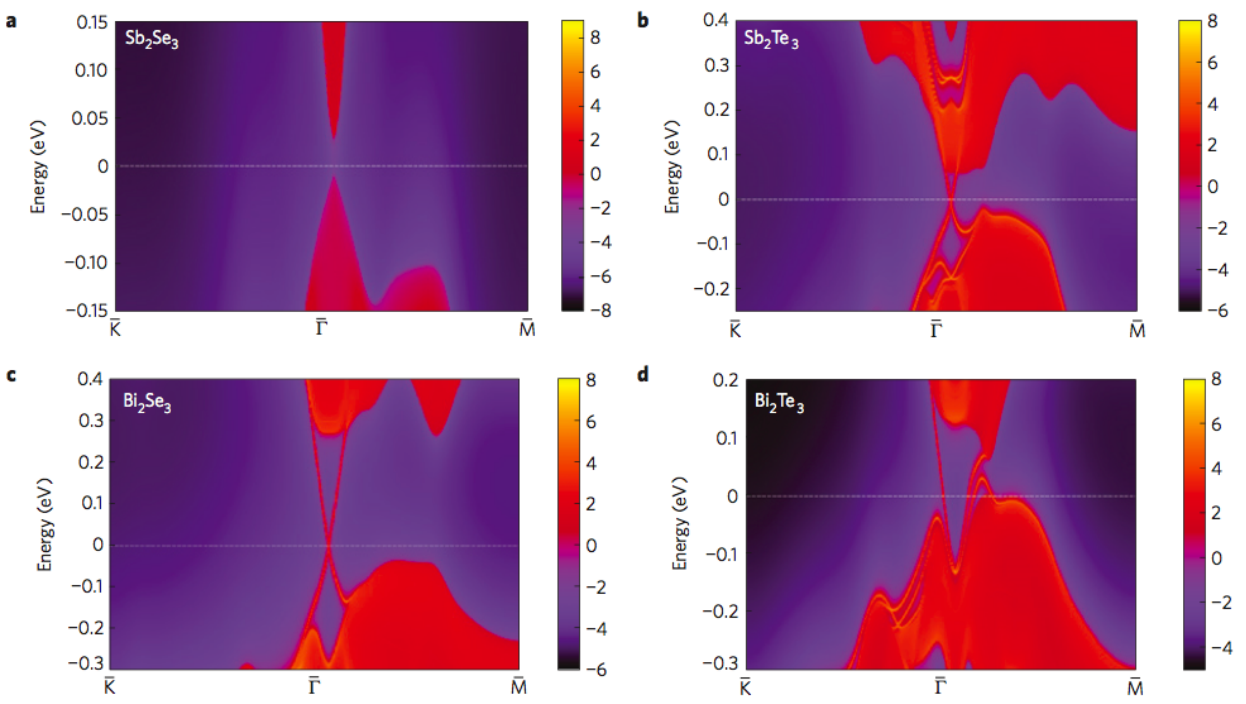

Figura 2.11: Estruturas de bandas de superfícies dos materiais: (a) $\mathrm{Sb}_{2} \mathrm{Se}_{3}$, (b) $\mathrm{Sb}_{2} \mathrm{Te}_{3}$, (c) $\mathrm{Bi}_{2} \mathrm{Se}_{3}$, e (d) $\mathrm{Bi}_{2} \mathrm{Te}_{3}$. Figura extraída da referência [18]. 


\section{Parte II}

\section{Resultados}





\section{Falhas de empilhamentos em $\mathrm{Bi}_{2} \mathrm{Se}_{3}$ e $\mathrm{Bi}_{2} \mathrm{Te}_{3}$}

The greatest of faults, I

should say, is to be conscious

of none.

Thomas Carlyle

\subsection{Introdução}

Devido ao grande interesse nas propriedades estruturais e eletrônicas dos isolantes topológicos, vamos investigar neste Capítulo os isolantes topológicos $\mathrm{Bi}_{2} \mathrm{Se}_{3}$ e $\mathrm{Bi}_{2} \mathrm{Te}_{3}$ com defeitos do tipo falhas de empilhamentos. Esse tipo de defeito está presente em vários materiais e podem modificar localmente as propriedades estruturais de forma que crie uma pequena região de "vácuo" na falha. Dependendo do tamanho dessa região de "vácuo" e das propriedades eletrônicas dos bulks dos isolantes topológicos, é possivel surgir estados metálicos nas proximidades do defeito. 


\subsection{Propriedades estruturais}

De início, é importante recordar que os bulks de $\mathrm{Bi}_{2} \mathrm{Se}_{3}$ e $\mathrm{Bi}_{2} \mathrm{Te}_{3}$ são formados por empilhamentos de três tipos de camadas quíntuplas inequivalentes, como mostrado na Fig. 3.1. Essas camadas quíntuplas foram denotadas por $A, B$ e $C$, e devem ser empilhadas em sequências periódicas ...ABCABC ... para formar bulks pristinos, ou seja, sem defeitos. As regiões dos cristais de $\mathrm{Bi}_{2} \mathrm{Se}_{3}$ e $\mathrm{Bi}_{2} \mathrm{Te}_{3}$ que não seguem esse padrão de empilhamento são caracterizadas como falhas de empilhamentos.

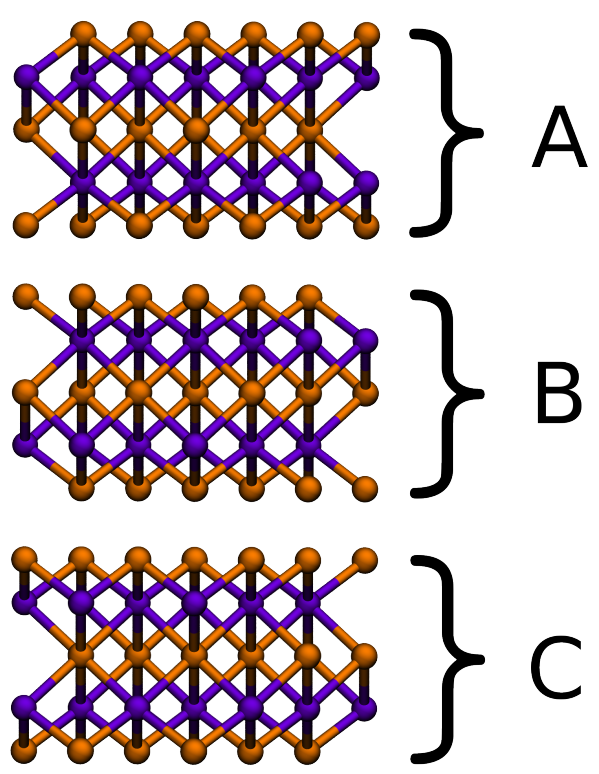

(a)
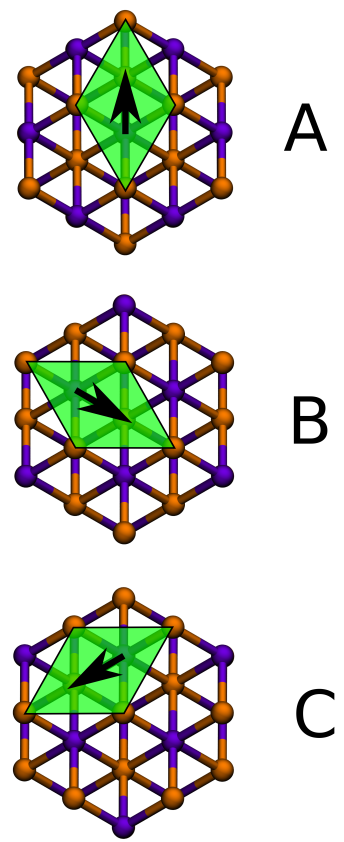

(b)

Figura 3.1: (a) Represetação esquemática da geometria do $\mathrm{Bi}_{2} \mathrm{Se}_{3}$ com as três $\mathrm{QL}$ inequivalentes. Essas três $\mathrm{QL}$ inequivalentes são rotuladas de A, B e C. (b) Visões superiores das orientações das QLS inequivalentes como rotações de $120^{\circ}$ em torno do eixo perpendicular ao plano de empilhamento. Uma célula unitária é mostrada hachurada em verde, com as setas pretas para indicar as rotações de $120^{\circ}$ em torno do eixo $z$.

Devido à natureza mais fraca das ligações de van der Waals 
quando comparadas as ligações covalentes, é esperado que no processo de crescimento dos cristais de $\mathrm{Bi}_{2} \mathrm{Se}_{3}$ e $\mathrm{Bi}_{2} \mathrm{Te}_{3}$ haja uma grande probabilidade de formação de falhas de empilhamentos entre as camadas quíntuplas.

Para o estudo das formações de falhas de empilhamentos em cristais de $\mathrm{Bi}_{2} \mathrm{Se}_{3}$ e $\mathrm{Bi}_{2} \mathrm{Te}_{3}$ foram utilizados cálculos de primeiros princípios baseados na Teoria do Funcional da Densidade [42, 43] conforme implementado no código computacional VASP [44, 45]. Devido a interação entre camadas quíntuplas, foram utilizados funcionais de troca-correlação LDA [46,47] para o cálculo das propriedades estruturais e energéticas. Foram utilizadas energias de cortes de $212 \mathrm{eV}$ para o $\mathrm{Bi}_{2} \mathrm{Se}_{3}$ e $175 \mathrm{eV}$ para $\mathrm{Bi}_{2} \mathrm{Te}_{3}$, amostragens de pontos- $k$, na zona de Brillouin, seguindo o esquema proposto por Monkhorst-Pack [48] com matrizes diagonais $5 \times 5 \times 1$. Também foram utilizados potenciais PAW, conforme descrito em [49, 50].

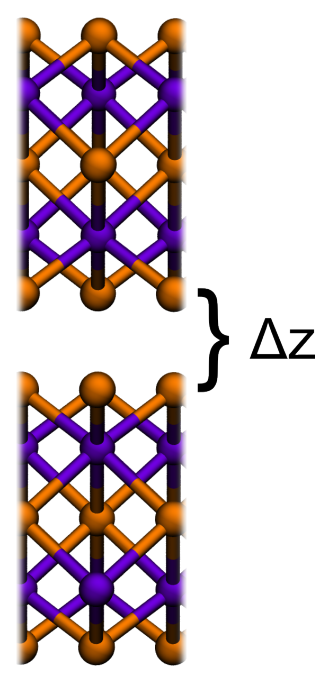

(a)
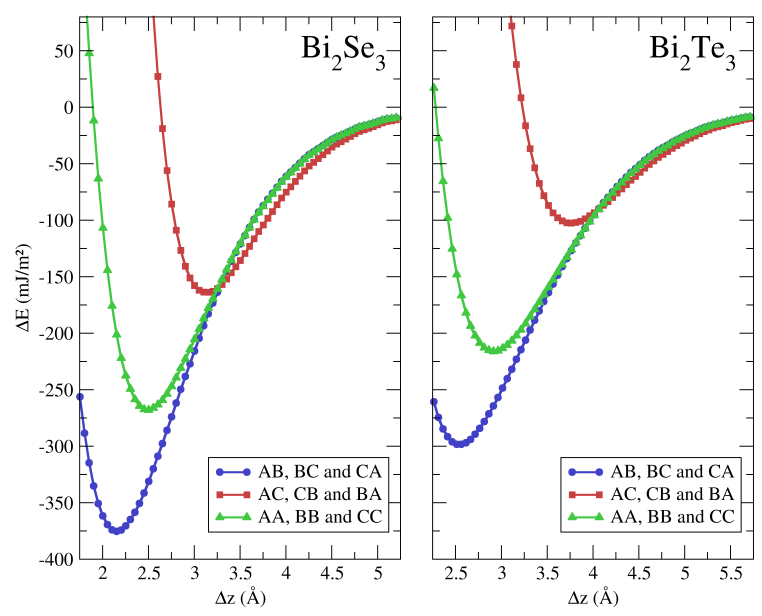

(b)

Figura 3.2: (a) Representação esquemática da separação $\Delta z$ entre duas QL. (b) Curvas de energias de ligações em funções das distâncias entre $\mathrm{QL}$ para $\mathrm{Bi}_{2} \mathrm{Se}_{3} \mathrm{e} \mathrm{Bi}_{2} \mathrm{Te}_{3}$.

A interação entre QL foi estudada primeiramente através de um 
modelo composto por duas QL. A separação em $z, \Delta z$, entre as QL foi variada em torno do valor experimental do sistema pristino, $\Delta z_{\text {exp }}=2.25 \AA$ para o $\mathrm{Bi}_{2} \mathrm{Se}_{3}$ e $\Delta z_{\text {exp }}=2.76 \AA$ para o $\mathrm{Bi}_{2} \mathrm{Te}_{3}$. As curvas obtidas por essas separações são mostradas na Fig. 3.2. Foi observado que as separações em $z$ para empilhamentos do tipo ( $B A, A C, C B$ ) foram de $\Delta z_{\text {min }}=3.25 \AA$ para o $\mathrm{Bi}_{2} \mathrm{Se}_{3}$ e $\Delta z_{\text {min }}=3.96$ $\AA$ para o $\mathrm{Bi}_{2} \mathrm{Te}_{3}$; e para empilhamentos do tipo $(A A, B B, C C)$ as separações em $z$ encontradas foram de $\Delta z_{\min }=2.50 \AA$ para o $\mathrm{Bi}_{2} \mathrm{Se}_{3}$ e $\Delta z_{\min }=3.01 \AA$ para $\mathrm{Bi}_{2} \mathrm{Te}_{3}$.

A partir das separações em $z$ encontradas foram construídas as geometrias dos cristais de $\mathrm{Bi}_{2} \mathrm{Se}_{3}$ e $\mathrm{Bi}_{2} \mathrm{Te}_{3}$ com falhas de empilhamentos. A exclusão de uma QL no bulk pristino faz com que tenhamos uma falha de empilhamento intrínseca (ISF, acrônimo do termo em inglês Intrinsec Stacking Fault), que é reminiscente dos empilhamentos entre QL do tipo $B A, A C$ e $C B$. Além da exclusão de QL, podemos criar falhas de empilhamentos a partir da inserção de uma QL do sistema pristino. A inserção de uma QL do bulk pristino dá origem à uma falha de empilhamento extrínseca (ESF, acrônimo do termo em inglês Extrinsec Stacking Fault), que é reminiscente dos empilhamentos entre QL do tipo $A A, B B$ e $C C$. Representações esquemáticas das falhas de empilhamentos intrínsecas e extrínsecas no $\mathrm{Bi}_{2} \mathrm{Se}_{3}$ e $\mathrm{Bi}_{2} \mathrm{Te}_{3}$ são mostradas na Fig. 3.3.

A partir das curvas de interações entre QL também podemos obter as energias das falhas de empilhamentos, que são as energias necessárias para a formação desse tipo de defeito nos cristais pristinos. Para o ISF e ESF do $\mathrm{Bi}_{2} \mathrm{Se}_{3}$ foram obtidas as energias de falhas de empilhamentos $\gamma_{I S F}=212 \mathrm{~mJ} / \mathrm{m}^{2}$ e $\gamma_{E S F}=107 \mathrm{~mJ} / \mathrm{m}^{2}$, enquanto que para os ISF e ESF do $\mathrm{Bi}_{2} \mathrm{Te}_{3}$ foram obtidas $\gamma_{I S F}=$ $196 \mathrm{~mJ} / \mathrm{m}^{2}$ e $\gamma_{E S F}=82 \mathrm{~mJ} / \mathrm{m}^{2}$. Essas energias de falhas de empilhamentos são compatíveis com de outras estruturas, como: $\gamma_{I S F}^{N i}=$ $187 \mathrm{~mJ} / \mathrm{m}^{2}, \gamma_{E S F}^{N i}=149 \mathrm{~mJ} / \mathrm{m}^{2}$ [51, 52]; $\gamma_{I S F}^{S i}=33 \mathrm{~mJ} / \mathrm{m}^{2}, \gamma_{E S F}^{S i}=$ $26 \mathrm{~mJ} / \mathrm{m}^{2}$ [51]; e $\gamma_{I S F}^{R h}=750 \mathrm{~mJ} / \mathrm{m}^{2}$ e $\gamma_{E S F}^{R h}=291 \mathrm{~mJ} / \mathrm{m}^{2}$ [51,52]. 


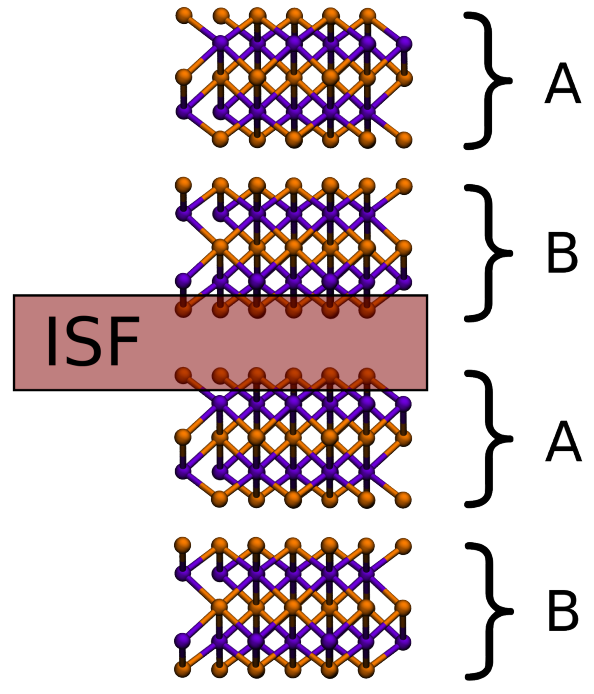

(a)

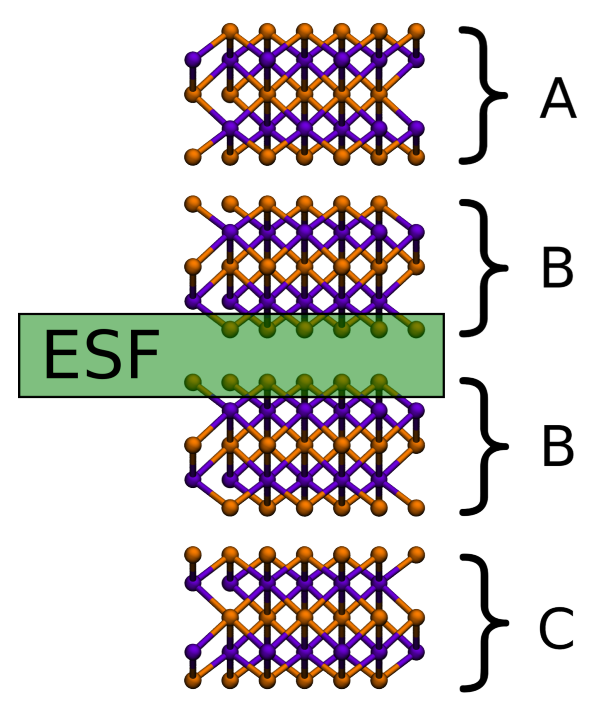

(b)

Figura 3.3: Representações esquemáticas das falhas de empilhamentos em $\mathrm{Bi}_{2} \mathrm{Se}_{3}$ e $\mathrm{Bi}_{2} \mathrm{Te}_{3}$ : (a) Falha de empilhamento intrínseca (ISF) com a região de ligações mais fracas entre QL hachurada em vermelho, (b) Falha de empilhamento extrínseca (ESF) com a região de ligações mais fracas entre QL hachurada em verde. 


\subsection{Propriedades eletrônicas}

As propriedades eletrônicas dos bulk de $\mathrm{Bi}_{2} \mathrm{Se}_{3}$ e $\mathrm{Bi}_{2} \mathrm{Te}_{3}$ na presença de falhas de empilhamentos foram estudadas a partir da estrutura de bandas em zonas de Brillouin $2 D$, devido à característica $2 D$ das falhas de empilhamentos. As estruturas de bandas encontradas para o ISF e ESF em $\mathrm{Bi}_{2} \mathrm{Se}_{3}$ são mostradas na Fig. 3.4, e para o ISF e ESF em $\mathrm{Bi}_{2} \mathrm{Te}_{3}$ são mostradas na Fig. 3.5.
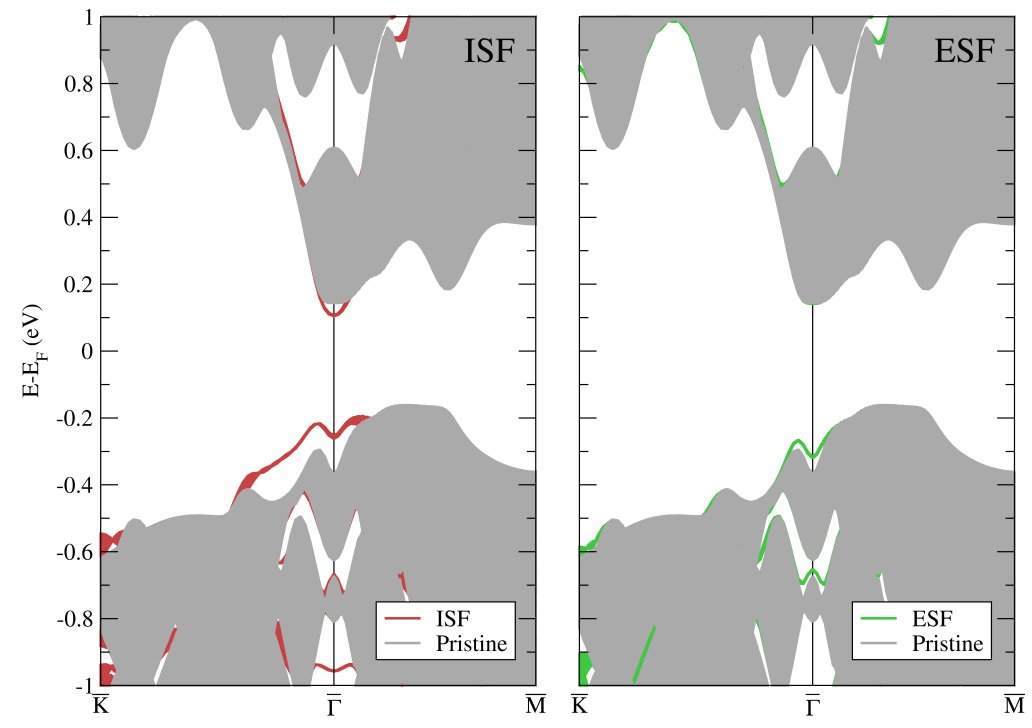

Figura 3.4: Estrutura de bandas das falhas de empilhamentos em $\mathrm{Bi}_{2} \mathrm{Se}_{3}$ no bulk, ISF (painel à esquerda) e ESF (painel à direita). Os estados das falhas de empilhamentos intrínsecas são representados em vermelho no painel à esquerda, enquanto que os estados das falhas de empilhamentos extrínsecas são representados em verde no painel à direita. As bandas de bulk são projetadas em cinza.

As falhas de empilhamentos no $\mathrm{Bi}_{2} \mathrm{Se}_{3}$ criam estados acima das bandas de valência, e abaixo da banda de condução. A redução no gap de energia no ponto $\Gamma$ do bulk é de $\Delta^{\Gamma}=0.51 \mathrm{eV}$, para $\Delta_{\Gamma}^{I S F}=0.36 \mathrm{eV}$ no caso do ISF e $\Delta_{\Gamma}^{E S F}=0.46 \mathrm{eV}$ no caso do ESF. 

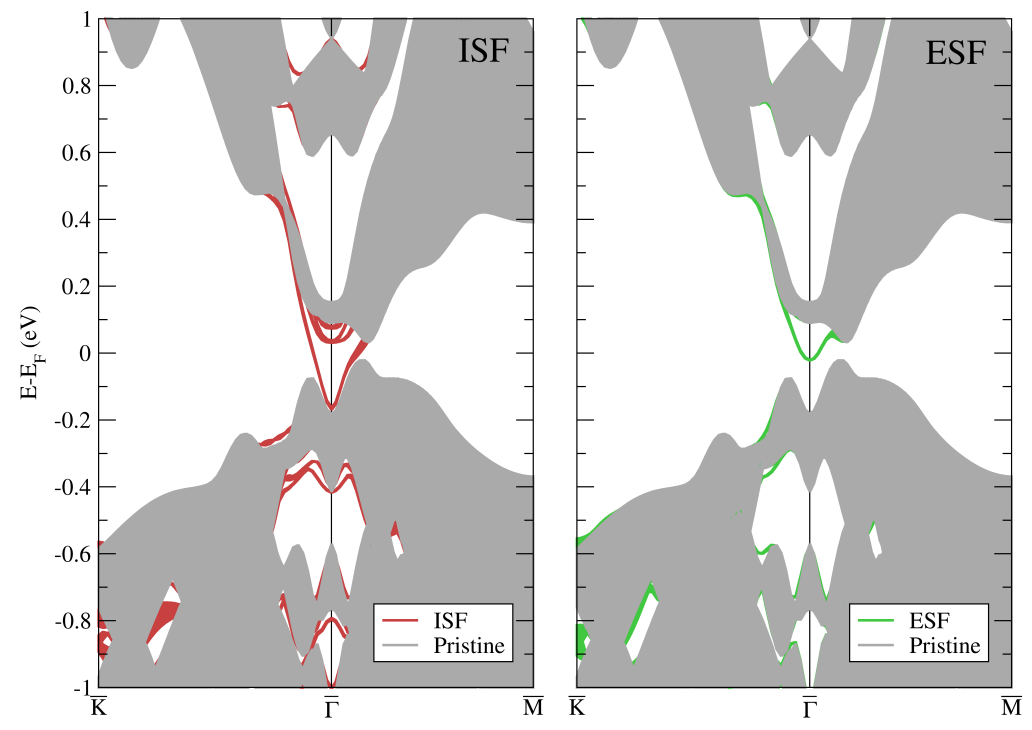

Figura 3.5: Estrutura de bandas das falhas de empilhamentos em $\mathrm{Bi}_{2} \mathrm{Te}_{3}$ no bulk, ISF (painel à esquerda) e ESF (painel à direita). Os estados das falhas de empilhamentos intrínsecas são representados em vermelho no painel à esquerda, enquanto que os estados das falhas de empilhamentos extrínsecas são representados em verde no painel à direita. As bandas de bulk são projetadas em cinza. 
Para as falhas de empilhamentos nos bulk de $\mathrm{Bi}_{2} \mathrm{Te}_{3}$, foram observados que os estados provenientes das falhas de empilhamentos tornam o material metálico. Essa transição para estados metálicos surge no $\mathrm{Bi}_{2} \mathrm{Te}_{3}$ devido ao grande espaçamento entre $\mathrm{QL}$ causado pela falha de empilhamento e ao pequeno gap do bulk pristino de $\mathrm{Bi}_{2} \mathrm{Te}_{3}$.

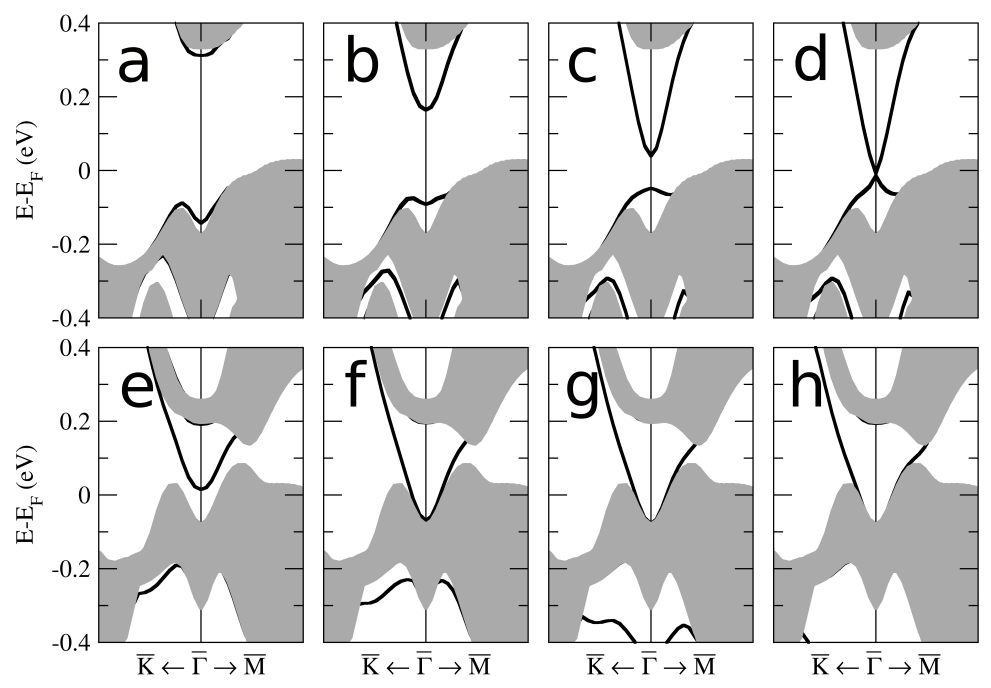

Figura 3.6: Estrutura de bandas do $\mathrm{Bi}_{2} \mathrm{Se}_{3}$ com espaçamentos adicionais: (a) $0.35 \AA$, (b) $1.00 \AA$, (c) $2.00 \AA \AA$, (d) $5.00 \AA$; e para o $\mathrm{Bi}_{2} \mathrm{Te}_{3}$ com espaçamentos adicionais: (e) $0.35 \AA$, (f) $0.70 \AA \AA$, (g) $1.20 \AA$, (h) 5.00 $\AA$.

Para entender o processo de formação dos estados das falhas de empilhamentos, foram calculadas as estruturas de bandas do bulk pristino de $\mathrm{Bi}_{2} \mathrm{Se}_{3}$ e $\mathrm{Bi}_{2} \mathrm{Te}_{3}$ com um espaçamento adicionais entre duas QL no bulk. Na Fig. 3.6, temos as bandas de bulk com um dos espaçamentos adicionais entre QL de 0, $0.35 \AA$ A, $1.00 \AA$, 2.00 $\AA$ e $5.00 \AA$ em relação à separação experimental para o $\mathrm{Bi}_{2} \mathrm{Se}_{3}$, e espaçamentos de $0,0.35 \AA, 0.70 \AA, 1.20 \AA$ e $5.00 \AA$ em relação à separação experimental para o $\mathrm{Bi}_{2} \mathrm{Te}_{3}$. Os espaçamentos adicionais são realizados nas geometrias do $\mathrm{Bi}_{2} \mathrm{Se}_{3} \mathrm{e} \mathrm{Bi}_{2} \mathrm{Te}_{3}$ sem defeitos do 
tipo falhas de empilhamentos.

\subsection{Falhas de empilhamentos próximas às superficies}

As falhas de empilhamentos no $\mathrm{Bi}_{2} \mathrm{Se}_{3}$ e $\mathrm{Bi}_{2} \mathrm{Te}_{3}$ podem aparecer tanto no bulk como nas proximidades das superfícies dos materiais. Além das propriedades eletrônicas das falhas de empilhamentos nos bulks, foram calculadas também as propriedades eletrônicas dos estados das falhas de empilhamentos e estados de superficies quando as falhas de empilhamentos estão próximas às superfícies. Os efeitos de proximidades ocorrem entre a primeira e segunda QL do slab que é usado para modelar as superfícies de $\mathrm{Bi}_{2} \mathrm{Se}_{3}$ e $\mathrm{Bi}_{2} \mathrm{Te}_{3}$. As estruturas de bandas encontradas são mostradas na Fig. 3.7.

Como podemos ver na estrutura de bandas do $\mathrm{Bi}_{2} \mathrm{Se}_{3}$, as falhas de empilhamentos levam a um deslocamento do cone de Dirac em relação ao cone de Dirac do sistema pristino. Dessa forma, podemos ver que a presença das falhas de empilhamentos podem resolver ou amenizar o problema da presença de estados de bulk que podem causar espalhamentos reversos, e assim, facilitar a localização dos estados eletrônicos por desordens.

\subsection{Conclusão}

No estudo das falhas de empilhamentos em isolantes topológicos do tipo $\mathrm{Bi}_{2} \mathrm{Se}_{3}$ e $\mathrm{Bi}_{2} \mathrm{Te}_{3}$ através de cálculos de primeiros princípios, foram observadas que as energias de formações são da mesma ordem de grandeza das encontradas em outros materiais como Si, Ni e Rh [51,52].

As falhas de empilhamentos causam espaçamentos entre QL que podem imitar a formação de superficies e criarem estados me- 

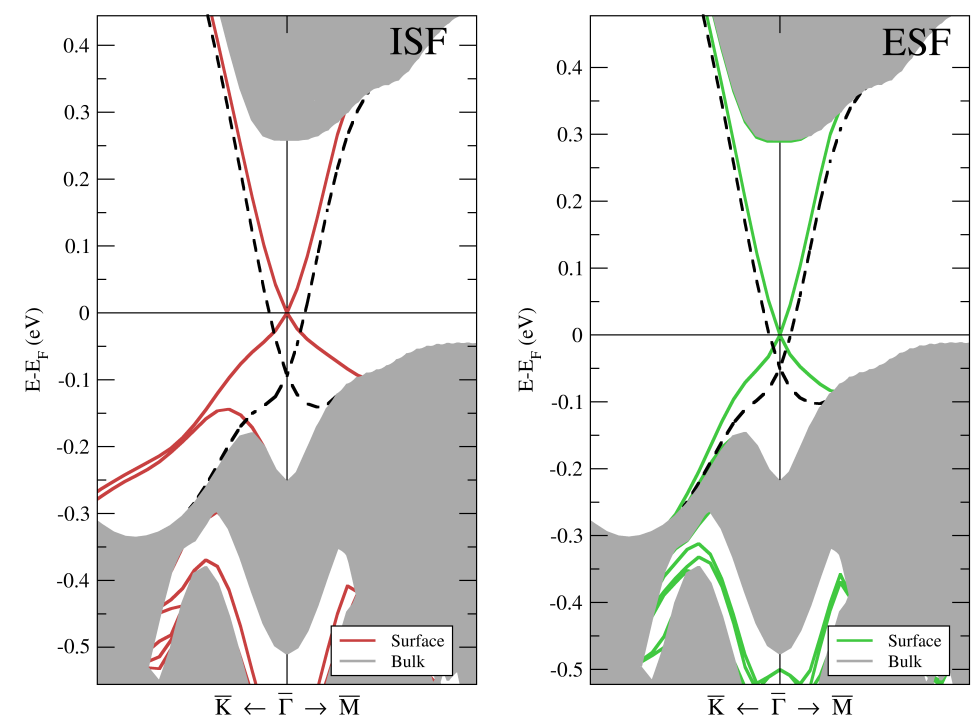

Figura 3.7: Estrutura de bandas do $\mathrm{Bi}_{2} \mathrm{Se}_{3}$ com falhas de empilhamentos próximos às superfícies. Os bandas em vermelho (painel à esquerda) estão associadas aos estados de superfície do isolante topológico na proximidade de uma falha de empilhamento intrínseca. O ponto de Dirac é deslocado em torno de $0.1 \mathrm{eV}$ em relação ao cone de Dirac do sistema pristino (tracejado em preto). As bandas em verde (no painel à direita) estão associadas aos estados de superfícies do isolante topológico na proximidade de uma falha de empilhamento extrínseca. O ponto de Dirac é deslocado em torno de 0.05 eV em relação ao cone de Dirac do sistema pristino. 
tálicos no bulk. Esses estados metálicos não alteram as propriedades topológicas dos cones de Dirac da superfície. Entretanto, podem alterar as propriedades de transporte e as propriedades mecânicas.

Nas proximidades das superfícies dos isolantes topológicos, a presença das falhas de empilhamentos pode alterar a localização dos cones de Dirac, que também devem contribuir nas modificações das propriedades de transporte eletrônico. 


\title{
Interfaces entre isolantes topológicos e semicondutores
}

\author{
A model is a lie that helps \\ you see the truth.
}

Howard Skipper

\subsection{Introdução}

Uma das principais características dos isolantes topológicos é a presença de estados de superfícies topologicamente protegidos contra perturbações que respeitem a simetria de reversão temporal (TRS, acrônimo do termo em inglês Time Reversal Symmetry) [21,53]. Além de estados de superfície, os isolantes topológicos podem apresentar estados de interface entre TI e semicondutores ou isolantes. Embora os estados de superfícies sejam bem caracterizados por técnicas experimentais como ARPES [20, 54], STM [55] e NMR [56], os estados de interface ainda foram poucos estudados. As interações entre os estados topológicos dos isolantes topológicos e os estados topologicamente triviais dos semicondutores (banda de valência ou banda de condução) podem levar à desdobramentos do cone de Dirac e mudanças nas texturas de spin mesmo na ausên- 
cia de acoplamentos que quebrem a simetria de reversão temporal. Esse tipo de desdobramento deve conter degenerescências de Kramers nos momentos invariantes por reversão temporal (TRIM, acrônimo do termo em inglês Time-Reversal Invariant Momenta). Ao contrário de dopagens magnéticas [57-59] ou formações de ligas entre isolantes topológicos e isolantes triviais [60], esse fenômeno pode ocorrer sem transições topológicas das bandas de bulk. A estrutura eletrônica e texturas de spin dos estados de interfaces serão estudados a partir de um modelo Hamiltoniano efetivo e a partir de cálculos de primeiros princípios baseados na Teoria do Funcional da Densidade (ver Apêndice A).

Os fênomenos eletrônicos que ocorrem na interface entre os isolantes topológicos e os semicondutores (TI/S) podem ser fundamentalmente importantes para o desenvolvimento de dispositivos spintrônicos. Em especial, para os transistores spintrônicos, o longo comprimento de coerência de spin do GaAs e a geração de correntes de spin dos isolantes topológicos [22] podem agir em aplicações lógicas dos transistores. O entendimento mais profundo dos estados topológicos de interfaces podem guiar o desenvolvimento de novos dispositivos e novas aplicações.

Entre os isolantes topológicos mais conhecidos estão os calcogenetos de pnictogênios, tais como $\mathrm{Bi}_{2} \mathrm{Se}_{3}, \mathrm{Bi}_{2} \mathrm{Te}_{3}, \mathrm{Sb}_{2} \mathrm{Te}_{3}$ [18]. Os acoplamentos spin-órbita relativamente grandes e gaps de energia da ordem de $0,2-0,3 \mathrm{eV}$ fazem desses materiais uma plataforma interessante para estudar novas topologias de bandas não-triviais. A estrutura atômica desses materiais são formadas por camadas quíntuplas (QL, acrônimo do termo em inglês Quintuple Layers) fracamente ligadas através de interações de van der Waals. Esse tipo de estrutura favorece a formação de superfícies (0001) sem ligações pendentes. Essa superfície é a de energia de superfície mais baixa. Esses isolantes topológicos podem ser crescidos em substratos semicondutores como GaAs [61,62], Si [63] ou InP [64]. Para alguns substratos, podemos usar o método de crescimento epita- 
xial metalorgânico de van der Waals. Nesse método, moléculas são depositadas quimicamente em um substrato de forma que o cristal de isolante topológico cresça camada por camada. A denominação de van der Waals desse tipo de crescimento vem do surgimento de ligações de van der Waals entre o cristal em crescimento e o substrato. Esse tipo de crescimento também pode alterar as últimas camadas atômicas do substrato semicondutor. Em especial para o GaAs, substituições de calcogênios (Se ou Te) nos sítios de As podem passivar (ou tratar) a superfície terminada em As do GaAs,

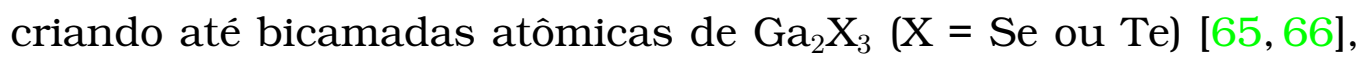
como mostrado na Fig. 4.1. Nós podemos esperar que diferentes passivações de Se do GaAs podem gerar diferentes propriedades eletrônicas para as interfaces de $\mathrm{Bi}_{2} \mathrm{Se}_{3} / \mathrm{GaAs}$.

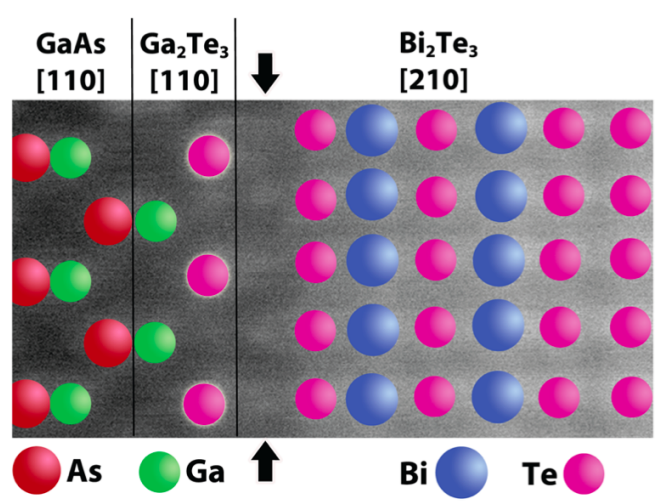

Figura 4.1: Imagem de STM da interface entre os materiais GaAs e $\mathrm{Bi}_{2} \mathrm{Te}_{3}$, formando uma bicamada atômica de $\mathrm{Ga}_{2} \mathrm{Te}_{3}$. Figura extraída da referência [61].

\subsection{Modelo Hamiltoniano efetivo}

Nosso desenvolvimento em direção ao entendimento das propriedades eletrônicas de interfaces isolantes topológicos e semicondutores podem ser iniciados a partir de um modelo Hamiltoniano efetivo onde os estados topológicos do isolante topológico interagem 
com as bandas parabólicas topologicamente triviais do semicondutor. Nesse sentido, o nosso modelo será conduzido a entender a interação eletrônica entre esses dois materiais. As propriedades eletrônicas dos isolantes topológicos e semicondutores livres serão tomados como ponto de partida para o modelo. Note que esse modelo não é um modelo tight-binding que parte dos orbitais atômicos e integrais de hoppings para descrever as propriedades eletrônicas de um material (livre).

Os estados topológicos do isolante topológico pristino são dados por um cone de Dirac com relação de dispersão linear e texturas de spin helicoidal (relação fixa entre spin e momento) de forma que na base $|\psi\rangle=\left(\left|\psi_{\text {TI }}^{\uparrow}\right\rangle\left|\psi_{\text {TI }}^{\downarrow}\right\rangle\right)^{T}$ seja escrito por $v_{F} \hbar(\mathbf{k} \times \boldsymbol{\sigma}) \cdot \hat{\mathbf{z}}$. A velocidade de Fermi $v_{F}=5 \cdot 10^{5} \mathrm{~m} / \mathrm{s}$ descreve a inclinação do cone de Dirac para o $\mathrm{Bi}_{2} \mathrm{Se}_{3}$. Esse tipo de Hamiltoniano é semelhante ao Hamiltoniano $v_{F} \hbar \mathbf{k} \cdot \boldsymbol{\sigma}$ das referências [12], mas com a textura de spin helicoidal.

Os estados dos semicondutores (banda de valência ou banda de condução) são degenerados em spin e descritos por parábolas onde a massa efetiva é definida pela concavidade da banda $\left(\frac{1}{m_{*}}=\frac{1}{\hbar^{2}} \frac{\partial^{2} E}{\partial k^{2}}\right.$. $)$ para buracos/elétrons. Para o GaAs, a massa efetiva de buracos usada é de $m_{*}=-0.456 m_{0}$. Essa massa efetiva equivale a massa efetiva de buracos pesados encontrados experimentalmente, $m_{h h}=-0.45 m_{0}$. Se a interação do isolante topológico fosse com os buracos leves do GaAs, a massa efetiva seria de aproximadamente $m_{l h}=-0.082 m_{0}$. O valor utilizado no modelo $\left(m_{*}=-0.456 m_{0}\right)$ foi calculado através de métodos de primeiros princípios de uma superfície de GaAs livre (na ausência de interações com o isolante topológico). Além da massa efetiva, outro parâmetro utilizado é o valor do VBM (CBM) da banda de valência (condução) em relação ao ponto de Dirac do TI, $\Delta$. Em nosso cálculos, estimamos que $\Delta=0.05 \mathrm{eV}$. Usando a base $\left|\psi_{S}\right\rangle=\left(\left|\psi_{\mathrm{S}}^{\uparrow}\right\rangle\left|\psi_{\mathrm{S}}^{\downarrow}\right\rangle\right)^{T}$, o Hamiltoniano efetivo para o semicondutor é $\left(\frac{\hbar^{2}}{m_{*}}|\mathbf{k}|^{2}+\Delta\right) 1_{2 \times 2}$, onde $1_{2 \times 2}$ representa a degenerescência de spin do semicondutor. Dado que em geral 
os isolantes topológicos 3D possuem gaps de energias em torno de $0,2-0,3 \mathrm{eV}$, a janela de energia em que os cones de Dirac interagem com o semicondutor geralmente se restringe a uma única banda desse material (a banda de valência ou a banda de condução). Esse tipo de interação pode ser encontrada em interfaces tipo-II ou tipo-III, como mostrado na Fig. 4.2.

a

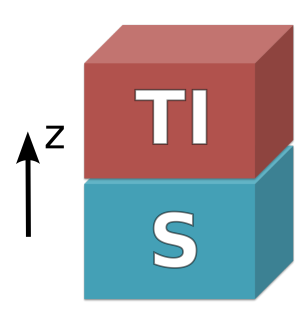

b

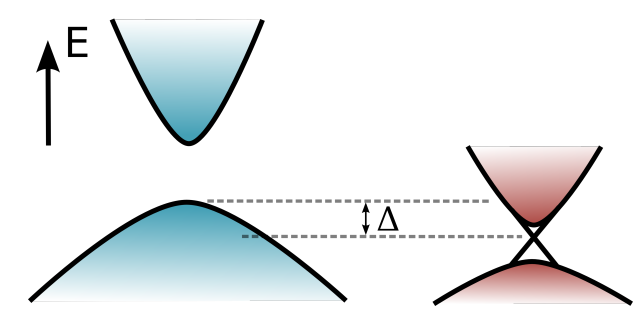

Figura 4.2: (a) Representação esquemática de uma interface tipo-III entre um isolante topológico (TI) e um semicondutor (S). (b) Representações esquemáticas das estrutura de bandas de um semicondutor (em azul) e um isolante topológico (em vermelho). O parâmetro $\Delta$ no modelo Hamiltoniano é o valor relativo do VBM em relação ao ponto de Dirac.

Para modelar a interface utilizamos a base

$$
|\psi\rangle=\left(\begin{array}{c}
\left|\psi_{\mathrm{TI}}^{\uparrow}\right\rangle \\
\left|\psi_{\mathrm{TI}}^{\downarrow}\right\rangle \\
\left|\psi_{\mathrm{S}}^{\uparrow}\right\rangle \\
\left|\psi_{\mathrm{S}}^{\downarrow}\right\rangle
\end{array}\right),
$$

fornecendo o Hamiltoniano

$$
H(\mathbf{k})=\left(\begin{array}{cc}
v_{F} \hbar(\mathbf{k} \times \boldsymbol{\sigma}) \cdot \hat{\mathbf{z}} & V_{\text {int }} \\
V_{\text {int }}^{\dagger} & \left(\frac{\hbar^{2}}{2 m_{*}}|\mathbf{k}|^{2}+\Delta\right) \mathbb{1}_{2 \times 2}
\end{array}\right) .
$$

Sem perdas de generalidade, podemos escolher o ponto de Dirac do isolante topológico como zero.

A interação entre o isolante topológico e o semicondutor é parametrizada por quatro parâmetros complexos: $\alpha_{0}, \alpha_{1}, \alpha_{2}$, e $\alpha_{3}$, na 
forma

$$
V_{\text {int }}=\alpha_{0} \mathbb{1}_{2 \times 2}+\sum_{i=1}^{3} \alpha_{i} \sigma_{i},
$$

onde $\sigma_{1}, \sigma_{2}$ e $\sigma_{3}$ são as matrizes de Pauli.

Os caráteres topológicos dos estados de Bloch são diretamente manifestados como uma textura de spin calculada pelo valor esperado das matrizes $4 \times 4$ :

$$
\Sigma_{i}=\frac{\hbar}{2} 1_{2 \times 2} \otimes \sigma_{i}, \quad i=1,2,3 .
$$

Já que o sistema não possui momentos magnéticos locais ou ordens magnéticas, a simetria de reversão temporal é obedecida. No nosso modelo, essa simetria pode ser entendida a partir da atuação do operador de reversão temporal escrito como

$$
\Theta=i \hat{K} 1_{2 \times 2} \otimes \sigma_{2}
$$

onde $\hat{K}$ é o operador de conjugação complexa. $\Theta$ é um operador anti-unitário, que obedece a equação

$$
\Theta^{2}=-1
$$

Considerando a atuação desse operador no Hamiltoniano de interação

$$
H_{\text {int }}=\left(\begin{array}{cc}
0 & V_{\text {int }} \\
V_{\text {int }}^{\dagger} & 0
\end{array}\right),
$$

vamos obter que

$$
\left[\Theta, H_{\mathrm{int}}\right]=0 \Rightarrow \sigma_{2} V_{\mathrm{int}}^{*}=V_{\mathrm{int}} \sigma_{2}
$$


Substituindo a equação (4.3) na equação (4.8), obtemos

$$
\begin{gathered}
\alpha_{0}=\alpha_{0}^{*}, \\
\alpha_{1}=-\alpha_{1}^{*}, \\
\alpha_{2}=-\alpha_{2}^{*}, \\
\alpha_{3}=-\alpha_{3}^{*} .
\end{gathered}
$$

Ou seja, o parâmetro $\alpha_{0}$ é real e os parâmetros $\alpha_{1}, \alpha_{2}$ e $\alpha_{3}$ são imaginários puros. Estimativas dos parâmetros $\alpha$ podem ser feitos a partir de dados experimentais ou de cálculos de primeiros princípios. Nesse trabalho fizemos uma estimativa desses parâmetros a partir de cálculos de primeiros princípios para o sistema físico formado pela interface $\mathrm{Bi}_{2} \mathrm{Se}_{3} /$ GaAs. Na Fig. 4.3 são mostradas estruturas de bandas em casos em que os vínculos das equações (4.9) são violados.

\subsection{Cálculos de primeiros princípios}

Para entender como a interação entre um isolante topológico e um semicondutor é modulada, calculamos as propriedades eletrônicas e texturas de spin da interface de $\mathrm{Bi}_{2} \mathrm{Se}_{3} / \mathrm{GaAs}$ tratadas com Se.

Foram realizados cálculos de primeiros princípios baseados na Teoria do Funcional da Densidade [42,43] como implementado no código Vienna Ab Initio Simulation Package (VASP) [44, 45]. Foram utilizados energias de cortes de $300 \mathrm{eV}$ e amostragens de pontos$k$ de $3 \times 3 \times 1$ no esquema de Monkhorst-Pack [48]. O potencial externo foi calculado usando o método PAW [49,50]. O funcional de troca-correlação utilizado foi na aproximação local da densidade (LDA) como descrito nas referências [46,47]. Também foi incluído o acoplamento spin-órbita para os cálculos das propriedades eletrônicas. 


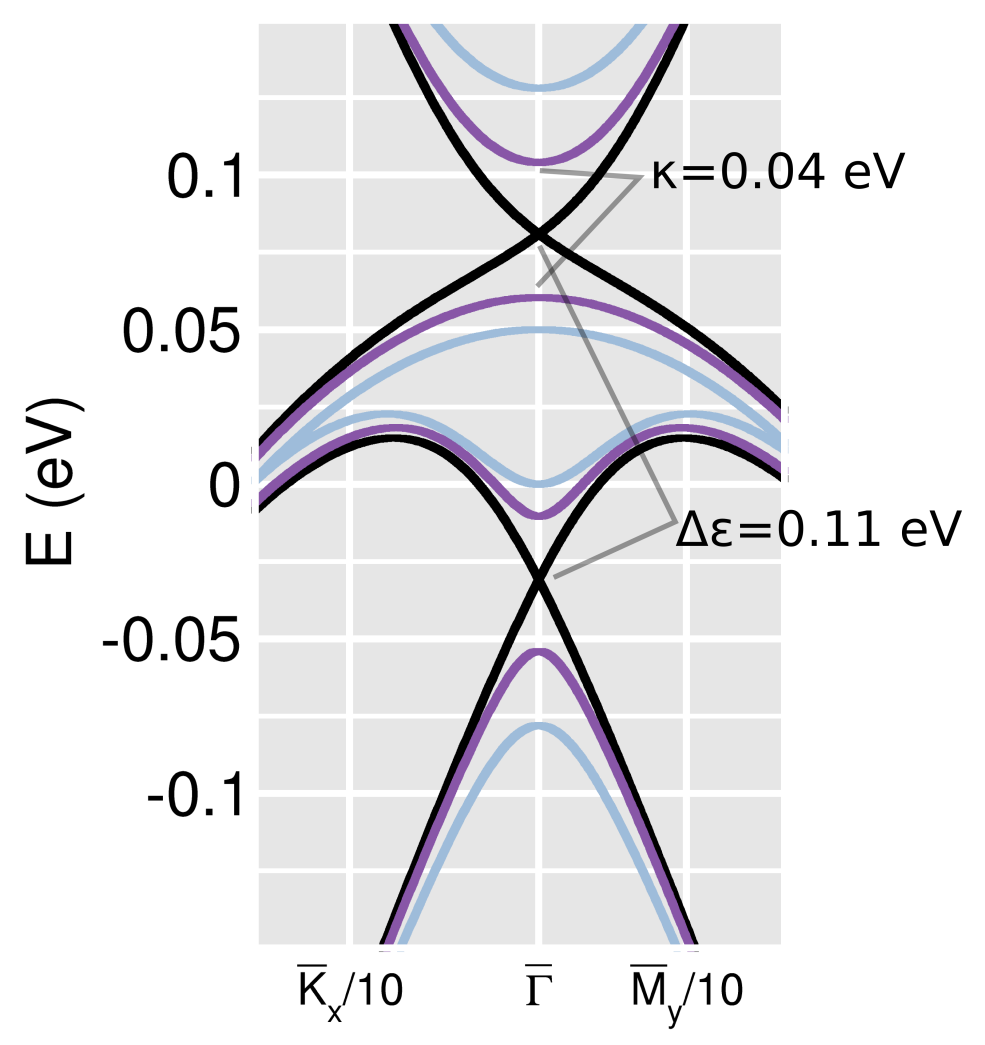

Figura 4.3: Estrutura de bandas para $\alpha_{0}=0.05 \mathrm{eV}$, e $\alpha_{3}=0$ (preto), $\alpha_{3}=0.025 \mathrm{eV}$ (violeta escuro), $\alpha_{3}=0.05 \mathrm{eV}$ (violeta claro). Nos casos $\alpha_{3}>0$ a simetria de reversão temporal é quebrada, desdobrando consequentemente as degenerescências de Kramers. 
A estabilidade química da interface depende do tratamento de Se que é dado à superfície de GaAs(111) terminada em As. Para estudar essa estabilidade química, nós estudamos primeiro as energias de formações de cada configuração em função dos potenciais químicos do Se e do As. Esses cálculos de energias de formações foram realizados na ausência do isolante topológico. Para simular essas superfícies, nós estudamos um modelo de camadas com terminação de Ga passivada com $\mathrm{H}$ com carga fracionária $(q=1.25 e)$ para torna-lá inativa. As terminações de As da superfície GaAs(111) foram estudadas em uma variedade de tratamentos, com átomos substitucionais $\mathrm{Se}_{\mathrm{As}}$ como agentes passivantes cobrindo até uma monocamada de Se (ML, acrônimo do termo em inglês Monolayer). Para uma supercélula $2 \times 2$, nós observamos os tratamentos de Se: 0.00, 0.25 ML, 0.50 ML, 0.75 ML e 1.00 ML. As representações esquemáticas das geometrias do GaAs(111) com tratamentos $0.75 \mathrm{ML}$ e 1.00 ML são mostradas na Fig. 4.4.
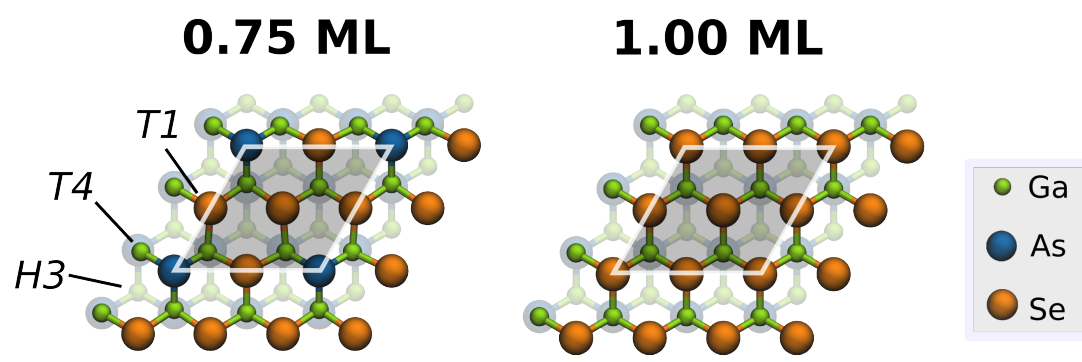

Figura 4.4: Visão superior dos modelos das representações esquemáticas das geometrias das superfícies $0.75 \mathrm{ML}$ (painél à esquerda) e 1.00 ML (painél à direita). As células unitárias dessas superfícies são hachuradas em cinza. Os sítios $H 3, T 4$ e $T 1$ para deposição da primeira camada atômica do isolante topológico são indicados no painel à esquerda.

O mapa de estabilidade química dessas superfícies em função dos potenciais químicos $\mu_{\mathrm{Se}}$ e $\mu_{\mathrm{As}}$ é mostrado na Fig. 4.5. A pas- 
sivação que minimiza a energia total é mostrada em função dos potenciais químicos. Esses potenciais químicos nos limites metálicos são ajustados no zero $\left(\mu_{\mathrm{Se}}^{0}=0\right.$ e $\left.\mu_{\mathrm{As}}^{0}=0\right)$. Já que o processo de crescimento epitaxial deve ocorrer próximo ao limite metálico, apenas os tratamentos $0.75 \mathrm{ML}$ e $1.00 \mathrm{ML}$ vão se estabilizar nesse crescimento. A quase-degenerescência dos tratamentos $0.75 \mathrm{ML}$ e $1.00 \mathrm{ML}$ podem resultar em coexistências dessas fases na interface $\mathrm{Bi}_{2} \mathrm{Se}_{3} / \mathrm{GaAs}$.

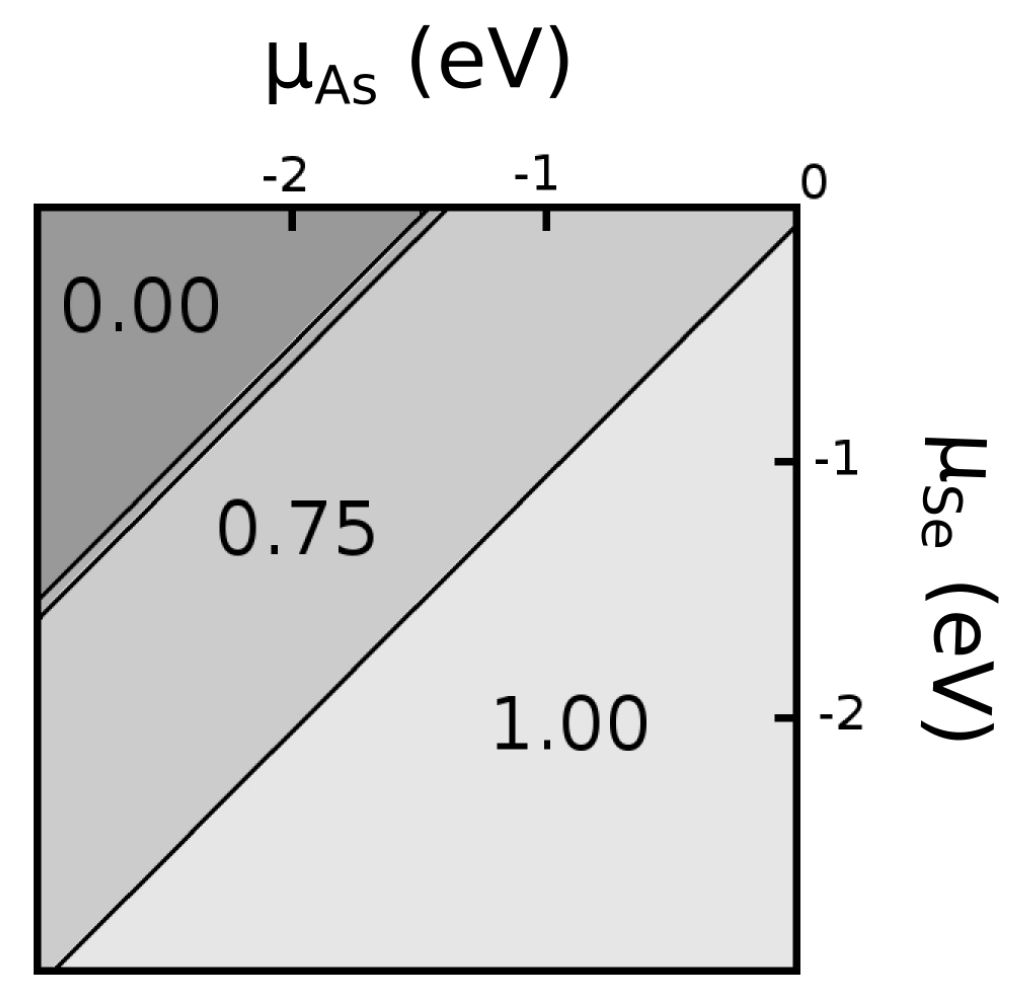

Figura 4.5: Mapa de estabilidade química dos tratamentos de Se das superfícies GaAs(111). São indicadas os tratamentos de Se que minimizam a energia total em função dos potenciais químicos $\mu_{\mathrm{As}}$ e $\mu_{\mathrm{Se}}$. Os potenciais químicos são relativos aos potenciais químicos dos materiais metálicos (Arsênio metal e Selênio metal).

Enquanto que o tratamento $0.75 \mathrm{ML}$ passiva completamente a superfície, resultado em estados de superfícies semicondutores, o tratamento 1.00 ML possui uma sobre-passivação de elétrons que 
resulta em uma fase metálica. A natureza metálica ou semicondutora dos tratamentos de Se na superfície GaAs(111) pode ser explicada através de uma contagem de elétrons. No GaAs, se considerarmos que cada elétrons $4 s$ e $4 p$ do Ga e As participam das ligações químicas, os átomos de Ga contribuiriam com três elétrons, enquanto que os átomos de As contribuiriam com cinco elétrons nessas ligações. Cada átomo de Ga possui $\frac{3}{4}$ elétrons por ligação, e cada átomos de As possui $\frac{5}{4}$ elétrons por ligação. Como a superfície de GaAs terminada em As deixam quatro átomos de As pendentes, há uma falta de quatro elétrons para cada átomo de Ga faltando, ou seja, três buracos numa supercélula $2 \times 2$ de GaAs(111). Já que o Se possui seis elétrons, ele age como um doador de elétron quando substitui um átomo de $\mathrm{As}\left(\mathrm{Se}_{\mathrm{As}}\right)$, com esse elétron extra aniquilando um dos buracos deixados pela falta do Ga. Para os tratamentos $0.25 \mathrm{ML}$ e $0.50 \mathrm{ML}$, as superfícies tem dois e um buracos. No tratamento $0.75 \mathrm{ML}$ todos os buracos são eliminados, deixando a superfície semicondutora. Com um elétron adicional do caso 1.00 ML, a superfície torna-se metálica novamente.

As estruturas de bandas das superfícies de GaAs com os tratamentos 0.75 ML e 1.00 ML são mostradas na Fig. 4.6. Enquanto que no caso $0.75 \mathrm{ML}$ a estrutura de bandas da superfície é semicondutora, como esperado pela contagem de elétrons, o caso 1.00 ML apresenta uma estrutura de bandas metálicas. Esses estados metálicos são localizados na região da superfície devido à sobrepassivação de Se nas terminações de As.

Sobre a superfície GaAs com tratamento de $0.75 \mathrm{ML}$, nós empilhamos $6 \mathrm{QL}$ de $\mathrm{Bi}_{2} \mathrm{Se}_{3}$ para criar uma interface. Devido à discordância de $3,5 \%$ entre os parâmetros de rede do $\mathrm{Bi}_{2} \mathrm{Se}_{3}(4,138 \AA ⿱ ⺈)$ e do GaAs (3,997 Å), utilizamos uma supercélula $2 \times 2$ com um parâmetro de rede intermediário (4,068 Å) e 1,7\% de discordância de parâmetro de rede.

Os empilhamentos de $\mathrm{Bi}_{2} \mathrm{Se}_{3}$ foram calculados em três configurações diferentes, com a primeira camada atômica de Se acima 


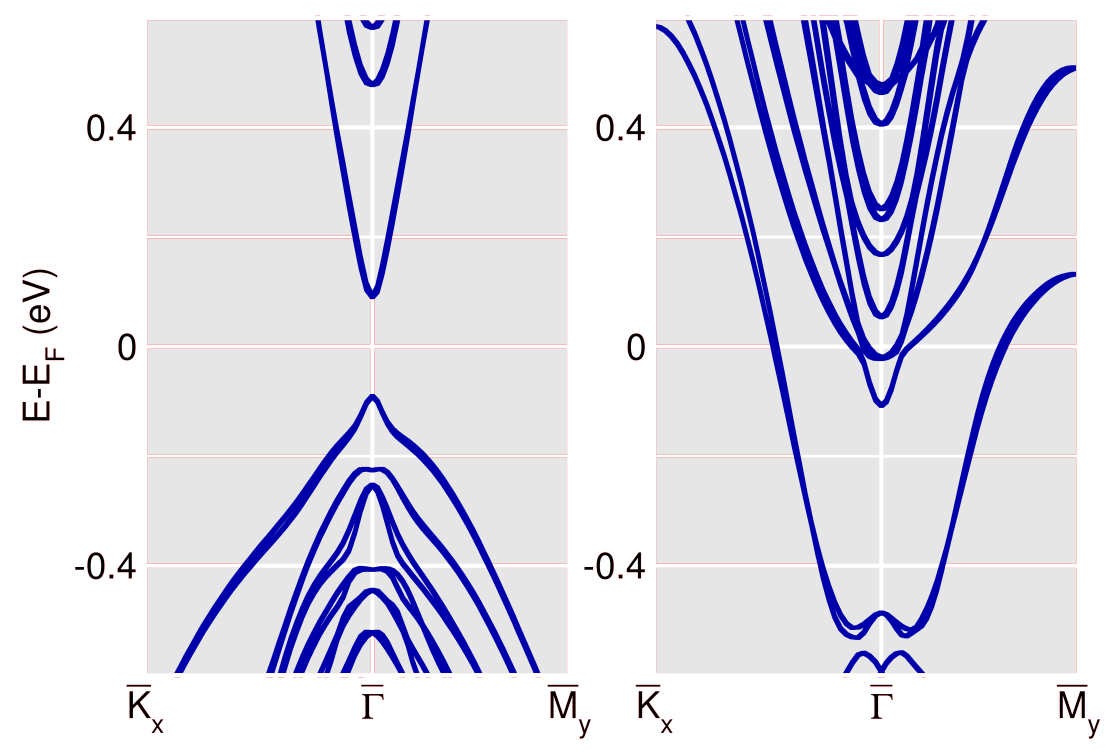

Figura 4.6: Estruturas de bandas das superfícies de GaAs com tratamentos: 0.75 ML (painel à esquerda), 1.00 ML (painel à direita). Para superfícies GaAs com tratamentos de Se com 0.75 ML, o material é isolante com gap de energia em torno de $0.2 \mathrm{eV}$. Para superfícies com tratamento de Se de $1.00 \mathrm{ML}$, o material é metálico. 
dos sítios: H3, T4 e T1, como mostrado na Fig. 4.4. A energia de formação para a configuração $H 3$ foi a menor encontrada, com as configurações $T 4$ e $T 1$ com energias $0,022 \mathrm{eV}$ e $0,493 \mathrm{eV}$ acima do H3.

No intuito de estudar a estrutura de bandas da interface entre $\mathrm{Bi}_{2} \mathrm{Se}_{3}$ e GaAs, nós aplicamos o método de bandas projetadas na área sombreada da Fig. 4.7(a) (duas camadas atômicas do $\mathrm{Bi}_{2} \mathrm{Se}_{3}$ e duas camadas atômicas do GaAs.) As funções de onda foram projetadas nos harmônicos esféricos reais centralizados em cada átomo dentro da região projetada. Apenas funções de onda dentro da esfera de Wigner-Seitz do método PAW foram consideradas nessas projeções.

Levando em consideração que as projeções estão em regiões de porcentagens críticas de $5 \%-15 \%$ e maiores de $15 \%$, nós obtemos as estruturas de bandas da Fig. 4.7(b). Baseados nesses resultados, notamos que os estados de interface foram majoritariamente projetados em quatro bandas próximas ao nível de Fermi (para 0.75 $\mathrm{ML}$ ), e em algumas bandas entre $0,1 \mathrm{e} 0,6 \mathrm{eV}$ abaixo do nível de Fermi. Para identificar os estados de interface vindo da topologia de bandas não-trivial, nós vamos nos focar na estrutura de bandas e texturas de spin para quatro bandas próximas ao ponto $\bar{\Gamma}$.

\subsection{Interação TI/S}

Para analisar a manifestação da interação entre o isolante topológico e o semicondutor, nós comparamos a estrutura de bandas e texturas de spin na vizinhança de $\bar{\Gamma}$. Na Fig. 4.8 são mostradas as estruturas de bandas calculadas usando métodos de primeiros princípios para $0.75 \mathrm{ML}$. São destacadas quatro bandas $(b 1, b 2, b 3$, e b4) que podem ser comparadas diretamente com os estados do modelo Hamiltoniano efetivo da equação 4.2.

Para reproduzir a banda de primeiros princípios, nós compara- 


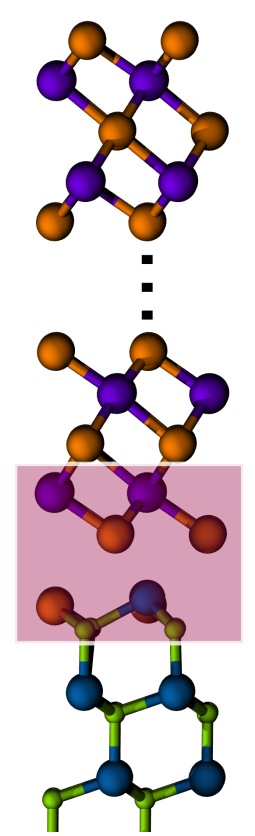

(a)

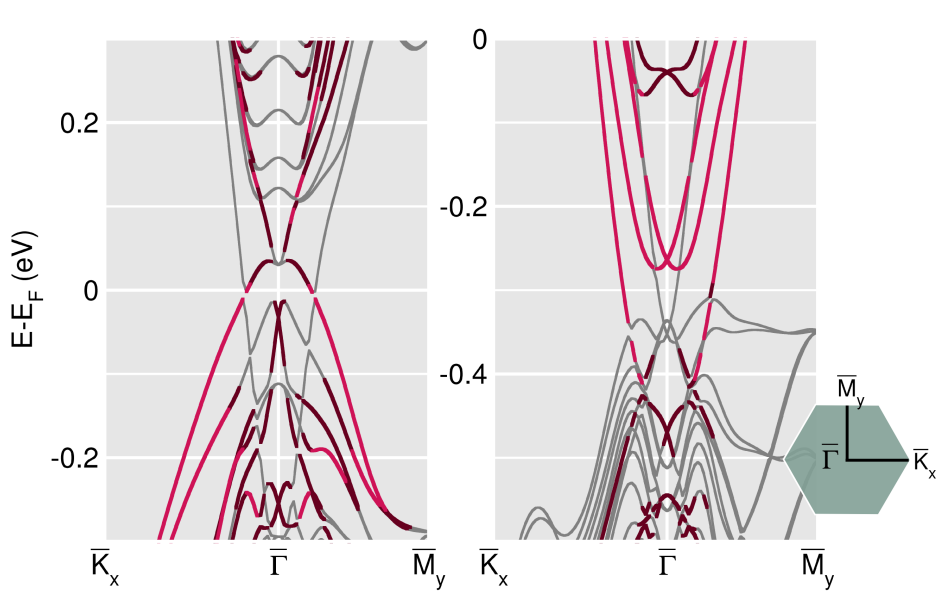

(b)

Figura 4.7: (a) Representação esquemática da interface $\mathrm{Bi}_{2} \mathrm{Se}_{3} / \mathrm{GaAs}$ com tratamento de Se de 0.75 ML, e empilhada no sítio H3. A representação espacial da projeção da função de onda é mostrada na região hachurada em vermelho. (b) Estruturas de bandas das interfaces com tratamentos $0.75 \mathrm{ML}$ (esquerda) e 1.00 ML (direita), com projeções críticas de funções de onda de 5\%-15\% (vermelho claro) e $>15 \%$ (vermelho escuro). As estruturas de bandas totais são mostradas em cinza escuro. 

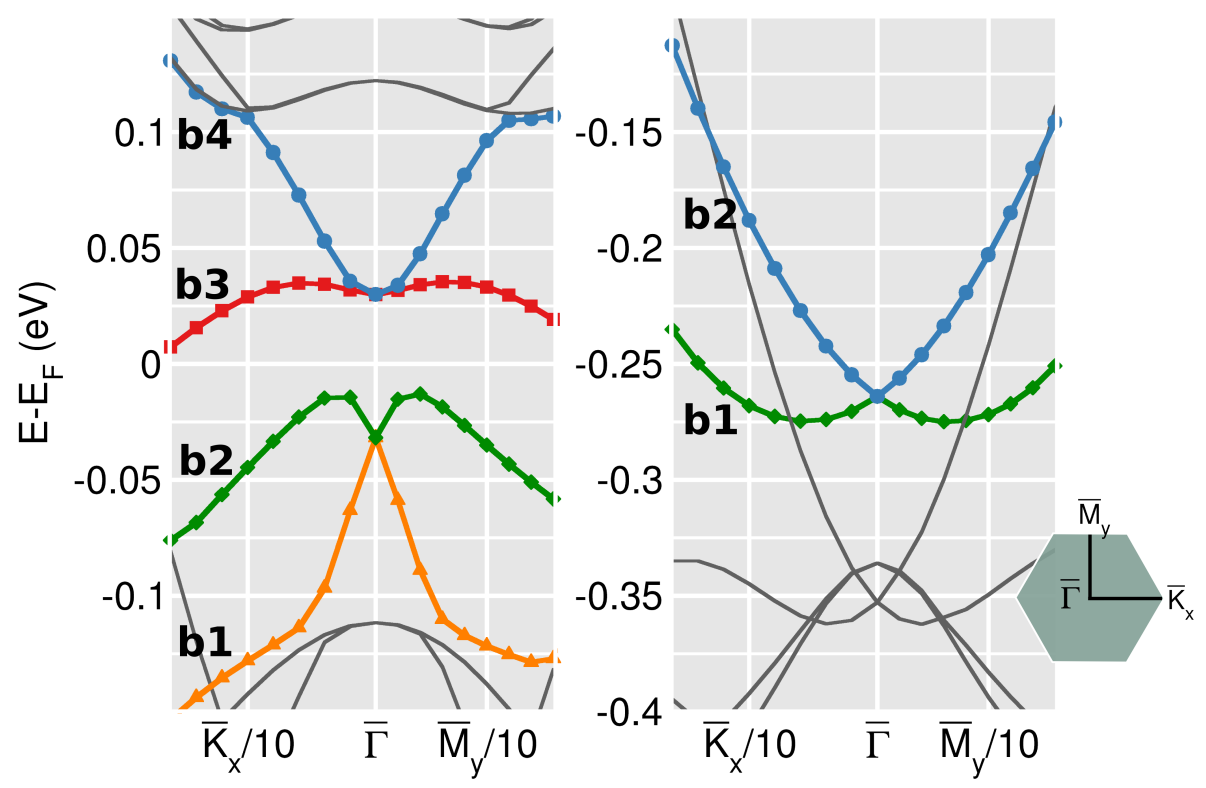

Figura 4.8: Estrutura da bandas calculadas por métodos de primeiros princípios para $0.75 \mathrm{ML}$ (painél à esquerda) e 1.00 ML (painél à direita). Os estados de interface para o caso $0.75 \mathrm{ML}$ : $b 1$ (em amarelo), $b 2$ (em verde), $b 3$ (em vermelho), e $b 4$ (em azul) são rotulados para efeitos de comparações com os resultados obtidos pelo modelo Hamiltoniano efetivo. Os estados de interface $b 1$ (em verde), e $b 2$ (em azul) do caso $1.00 \mathrm{ML}$ também são apresentados na estrutura de bandas. 
mos cada caso onde pelo menos um dos parâmetros $\alpha$ é diferente de zero. Nós encontramos que as estruturas de bandas para $\alpha_{0}=0.05$ $\mathrm{eV}$ e $\alpha_{1}=\alpha_{2}=\alpha_{3}=0$; e $\alpha_{3}=i 0.05 \mathrm{eV}, \alpha_{1}=\alpha_{2}=\alpha_{3}=0$ são as mesmas. A estrutura de bandas para o último caso é mostrado na Fig. 4.9. A estrutura de bandas para $\alpha_{1}=i 0.05 \mathrm{eV}, \alpha_{0}=\alpha_{2}=\alpha_{3}=0$; e $\alpha_{2}=i 0.05 \mathrm{eV}, \alpha_{0}=\alpha_{1}=\alpha_{3}=0$ também são as mesmas da Fig. 4.9.

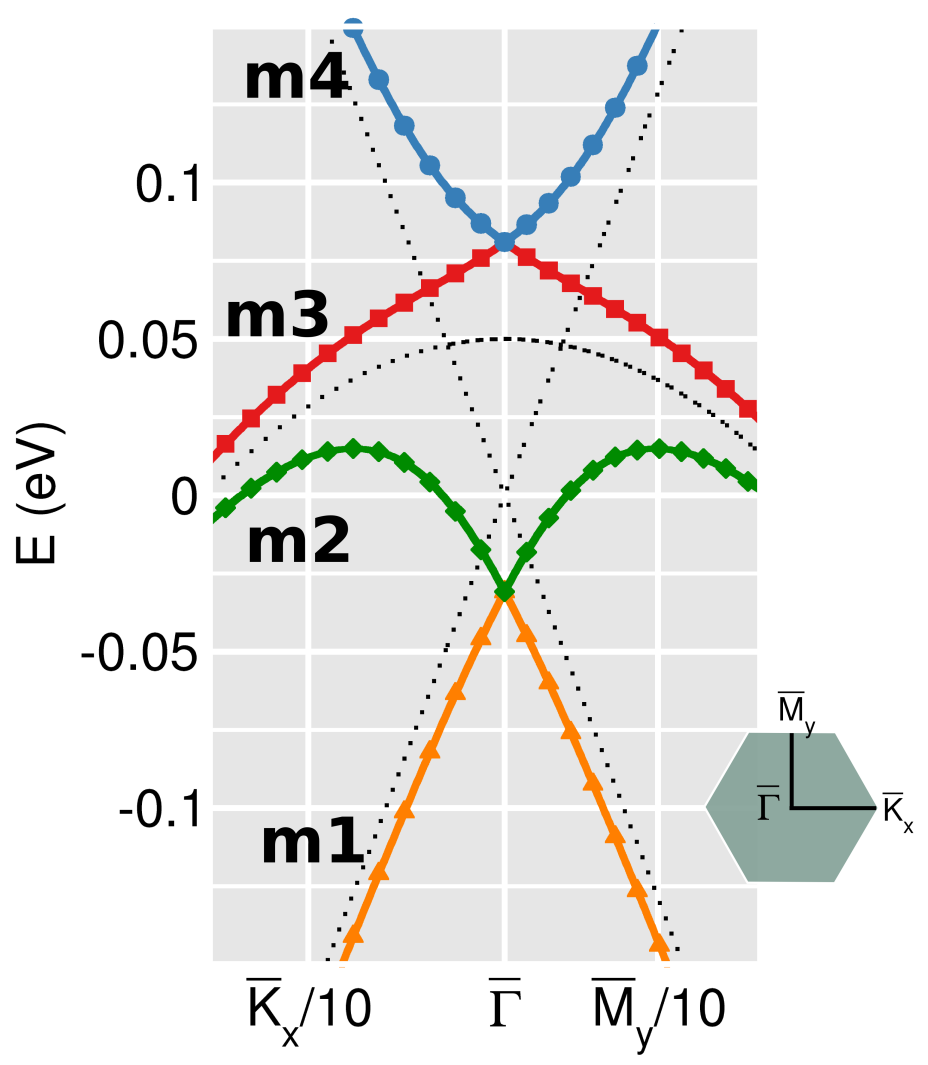

Figura 4.9: Estrutura da bandas do modelo Hamiltoniano $\operatorname{com} \alpha_{3}=$ $i 0.05 \mathrm{eV}$, e $\alpha_{0}=\alpha_{1}=\alpha_{2}=0$. Outros parâmetros utilizados foram: $v_{F}=5 \cdot 10^{5} \mathrm{~m} / \mathrm{s}, \Delta=0.05 \mathrm{eV}$, e $m_{*}=-0.456 m_{0}$. Os estados $m 1$ (em amarelo), $m 2$ (em verde), $m 3$ (em vermelho), e $m 4$ (em azul) são comparáveis com os estados $b 1, b 2, b 3$ e $b 4$ dos cálculos em primeiros princípios.

Embora essas estruturas de bandas sejam idênticas, as texturas de spin são distintas. Enquanto que os casos $\alpha_{0} \neq 0$ ou $\alpha_{3} \neq 0$ 
tem texturas de spin helicoidais para os estados $m 1, m 2, m 3$, e $m 4$, os casos $\alpha_{1} \neq 0$ ou $\alpha_{2} \neq 0$ tem texturas de spin não-helicoidais para os estados $m 2$ e $m 3$. Os estados $m 1$ e $m 4$ são helicoidais mesmo para $\alpha_{1} \neq 0$ ou $\alpha_{2} \neq 0$. Finalmente nós mostramos que $\alpha_{3}=i 0.05 \mathrm{eV}$ e $\alpha_{0}=\alpha_{1}=\alpha_{2}=0$ fornecem texturas de spin compativeis com os obtidos a partir de cálculos de primeiros princípios.
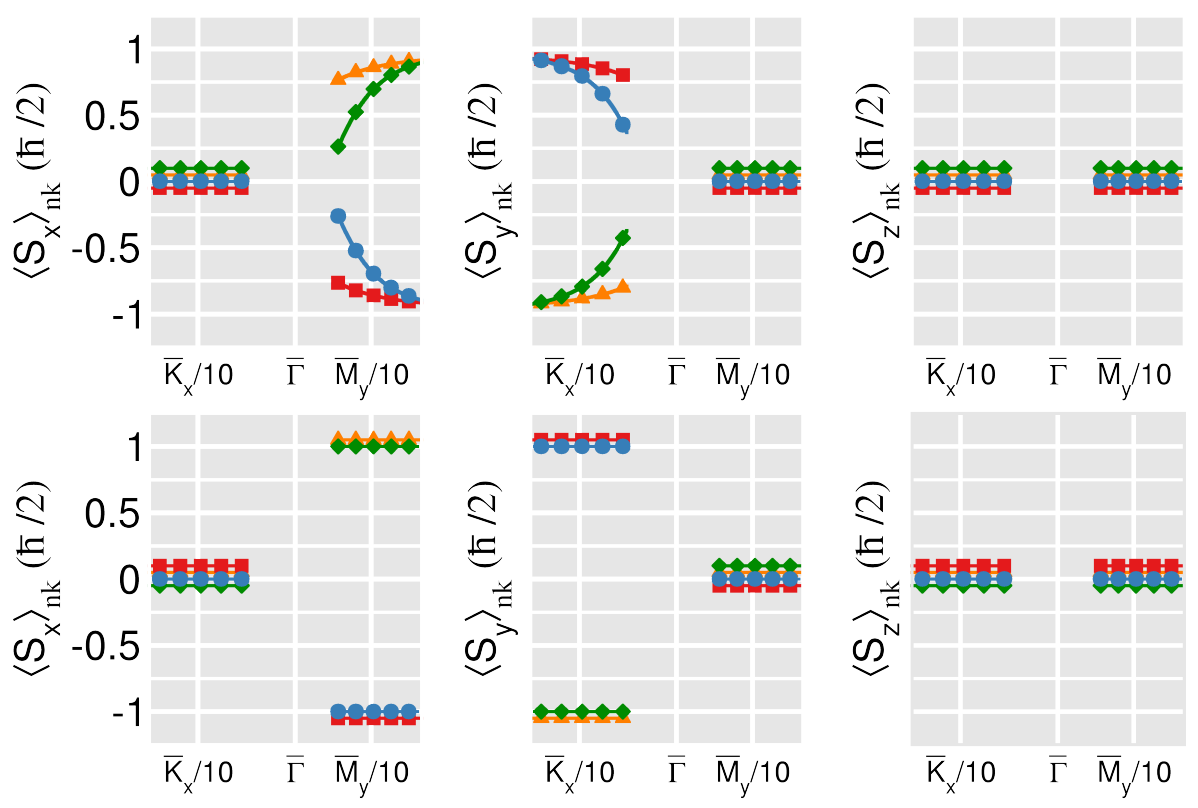

Figura 4.10: Texturas de spin para o modelo Hamiltoniano para: $\alpha_{3}=i 0.05 \mathrm{eV}, \alpha_{0}=\alpha_{1}=\alpha_{2}=0$ (painéis superiores), e $\alpha_{0}=0.05 \mathrm{eV}$, $\alpha_{1}=\alpha_{2}=\alpha_{3}=0$ (painéis inferiores). As colorações de cada projeção são relacionadas aos estados $m 1$ (em amarelo), $m 2$ (em verde), $m 3$ (em vermelho), e $m 4$ (em azul) para o modelo Hamiltoniano efetivo.

As texturas de spin calculadas via métodos de primeiros princípios para os estados $b 2$ e $b 4$ sugerem que nós temos um desdobramento no cone de Dirac em $\bar{\Gamma}$, como mostrado na Fig. 4.9. O desdobramento de energia em $\bar{\Gamma}$ e a textura de spin para $m 2$ e $m 4$ são obtidos para interações parametrizadas por $\alpha_{3}=i 0.05 \mathrm{eV}$, $\alpha_{0}=\alpha_{1}=\alpha_{2}=0$, como mostrado na Fig. 4.10. As texturas de spin para os estados $b 2$ e $b 4$ na Fig. 4.10 e os estados $m 2$ e $m 4$ na 
Fig. 4.11 são qualitativamente iguais, indicando mesmo o desdobramento do cone de Dirac com simetria de reversão temporal.
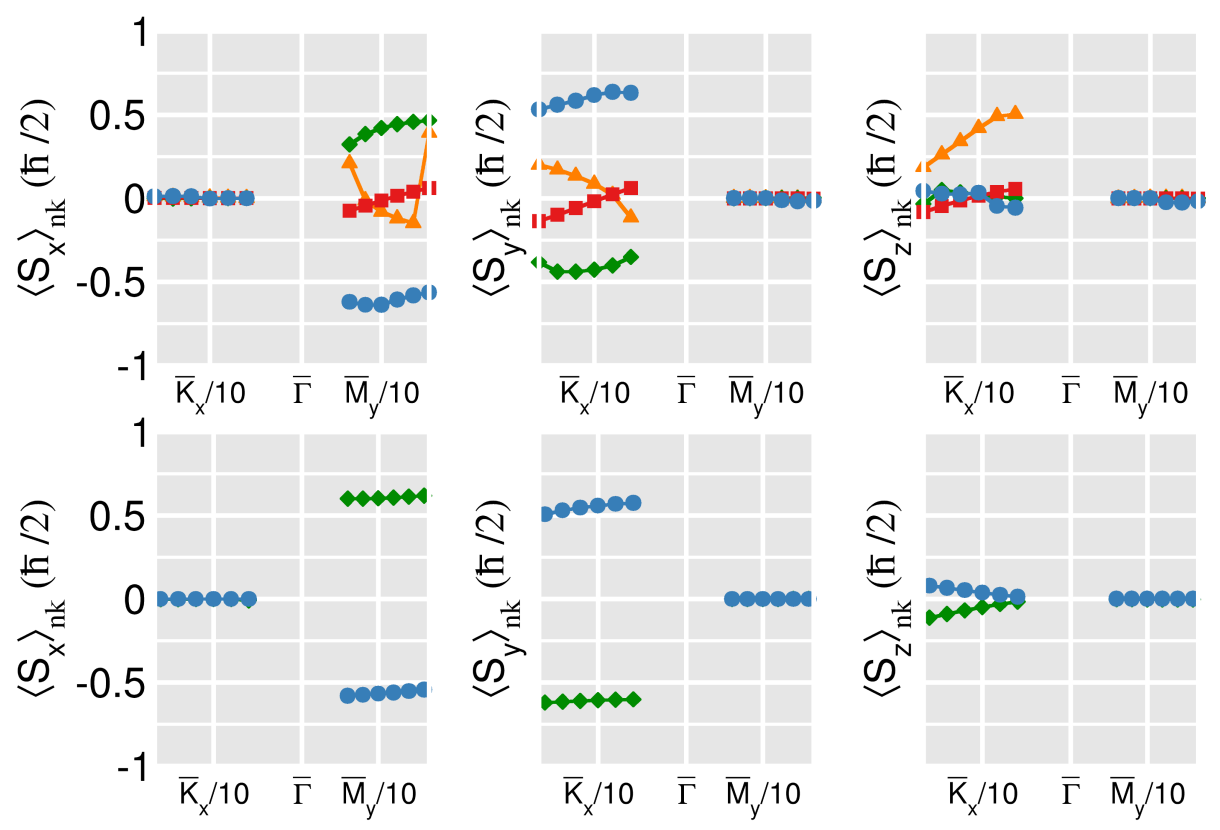

Figura 4.11: Texturas de spin calculada a partir de métodos de primeiros princípios para: $0.75 \mathrm{ML}$ (painéis superiores), e $1.00 \mathrm{ML}$ (painéis inferiores). No caso $0.75 \mathrm{ML}$, as colorações de cada projeção estão relacionadas aos estados $b 1$ (em amarelo), $b 2$ (em verde), $b 3$ (em vermelho), e $b 4$ (em azul). No caso $1.00 \mathrm{ML}$, são mostradas as projeções de spin para os estados b1 (em verde), e b2 (em azul).

As interações entre os isolantes topológicos e os semicondutores parametrizados pelos parâmetros $\alpha$ indicam que nós podemos obter um desdobramento do cone de Dirac em $\bar{\Gamma}$ sem mudar a topologia de bandas de bulk. Enquanto que o cone de Dirac pode ser quebrado por formações de ligas ou dopanges magnéticas, a interação entre bandas em interfaces do tipo-II ou tipo-III mantém a simetria de reversão temporal e consequentemente as degenerescências de Kramers em $\bar{\Gamma}$. 


\subsection{Conclusão}

As interações entre os cone de Dirac de um isolante topológico e a banda de valência (ou banda de condução) de um semicondutor em uma interface do tipo-II ou tipo-III pode levar à um desdobramento de energia no cone de Dirac. Embora haja gap de energia no ponto $\bar{\Gamma}$, as degenerescências de Kramers e texturas de spin helicoidais permanecem quase inalteradas. Esses resultados foram estudados por um modelo Hamiltoniano efetivo e por cálculos de primeiros princípios baseados na DFT sem parâmetros empíricos. Embora nossos cálculos de primeiros princípios sejam realizados para a interface $\mathrm{Bi}_{2} \mathrm{Se}_{3}(0001) / \mathrm{GaAs}(111)$, nós argumentamos que nosso modelo pode ser expandido para outras interfaces do tipo-II ou tipo-III entre isolantes topológicos e semicondutores. O fenômeno emergente de desdobramento do cone de Dirac com simetria de reversão temporal podem nos guiar no desenvolvimento de dispositivos spintrônicos baseados em TI/S, especialmente devido aos estados topológicos helicoidais e seu papel na geração de correntes de spin [22]. Essas interfaces também devem se beneficiar dos comprimentos de coerência de spin obtidos em semicondutores como o GaAs. 
68 Interfaces entre isolantes topológicos e semicondutores 


\section{Nanoestradas de germaneno}

What I cannot create, I do not understand.

Richard Feynman

\subsection{Introdução}

Como mencionado no Capítulo 1, a ideia de efeito Hall quântico de spin (QSHE, acrônimo do termo em inglês surgiu do estudo do acoplamento spin-órbita no grafeno [12,37]. Devido ao pequeno gap de energia vindo do SOC, essa ideia perdeu o interesse com o tempo. O gap de energia calculado por métodos de primeiros princípios [67] foi da ordem de poucos $\mu \mathrm{eV}$.

Outros materiais 2D foram propostos depois de 2005 para se observar o QSHE, entre alguns desses materiais estão: siliceno [38, 39, 68, 69], germaneno [40], estaneno [70], e ligas hexagonais 2D desordenadas $\mathrm{Si}_{x} \mathrm{Ge}_{1-x}$ [71] (ver Capítulo 6). Esses materiais apresentaram geometrias semelhantes ao grafeno, mas com as seguintes alterações: (i) a rede hexagonal possui uma distorção vertical entre sub-redes, mostrado na Fig. 5.1; (ii) o acoplamento spin-órbita nesses materiais são relativamente grandes compara- 
dos com o do grafeno. Os gaps de energias nesses materiais são da ordem de meV; e (iii) o invariante topológico $\mathbf{Z}_{2}[40,71]$ para esses materiais é não-trivial. Acoplamento spin-órbita "grande" (em comparação ao do grafeno) e não-trivialidade de bandas são características essenciais para a observação do QSHE em condições experimentais realistas.

Do ponto de vista experimental, a busca por isolantes topológicos foi intensificada pela sintetização do siliceno. O siliceno é geralmente crescido em superfícies metálicas de prata [38, 68, 69]. Esses substratos metálicos possuem orbitais $d$ que hidridizam com os orbitais $p$ do siliceno, destruindo as propriedades topológicas do material. Além disso, medições de correntes nesses sistemas introduzem dificuldades em separar contribuições do isolante topológico das contribuições do substrato metálico.

Outro problema relacionado ao siliceno é que seu gap de energia devido ao SOC é de apenas 1, $9 \mathrm{meV}$, o que limitaria a observação do efeito Hall quântico de spin à temperaturas extremanente baixas.

\subsection{O germaneno e o germanano}

O germaneno é um material 2D formado por átomos de germânio em uma rede hexagonal com distorção vertical em uma das sub-redes. Assim como o siliceno [40], o germaneno também é um isolante topológico $2 \mathrm{D}$ com invariante topológico não-trivial $\mathbf{Z}_{\mathbf{2}}=1$. O gap de energia devido ao acoplamento spin-órbita é de $\Delta_{g}=25$ meV. Esse gap de energia relativamente grande permitiria observar o efeito Hall quântico de spin em temperaturas acessíveis.

Semelhantemente ao germaneno, o germanano é um material 2D formado por átomos de germânio e hidrogênio. Esse material pode ser entendido como um germaneno completamente hidrogenado, como mostrado na Fig. 5.1(a). Diferente do germaneno, o 
(a)

\section{Germanene}

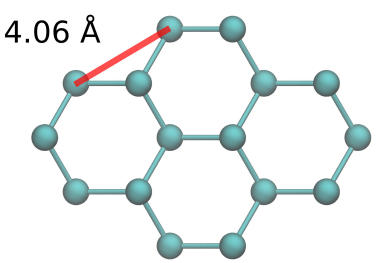

$0.69 \AA$

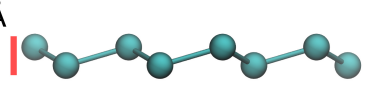

Hydrogenated Germanene (Germanane)

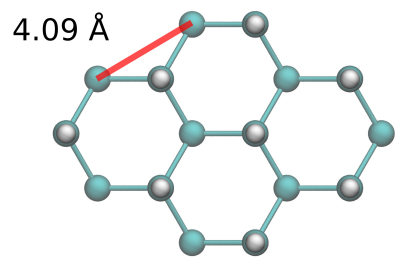

$0.74 \AA$

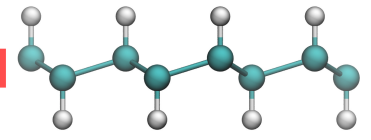

(b)

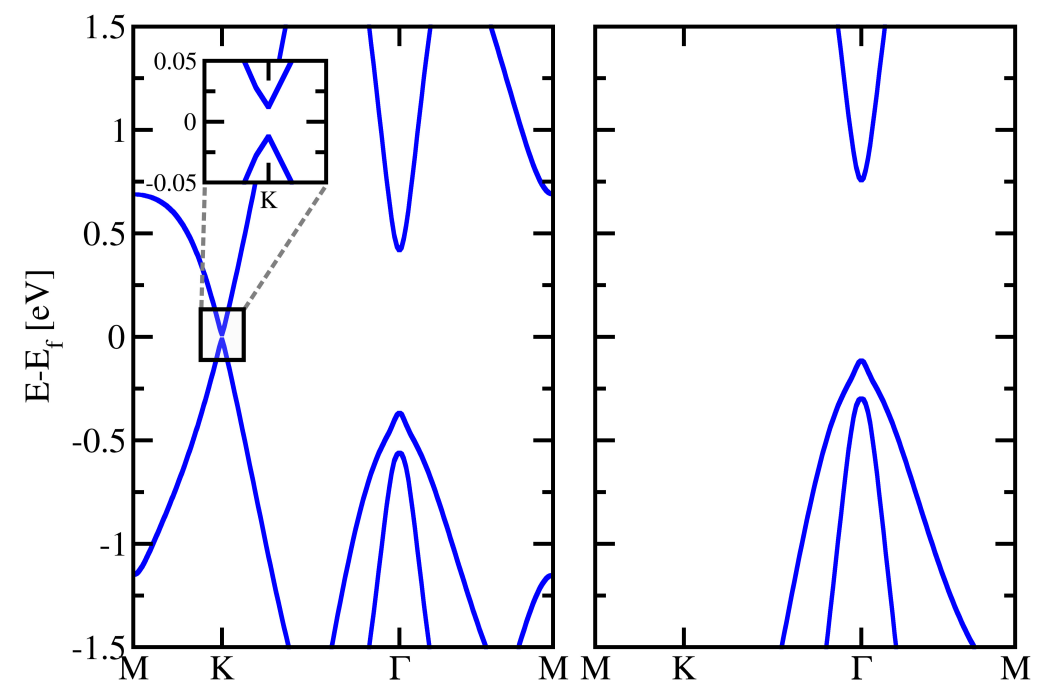

Figura 5.1: (a) Representações esquemáticas do germaneno (Ge) e do germanano $(\mathrm{GeH})$. São comparadas as constantes de rede desses materiais e as distâncias das distorções verticais entre subredes. No germanano, a constante de rede é $0.03 \AA ̊$ maior do que a do germaneno, e a distorção vertical entre sub-rede é maior $0.05 \AA$ em relação ao germaneno. (b) Estrutura de bandas do germaneno (painel à esquerda) e do germanano (painel à direita). Para a estrutura de bandas do germaneno, é também mostrada uma ampliação da estrutura de bandas em torno do ponto $K$. 
germanano é um isolante trivial com gap de energia de $0,87 \mathrm{eV}^{1} \mathrm{e}$ $Z_{2}=0$. A estrutura de bandas desse material foi calculada através de métodos de primeiros princípios e é mostrada na Fig. 5.1(b). O material foi sintetizado em 2013 por Bianco et al. através da deintercalação topoquímica do $\mathrm{CaGe}_{2}$ [72], como mostrado na Fig. 5.2. A obtenção de camadas simples de germanano foi obtida através de esfoliação mecânica das multicamadas de germanano.

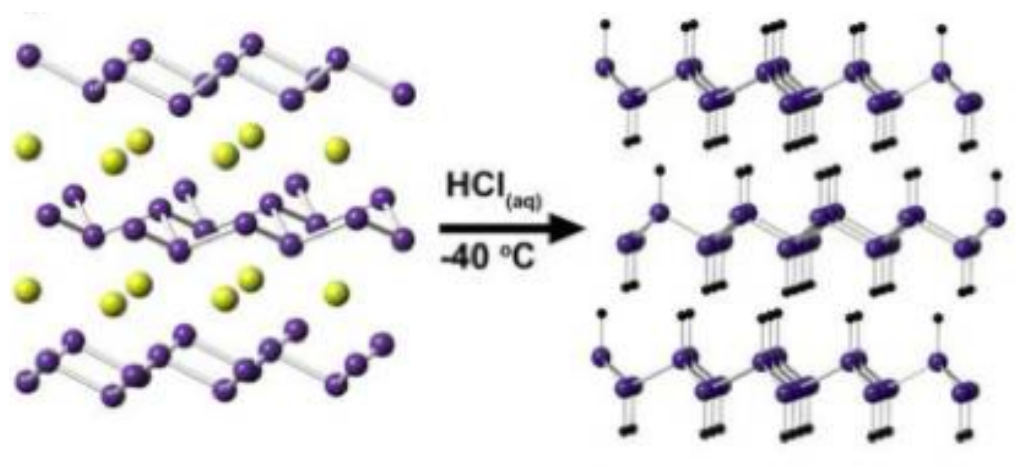

Figura 5.2: Representação esquemática do processo de formação das multicamadas de germanano. A aplicação do ácido $\mathrm{HCl}$ no $\mathrm{CaGe}_{2}$ remove os átomos de $\mathrm{Ca}$ (em amarelo) e funcionaliza as camadas de Ge (em roxo) com átomos de $\mathrm{H}$ (em preto). Figura extraída da referência [72].

Através da formação de nanoestradas de germaneno imersas em germanano, proporemos um arranjo experimental para a obtenção do QSHE. Essas nanoestradas de germaneno são formadas pela dissociação de hidrogênio via corrosão por plasma em uma região na forma de uma nanofita. Esse tipo de nanoestruturas são semelhantes as construídas por nanoestradas de grafeno imersas em grafano [73, 74], ilustrados na Fig. 5.3.

Entretanto, já que o germaneno é um isolante topológico 2D e o germanano é um isolante trivial, os estados de interfaces entre esses materiais são estados metálicos topologicamente protegidos por perturbações com simetria de reversão temporal. A estabilidade

\footnotetext{
${ }^{1} \mathrm{O}$ gap de energia experimental observado é de $1.53 \mathrm{eV}$.
} 
a)

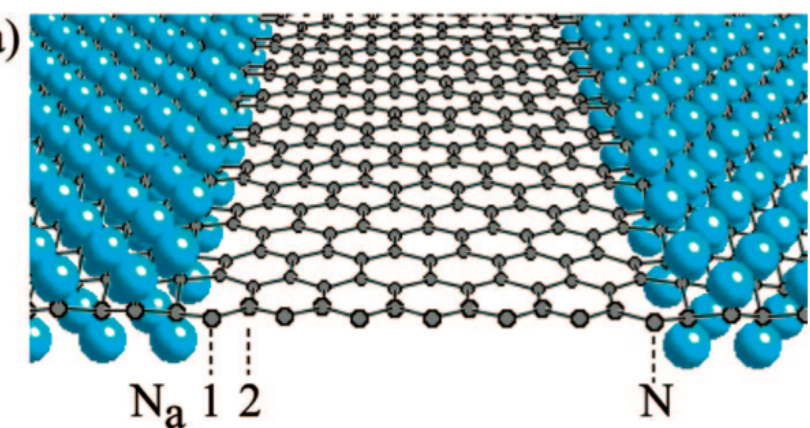

b)

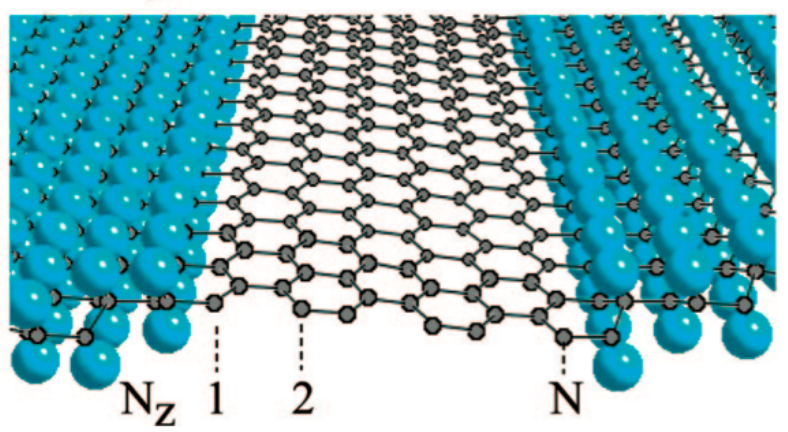

Figura 5.3: Representação esquemática das nanoestradas de grafeno imersas em grafano: (a) interface armchair, (b) interface zigzag. Figura extraída da referência [73]. 
química e propriedades eletrônicas desses sistemas foram calculadas através de métodos de primeiros princípios baseados na DFT (ver apêndice $\mathrm{A}$ ).

Nesse trabalho os cálculos de primeiros princípios foram realizados através dos códigos OpEnMX [75] para as estruturas de bandas, e VASP $[44,45]$ para as texturas de spin e energias totais. Foram utilizados funcionais de troca-correlação GGA-PBE [76], e acoplamentos spin-órbitas com polarizações de spin não-colineares. Todos as geometrias estudadas foram relaxadas para forças residuais menores que $0,001 \mathrm{eV} / \AA$. . As texturas de spin foram calculadas para estados de interfaces pelo vetor

$$
P_{n}(\mathbf{k})=\left\langle u_{n \mathbf{k}}^{\sigma}|\sigma| u_{n \mathbf{k}}^{\sigma}\right\rangle,
$$

onde $\left|u_{n \mathbf{k}}^{\sigma}\right\rangle$ são os estados de Bloch das interfaces e $\boldsymbol{\sigma}=\left(\sigma_{x}, \sigma_{y}, \sigma_{z}\right)$ é o vetor de matrizes de Pauli.

A Fig. 5.1(a) mostram as geometrias do germaneno e do germanano. A distorção vertical entre sub-redes é aumentada em 0,05 Å no germanano. Na Fig. 5.1(b) são mostradas as estruturas de bandas do germaneno (esquerda) e do germanano (direita). $\mathrm{O}$ germaneno apresenta um gap de energia de $0,025 \mathrm{eV}$ no ponto $K$ devido ao acoplamento spin-órbita, enquanto que o germanano possui gap de energia de $0,87 \mathrm{eV}$ no ponto $\Gamma$.

O germaneno é um isolante topológico com $Z_{2}=1$, enquanto que o germanano é um isolante trivial, $Z_{2}=0$. Uma interface entre esses dois materiais deve apresentar estados metálicos topologicalmente protegidos por simetria de reversão temporal. Nesse trabalho é proposto que a formação de nanoestradas de germaneno em germanano origina estados metálicos de interface topologicamente protegidos. Esses estados de interfaces possuem polarizações de spin que poderiam gerar correntes de spin sem dissipação, com potenciais aplicações em spintrônica. 


\subsection{Dissociação de hidrogênio em germa- nano}

No intuito de estudar a estabilidade química da formação dessas nanoestradas de germaneno, as energias de formação de vacâncias de hidrogênio $\left(V_{H}\right)$ e divacâncias de hidrogênio $\left(D_{H}\right)$ em germanano, e energias de formação de vacâncias de germânio $\left(\mathrm{V}_{\mathrm{Ge}}\right)$ em germaneno (ver Fig. 5.4) foram calculadas a partir de métodos de primeiros princípios. Se a energia de formação das vacâncias de germânio em germaneno forem menores ou aproximadamente iguais as energias de formações das vacâncias e divacâncias de hidrogênio em germanano, a formação das nanoestradas de germaneno por dissociação de hidrogênio em germaneno é comprometida. As energias fornecidas para dissociar hidrogênios em germanano poderiam redistribuir e destruir as nanoestradas de germaneno por formações de vacâncias de germânio. Usando supercélulas $4 \times 4$ de células unitárias, as energias de formações encontradas foram $\Delta H_{f}\left(V_{\mathrm{H}}\right)=1,03 \mathrm{eV}, \Delta H_{f}\left(D_{\mathrm{H}}\right)=0,57 \mathrm{eV}$, e $\Delta H_{f}\left(V_{\mathrm{Ge}}\right)=2,36 \mathrm{eV}$. Esses resultados foram comparados com as energias de formações de vacâncias de carbono em grafeno: $\Delta H_{f}\left(V_{\mathrm{C}}\right)=8,04 \mathrm{eV}$, e as energias de formações de vacâncias e divacâncias de hidrogênio em grafano: $\Delta H_{f}\left(V_{\mathrm{H}}\right)=1,98 \mathrm{eV}, \Delta H_{f}\left(D_{\mathrm{H}}\right)=0,75 \mathrm{eV}$. Desses resultados podemos sugerir que a partir de um experimento com feixe controlado, é possivel formar as nanoestradas de germaneno em germanano.

\subsection{Nanoestradas de germaneno imersas em germanano}

Baseada nessa ideia de dissociação local de hidrogênio, nós montamos geometrias de nanoestradas de germaneno com bordas/interfaces zigzag e armchair, como mostrado na Fig. 5.5(a) e 5.5(b). As propriedades eletrônicas dessas geometrias foram calcu- 
(a)

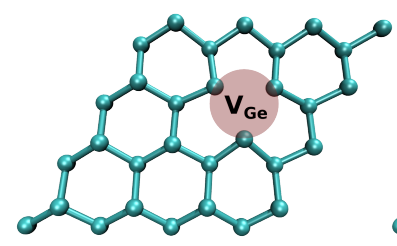

(b)

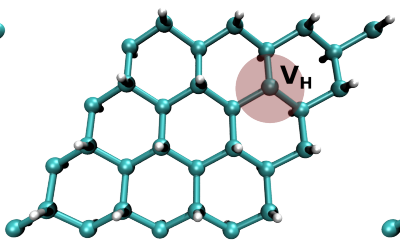

(c)

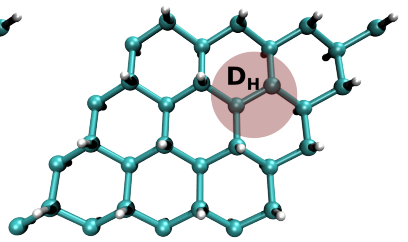

Figura 5.4: Representações esquemáticas das (a) vacâncias de germânio $\left(\mathrm{V}_{\mathrm{Ge}}\right)$ em germaneno, (b) vacâncias de hidrogênio $\left(\mathrm{V}_{\mathrm{H}}\right)$ em germanano, e divacâncias de hidrogênio $\left(\mathrm{D}_{\mathrm{H}}\right)$ em germanano. As regiões de formações dos defeitos são destacadas em vermelho.

ladas em função de várias larguras, rotuladas por $W_{a}$ para bordas armchair e $W_{z}$ para bordas zigzag. A variável $W$ representa o número de hexágonos expostos nas nanoestradas de germaneno. As larguras dessas nanoestradas em função de $W$ (em $\AA$ ) são:

$$
L\left(W_{z}\right)=1,14+3,56 W_{z}
$$

para bordas zigzag, e

$$
L\left(W_{a}\right)=1,93+2,05 W_{a}
$$

para bordas armchair.

Para nanoestradas com bordas armchair, estudamos geometrias com larguras de $W_{a}=1$ até $W_{a}=32$, enquanto que para nanoestradas com bordas zigzag estudamos geometrias de $W_{z}=1$ até $W_{z}=17$. Para nanoestradas zigzag muito estreitas, de $W_{z}=1$ até $W_{z}=7$, todos os sistemas apresentaram gaps de energias e ausências de estados topológicos de interfaces. Esse comportamento para larguras estreitas surgem devido à interação entre as interfaces da nanoestradas. Para larguras maiores que $W_{z}=7$, a inte- 
(a)

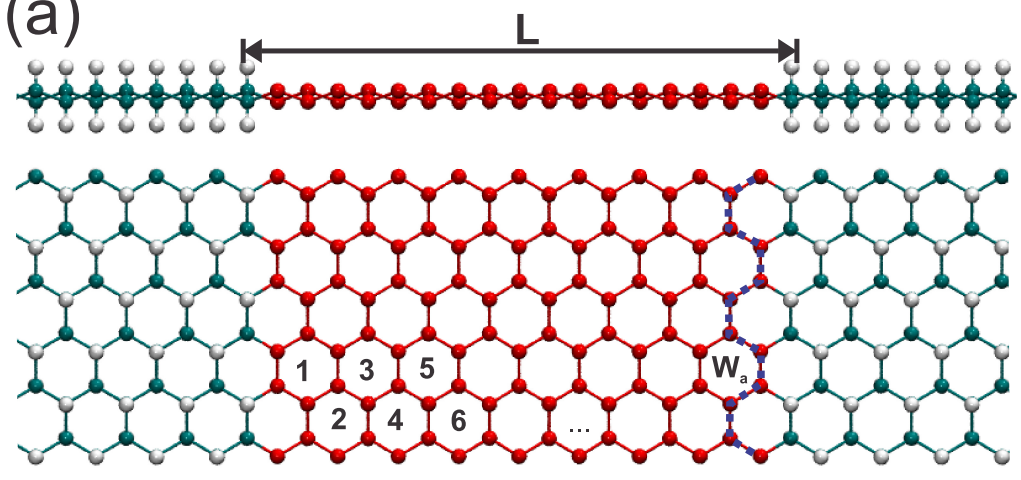

(b)

$\mathbf{L}$
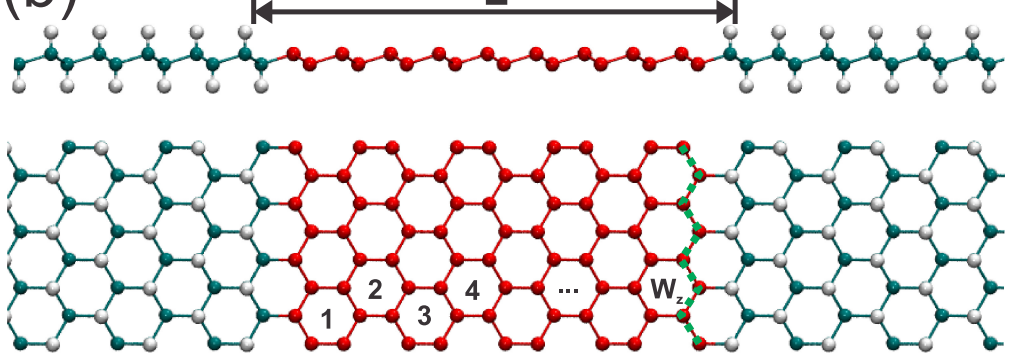

Figura 5.5: Visões laterais e superiores das representações esquemáticas das nanoestradas de germaneno com interfaces: (a) armchair, (b) zigzag. Os átomos de Ge do germaneno são representados em vermelho, enquanto que os átomos de Ge do germanano são representados em verde e os átomos de $\mathrm{H}$ são representados em branco. As larguras dessas nanoestradas são representadas pelo número de hexágonos $W_{a}$ ou $W_{z}$ mostrados na figura. 
ração entre interfaces torna-se muito fraca para abrir um gap de energia, e os estados topológicos de interface começam a surgir. Na Fig. 5.6(a) é mostrada a variação dos gaps de energia em função da largura das nanoestradas zigzag. Na Fig. 5.6(b) mostramos as estruturas de bandas calculadas para $W_{z}=16$ (painél à esquerda), enquanto que no painél à direita é mostrado uma magnificação dos estados de interfaces nas proximidades do ponto $\bar{\Gamma}$. Os estados das duas interfaces nesse caso foi mantido degenerado. As funções de onda dos estados de interfaces próximos ao ponto $\bar{\Gamma}$ são mostradas na Fig. 5.6(c). Na Fig. 5.6(d) mostramos as estruturas de bandas calculadas para $W_{z}=17$ (painél à esquerda) com uma pequena distorção entre interfaces causada por relaxações, enquanto que no painél à direita é mostrado uma magnificação dos estados de interfaces nas proximidades de $K_{Z}$ mostrando a polarização de psin em cada banda. Na Fig. 5.6(e) apresentamos a função de onda polarizada de spin no espaço real para cada estado mostrado na Fig. 5.6(c).

As texturas de spin foram calculadas a partir do valor esperado das matrizes de Pauli para estados de Bloch nas proximidades do ponto $K_{Z}$. As componentes $P_{x, n}$ e $P_{y, n}$ da textura de spin mostraram comportamento quase constantes, como mostrado na Fig. 5.7. Os estados de interface 1 e 4 (como rotulados na Fig. 5.6(b)) apresentam $P_{x, n} \approx-0,2$ e $P_{y, n} \approx 0,4$; enquanto que os estados 2 e 3 apresentam $P_{x, n} \approx 0,2$ e $P_{y, n} \approx-0.4$. Baseados nesses valores, nós reconstruímos a textura de spin dos estados topológicos, mostrados pictoricamente na Fig. 5.7(b). Para cada estado de interface, a polarização de spin resultante mostraram inclinações de $\theta=25^{\circ}$ com relação ao eixo polar ortogonal ao material. Nós podemos inferir que manipulando o potencial químico na escala de $\mathrm{meV}$, podemos obter correntes elétricas passando através das interfaces, ou através de uma única interface. Esse último cenário poderia ser uma das assinaturas significativas das nanoestradas de germaneno, além das componentes de spin no plano do mate- 

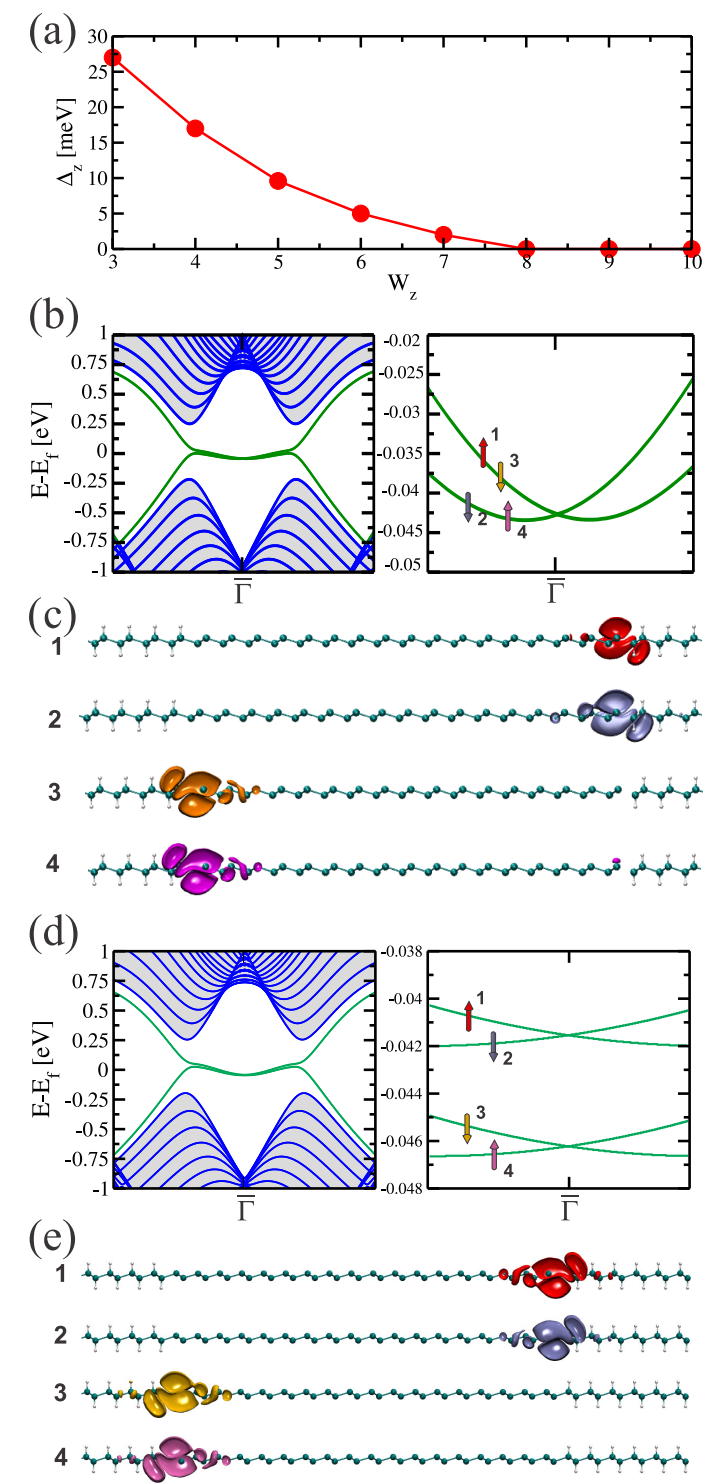

Figura 5.6: (a) Variação dos gaps de energias em função das larguras das nanoestradas zigzag. (b) Estrutura de bandas de uma nanoestrada zigzag com largura $W_{z}=16$. Uma ampliação da estrutura de bandas em torno do ponto $\Gamma$ é mostrado no painel à direita. (c) Isosuperfícies das funções de onda para os estados 1 (em vermelho), 2 (em azul), 3 (em laranja), e 4 (em rosa) da nanoestrada zigzag com largura $W_{z}=16$. As funções de onda desses estados estão majoritariamente localizadas nas regiões das interfaces zigzag. (d) Estrutura de bandas de uma nanoestrada zigzag de largura $W_{z}=17$ e pequena distorção entre interfaces causada por efeitos de relaxações. Uma ampliação da estrutura de bandas em torno do ponto $\Gamma$ é mostrado no painel à direita. (e) Isosuperfícies das funções de onda dos estados de interfaces 1 (em vermelho), 2 (em azul), 3 (em laranja), e 4 (em rosa) da nanoestrada zigzag de largura $W_{z}=17$ e distorção entre interfaces. 
rial. Essa característica poderia ser observada experimentalmente em um dispositivo de efeito Hall quântico na ausência de campos magnéticos externos.

(a)
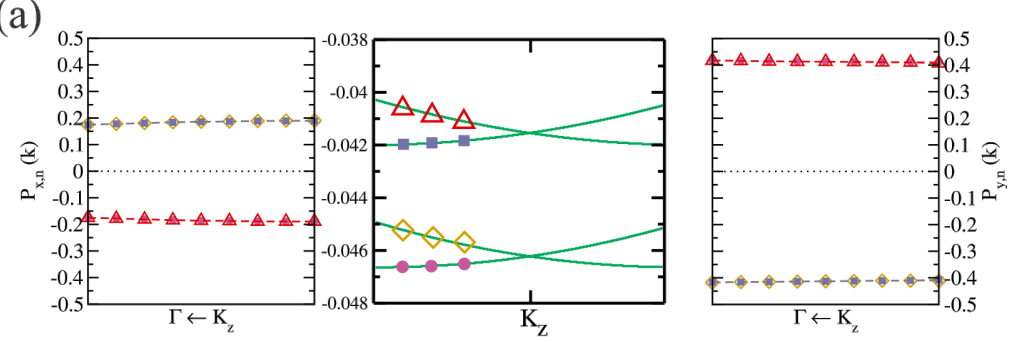

(b)

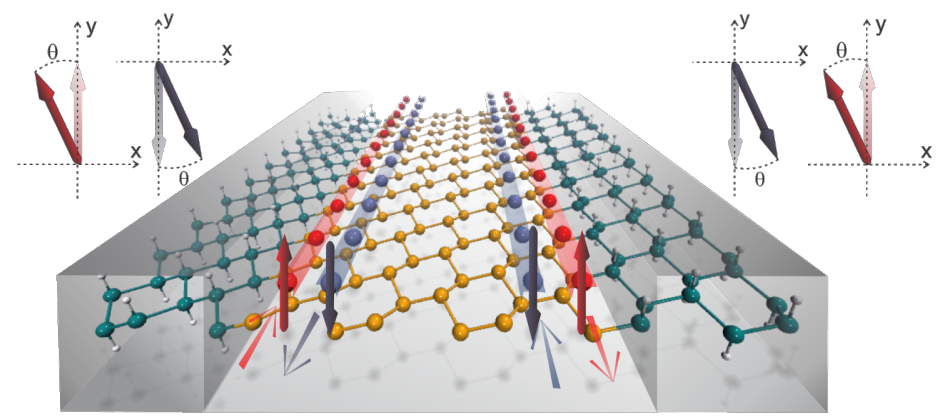

Figura 5.7: (a) Componentes das polarizações de spin $P_{x, n}$ (esquerda) e $P_{y, n}$ (direita) para os estados de interfaces rotulados simbolicamente no painel central. (b) Representação pictórica das correntes polarizadas de spin nas nanoestradas de germaneno zigzag. As polarizações de spin são inclinadas em até $\theta=25^{\circ}$ em relação ao eixo polar perpendicular ao plano do material.

As estruturas de bandas de nanoestradas de germaneno com interfaces armchair também foram estudadas em função das larguras, como mostrado na Fig.5.8. As estruturas de bandas obtidas mostraram três classes distintas de bandas. Na Fig.5.8(a) nós mostramos três sistemas representativos, $W_{a}=30,31$ e 32 . Essa variedade de estruturas de bandas mostraram flutuações de gaps de energias com quase-periodicidade $\Delta W=3$, como mostrado na Fig.5.8(b).

Além dos pequenos gaps de energias para a classe $W_{a}=3 N, N=$ $1,2, \ldots$, todos os cálculos mostraram gaps de energias não-nulo no domínio de larguras de nanoestradas estudadas. Na Fig.5.8(c) nós 

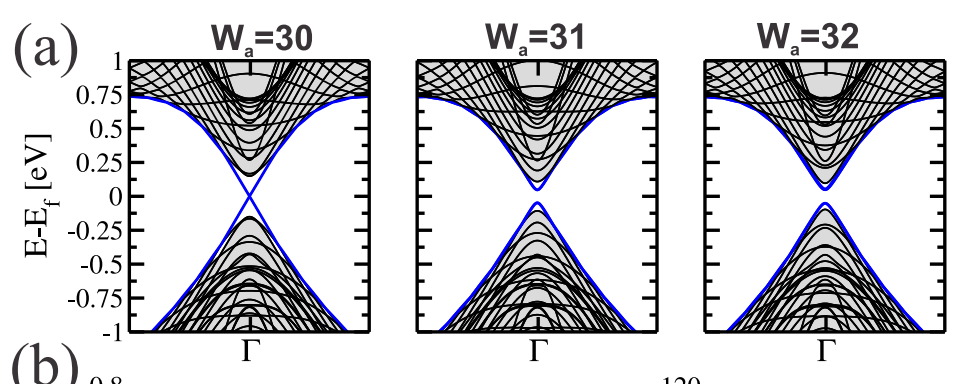

(b)
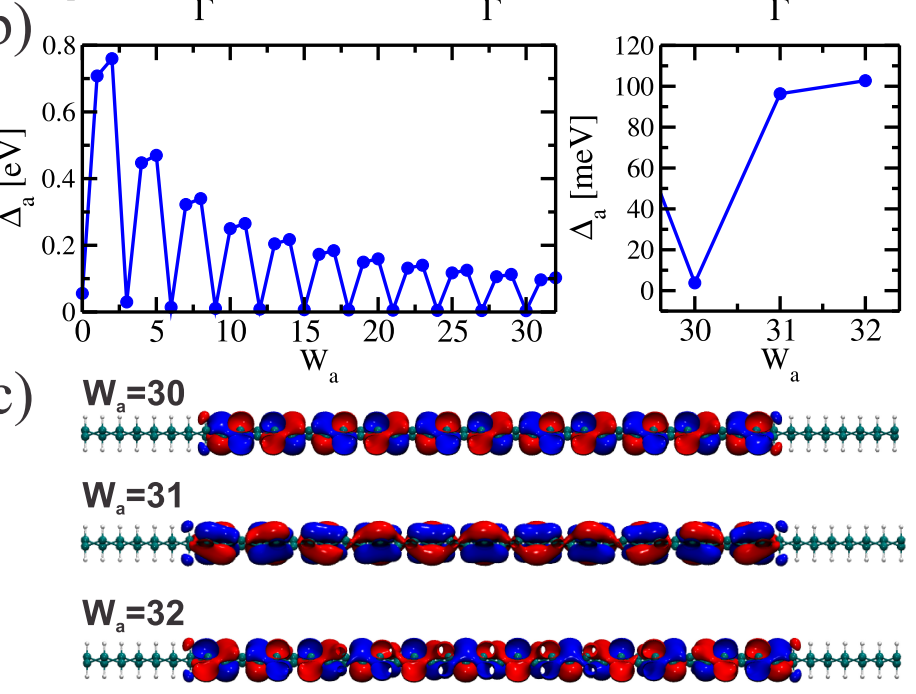

Figura 5.8: (a) Estruturas de bandas para nanoestradas armchair para larguras: $W_{a}=30, W_{a}=31$, e $W_{a}=32$. Os estados de interfaces são representados em azul, enquanto que os estados de "bulk" são representados em preto. (b) Variação dos gaps de energias em função das larguras das nanoestradas. Uma ampliação dos gaps de energias para as larguras entre 30 e 32 é mostrada no painel à direita. (c) Isosuperfícies das funções de onda (HOMO) para valores positivos (azul) e negativos (vermelho) para nanoestradas de larguras $W_{a}=30,31$, e 32 . 
mostramos que as funções de onda (para HOMO) são delocalizadas através de toda a nanoestradas. Recentes resultados obtidos por Ezawa e Nagaosa [77] para nanofitas de siliceno mostraram resultados semelhantes. Nanofitas com bordas armchair foram caracterizadas pela ausência de estados localizados nas bordas, diferente das nanofitas com bordas zigzag. Em outras palavras, o comprimento de decaimento dos orbitais de bordas para terminações zigzag e armchair são completamente diferentes.

\subsection{Conclusão}

Nós mostramos que o efeito Hall quântico de spin pode ser observado em nanoestradas de germaneno imersas em germanano em condições experimentais realistas. Essas nanoestruturas poderiam ser obtidas através de dissociação local de hidrogênio do germanano na forma de uma faixa (1D) com bordas zigzag e armchair. Além do QSHE à temperaturas acessíveis, nós encontramos componentes de polarizações de spin no plano e fora-do-plano que levaram à texturas de spin com inclinações de até $25^{\circ}$ com relação ao eixo polar normal ao material, e estados de interface nãodegenerados. 


\section{Ligas desordenadas $\mathrm{Si}_{x} \mathbf{G e}_{1-x}$ em duas dimensões}

Order is Heaven's first law; and this confess, Some are and must be greater than the rest.

\section{Alexander Pope}

\subsection{Introdução}

Materiais como o siliceno [38, 39, 68, 69] e o germaneno [40] são isolantes topológicos em duas dimensões (2D). Como mostrado no capítulo anterior (Capítulo 5), essa classe de materiais podem apresentar efeitos como o efeito Hall quântico de spin [12,37]. Além disso, materiais como o siliceno e o germaneno apresentam geometria com distorção vertical entre sub-redes que os tornam suceptíveis à campos elétricos externos aplicados perpendicularmente ao plano do material 2D. Como mostrado nas referências [78], a aplicação de um campo elétrico externo pode induzir uma redução do gap de energia do material de modo que haja uma transição da fase isolante topológica para o estado metálico ou a fase isolante 
trivial (também conhecido como isolante de bandas ou isolante ordinário). A análise do gap de energia do siliceno [ver Fig. 6.1(a)] em função do campo elétrico aplicado é mostrado na Fig. 6.1(b). A fase metálica existe para um valor do campo crítico $\left|E_{c}\right|$. Fenômeno semelhante ocorre com o germaneno, mas com o valor do campo crítico diferente do siliceno [78].

(a)

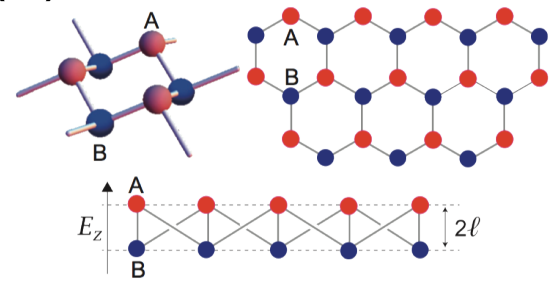

(b)

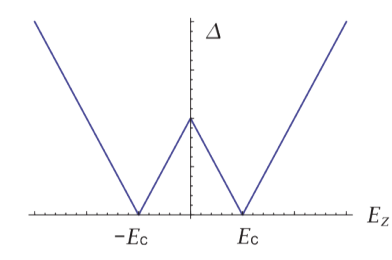

Figura 6.1: (a) Representação esquemática da estrutura do siliceno, e a direção $E_{z}$ do campo elétrico aplicado. (b) Comportamento do gap de energia do siliceno em função do campo elétrico aplicado. Para campos elétricos $\left|E_{z}\right|<E_{c}$, o siliceno apresenta topologia de bandas não-trivial, e topolgias de bandas triviais para os outros campos elétricos. Figuras extraídas da referência [78].

O comportamento do gap de energia em função do campo elétrico aplicado pode ser visto como uma das assinaturas desses isolantes topológicos com invariante $\mathbf{Z}_{2}=1$. Os caráteres das funções de onda no HOMO e LUMO também podem indicar a topologia de bandas $\mathbf{Z}_{2}$ do material.

Nesse capítulo vamos estudar a fase não-trivial dos isolantes topológicos formados por ligas desordenadas hexagonais de $\mathrm{Si}_{x} \mathrm{Ge}_{1-x}$. Embora o siliceno e o germaneno sejam isolantes topológicos 2D, uma liga do tipo $\mathrm{Si}_{x} \mathrm{Ge}_{1-x}$ introduz desordens estruturais que alteram as propriedades eletrônicas, e consequentemente, podem alterar a topologia de bandas não-trivial do material.

As propriedades estruturais, termodinâmicas, e eletrônicas das ligas $\mathrm{Si}_{x} \mathrm{Ge}_{1-x}$ foram estudadas a partir de métodos de primeiros princípios baseados na Teoria do Funcional da Densidade (ver Apêndice A) implementada no código OpenMX [75]. Foram utilizados 
funcionais de troca-correlação na aproximação GGA-PBE, e acoplamentos spin-órbitas nos pseudopotenciais de norma-conservada completamente relativísticos.

\subsection{Ligas desordenadas e SQS}

O estudo de ligas desordenadas desse capítulo foi realizado usando estruturas especiais quase-aleatórias (SQS, acrônimo do termo em inglês Special Quasirandom Structure) como descrito nas referências $[79,80]$. Essa estruturas são criadas de forma que imitem uma liga completamente desordenadas. Para alcançar esse objetivo, o método SQS gera pequenas estruturas periódicas de forma que as interações entre os primeiros vizinhos sejam descritas por funções de correlação menores que um valor crítico. Para uma liga binária com espécies atômicas Si e Ge, é definido um spin fictício de modo que $S_{\mathrm{Si}}=-1$ e $S_{\mathrm{Ge}}=+1$. A função de correlação do método SQS para uma configuração $\sigma$ é dada por

$$
\Pi_{m}(\sigma)=\frac{1}{Z_{m} N} \sum_{i, j} \Delta_{m}(i, j) S_{i} S_{j},
$$

onde $Z_{m}$ é o número de $m$-ésimos vizinhos, $N$ é o número total de átomos na supercélula SQS, e $\Delta_{m}(i, j)$ é uma função que é 1 se o sítio $i$ é $m$-ésimo vizinho do sítio $j$, e 0 caso contrário. Essa função de correlação pode ser comparada com a função de correlação da estrutura completamente aleatória $(R)$, que é

$$
\Pi_{m}(R)=(2 x-1)^{2},
$$

onde $x$ é a concentração de Si na liga.

O desvio da função de correlação SQS em relação à função de correlação da estrutura aleatória é dado por

$$
\delta \Pi_{m}(\sigma)=\left|\Pi_{m}(\sigma)-\Pi_{m}(R)\right| .
$$


Uma estrutura é chamada de estrutura especial quase-aleatória se as funções $\delta \Pi_{m}(\sigma)$ são menores que um valor crítico $\varepsilon$ para um conjunto de $m$ primeiros vizinhos.

$\mathrm{Na}$ nossa liga $\mathrm{Si}_{x} \mathrm{Ge}_{1-x}$ foram calculadas as funções de correlação de sistemas com $N=98$ átomos (supercélula $7 \times 7$ ), e concentrações de Si dadas por $x=0.0,0.184,0.265,0.5,0.736$, e 1.0. Uma estrutura gerada com o método SQS para $x=0.5$ é mostrada na Fig. 6.2(a).

(a)

(b)
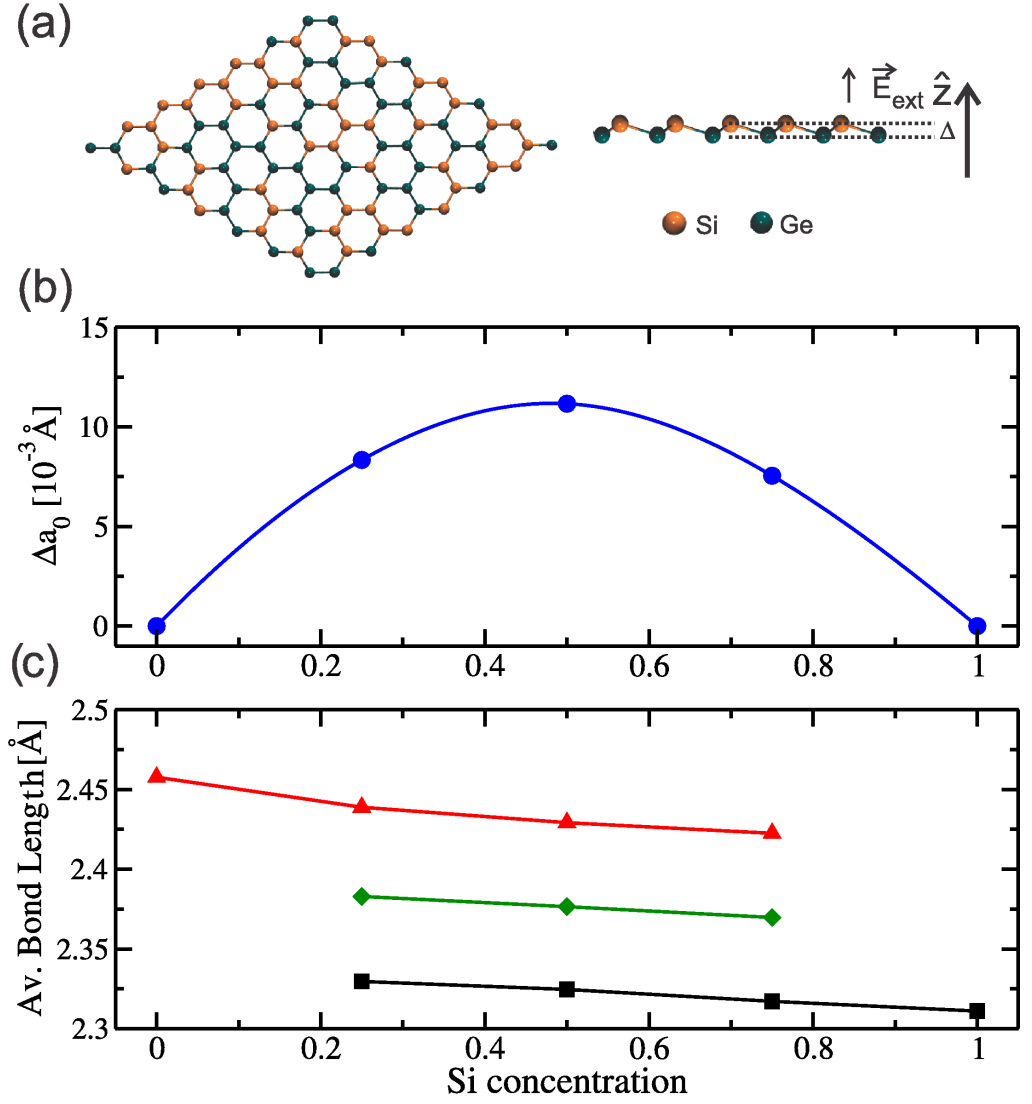

Figura 6.2: (a) Visão superior (à esquerda) e lateral (à direita) da representação esquemática da liga $\mathrm{Si}_{x} \mathrm{Ge}_{1-x}$ para $x=0.5$. (b) Desvio das constantes de rede em torno da linearidade de acordo com a equação 6.4. (c) Evolução das distâncias de ligações $d_{\mathrm{GeGe}}, d_{\mathrm{GeSi}}$, e $d_{\mathrm{SiSi}}$ em função da concentração $x$. As distâncias Si-Si são representadas em preto, as distâncias Si-Ge são representadas em verde, e as distâncias Ge-Ge são representadas em vermelho. 
As contantes de rede encontradas variaram de $a_{\mathrm{Si}}^{0}=3.87 \AA$ até $a_{\mathrm{Ge}}^{0}=4.06 \AA$. Para o sitema $0<x<1$ foram calculadas as constantes de rede $a_{\mathrm{eq}}^{0}(x)$ e os desvios da linearidade

$$
\Delta a^{0}(x)=\left[x a_{\mathrm{Si}}^{0}+(1-x) a_{\mathrm{Ge}}^{0}\right]-a_{\mathrm{eq}}^{0}(x) .
$$

Os resutaldos encontrados para $\Delta a^{0}(x)$ são mostrados na Fig. 6.2(b). As funções $\Delta a^{0}(x)$ encontradas para a liga hexagonais 2D foram semelhantes as encontradas para ligas $\mathrm{Si}_{x} \mathrm{Ge}_{1-x}$ com estrutura de blendas. As variações em torno da linearidade foram muito pequenas, no máximo $0.01 \AA ̊$ para $x=0.5$.

Os comprimentos de ligações $d_{\mathrm{GeGe}}, d_{\mathrm{GeSi}}$, e $d_{\mathrm{SiSi}}$ foram calculados para várias concentrações de Si e Ge, mostrados na Fig. 6.2(c). Como em $x=0.0$ temos apenas átomos de Ge, os comprimentos de ligações $d_{\mathrm{GeSi}}$ e $d_{\mathrm{SiSi}}$ não foram bem definidos em $x=0.0$. Situação análoga ocorre em $x=1.0$ para $d_{\mathrm{GeGe}}$ e $d_{\mathrm{GeSi}}$. Assim como os desvios da linearidade das constantes de rede, os comprimentos de ligações $d_{\mathrm{GeGe}}, d_{\mathrm{GeSi}}$, e $d_{\mathrm{SiSi}}$ também foram bem próximos da liga $\mathrm{Si}_{x} \mathrm{Ge}_{1-x}$ com estrutura de blenda [81]. Outro parâmetro geométrico das ligas hexagonais $2 \mathrm{D}$ de $\mathrm{Si}_{x} \mathrm{Ge}_{1-x}$ que foram analisados foram as alturas das distorções verticais fora dos planos. Para o siliceno temos $\Delta=0.49 \AA$, enquanto que para o germaneno temos $\Delta=0.71$ $\AA$ A. Para as ligas com concentrações $x=0.265,0.5$, e 0.735 as alturas encontradas foram $0.66 \AA$, $0.61 \AA$, e $0.56 \AA$, respectivamente.

As energias de formações foram calculadas para as ligas para várias concentrações. Essas energias são definidas por $\Delta E(x)=$ $E_{\mathrm{Si}_{\mathrm{x}} \mathrm{Ge}_{1-\mathrm{x}}}-\left[x E_{\mathrm{Si}}+(1-x) E_{\mathrm{Ge}}\right]$, onde $E_{\mathrm{Si}_{\mathrm{x}} \mathrm{Ge}_{1-\mathrm{x}}}$, $E_{\mathrm{Si}}$, e $E_{\mathrm{Ge}}$ são as energias totais por átomo para os sistemas $\mathrm{Si}_{x} \mathrm{Ge}_{1-x}$, siliceno, e germaneno. Os valores encontrados são mostraos na Tabela 6.1, e foram até quatro vezes maiores que as ligas $\mathrm{Si}_{x} \mathrm{Ge}_{1-x}$ com estrutura de blenda [81].

Um outro parâmetro importante é a temperatura crítica $T_{c}(x)$ para decomposição da liga. Uma estimativa dessa temperatura crí- 
Tabela 6.1: Energias de formações das ligas, $\Delta E(x)$, em $\mathrm{meV} /$ átomo, e as temperaturas críticas de decomposição $T_{c}(x)$, em K.

\begin{tabular}{ccc}
\hline$x$ & $\Delta E(x)$ & $T_{c}(x)$ \\
\hline 0.265 & 13.06 & 262 \\
0.50 & 17.58 & 294 \\
0.735 & 12.71 & 255 \\
\hline \hline
\end{tabular}

tica é

$$
T_{c}(x)=\frac{\Delta E(x)}{\Delta S(x)},
$$

onde a entropia de configuração é

$$
\Delta S(x)=-k_{B}\left[x \log _{10} x+(1-x) \log _{10}(1-x)\right] .
$$

As temperaturas críticas calculadas também são mostradas na Tabela 6.1 .

\subsection{Estrutura eletrônica das ligas $\mathbf{S i}_{x} \mathbf{G e}_{1-x}$ em duas dimensões}

As estruturas de bandas foram calculadas para ligas hexagonais $2 \mathrm{D}$ de $\mathrm{Si}_{x} \mathrm{Ge}_{1-x}$ para concentrações $x=0.265,0.5$, e 0.735 , como mostrado na Fig. 6.3. As estruturas de bandas de todas as configurações se mostraram semelhantes as estruturas de bandas do siliceno e do germaneno, diferindo apenas no gap de energia no ponto $K\left(\Delta_{K}\right)$. O gap de energia varia do germaneno ao siliceno pristino de $\Delta_{K}^{\mathrm{Ge}}=25.5 \mathrm{meV}$ até $\Delta_{K}^{\mathrm{Si}}=1.95 \mathrm{meV}$. Essa variação de gap de energia é mostrada na Fig. 6.4(a). Detalhes das variações dos gaps de energia em função de $x$ são mostrados na Fig. 6.4(b).

Na Fig. 6.4(a), além da variação do gap de energia $\Delta_{K}(x)$, também é mostrada a variação desse gap em torno da lineariadade. 

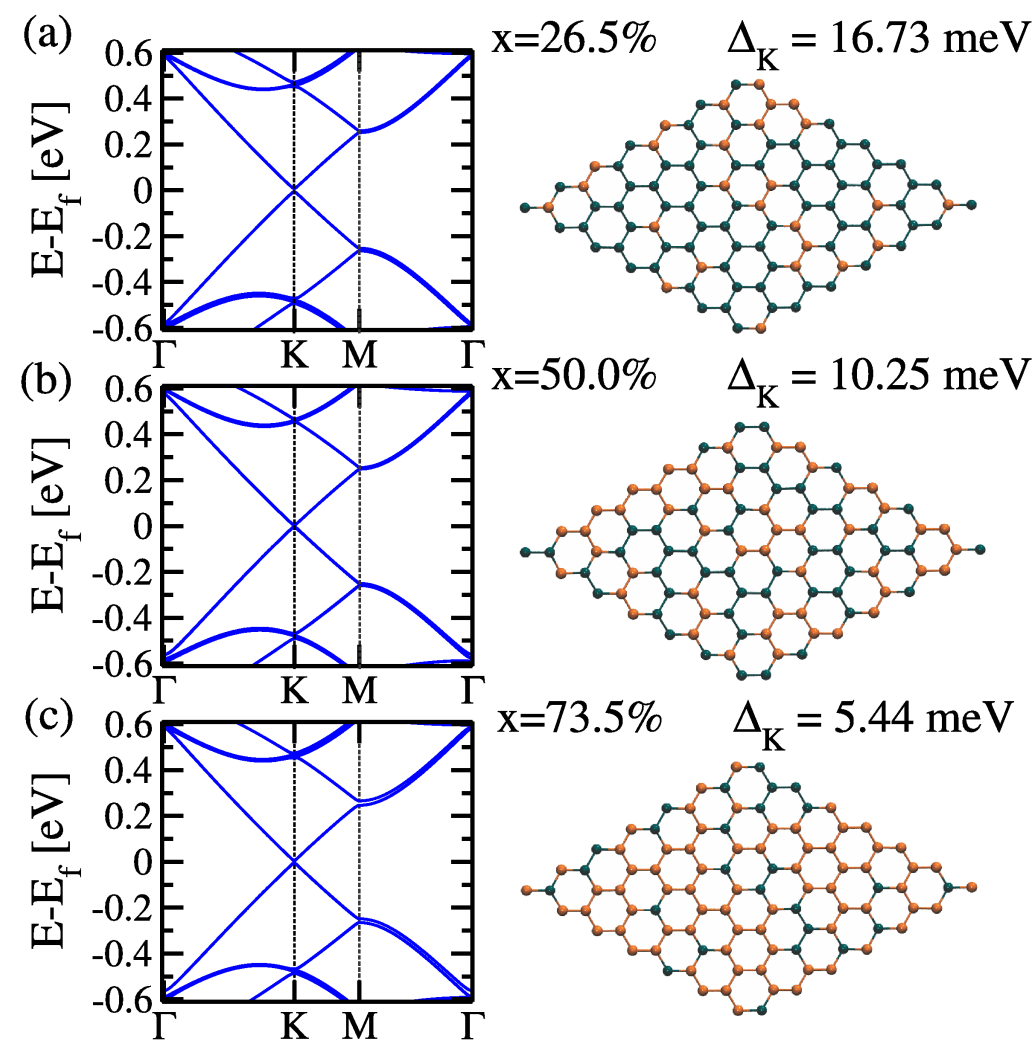

Figura 6.3: Estruturas de bandas calculadas por métodos de primeiros princípios (paineis à esquerda) e visões superiores das representações esquemáticas das geometrias das ligas $\mathrm{Si}_{x} \mathrm{Ge}_{1-x}$ para: (a) $x=0.265$, (b) $x=0.5$, e (c) $x=0.735$. Os átomos de Si são representados em laranja, enquanto que os átomos de Ge são representados em verde. 
Essa medida de variação de linearidade em $x$ do gap é dada por

$$
\Delta g(x)=\left[x \Delta_{K}^{\mathrm{Si}}+(1-x) \Delta_{K}^{\mathrm{Ge}}\right]-\Delta_{K}(x)
$$

(a)
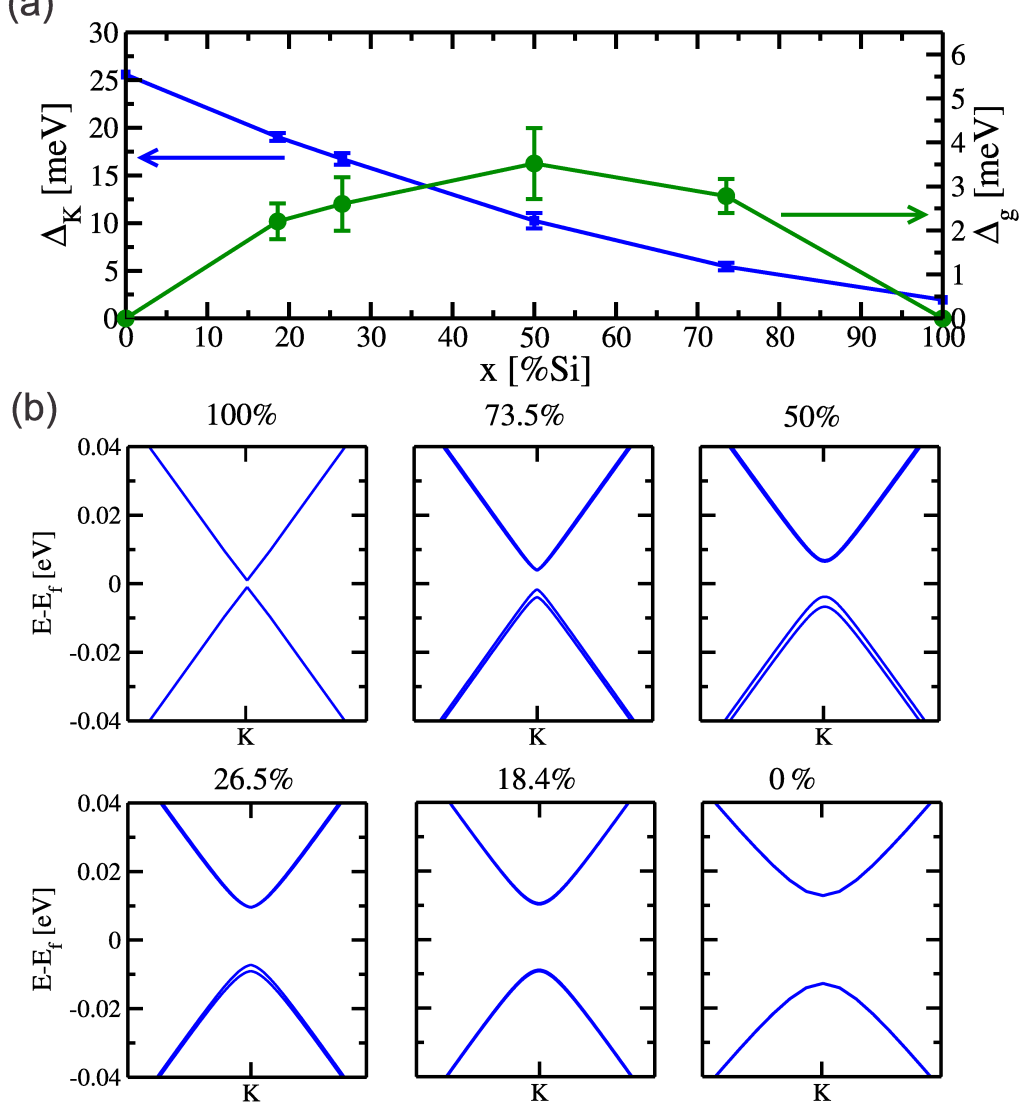

Figura 6.4: (a) Variações dos gaps de energias $\left[\Delta_{K}(x)\right]$ (em azul), e desvio da linearidade $[\Delta g(x)]$ (em verde) em função da concentração $x$ de Si. (b) Estruturas de bandas próximas ao ponto $K$ como função da concentração $x$. Essas estruturas de bandas são mostradas desde o siliceno (caso 100\%) até o germaneno (caso 0\%). O gap de energia é variados continuamente e monotonicamente, sem estados metálicos intermediários que indicariam transições de topologias de bandas.

Nós podemos notar que a variação do gap de energia do siliceno ao germaneno nunca fecha para concentrações intermediárias $(0<x<1)$. Como ambos materiais são isolantes topológicos 
com $\mathbf{Z}_{2}=1$, então por continuidade adiabática os sistemas com concentrações intermediárias também serão isolantes topológicos $\operatorname{com} \mathbf{Z}_{2}=1$. O argumento de continuidade adiabática é usado considerando que a concentração $x$ de Si pode variar continuamente. Dessa forma, podemos concluir que as ligas desordenadas hexagonais $2 \mathrm{D}$ de $\mathrm{Si}_{x} \mathrm{Ge}_{1-x}$ são isolantes topológicos para qualquer $x$. Esse resultado não é trivial. Ligas ordenadas de $\mathrm{Si}_{0.5} \mathrm{Ge}_{0.5}$ apresentam gap de energia de $7 \mathrm{meV}$, mas não é um isolante topológico. Também notamos que estruturas desordenadas que não são SQS podem apresentar estruturas de bandas metálicas, diferente dos isolantes topológicos. Não é qualquer mistura de Si e Ge em um material hexagonal 2D que apresenta a fase eletrônica dos isolantes topológicos com $\mathbf{Z}_{2}=1$.

\subsection{Efeito de campo elétrico}

Já que tanto o siliceno quanto o germaneno apresentam transições de topologias de bandas com a aplicação de um campo elétrico externo, podemos estudar esse tipo de transição para as ligas de $\mathrm{Si}_{x} \mathrm{Ge}_{1-x}$. Essa transição topológica é manifestada através de um comportamento em $W$ do gap de energia em função do campo elétrico aplicado [78], como pode ser visto na Fig. 6.5. As ligas hexagonais $2 \mathrm{D}$ de $\mathrm{Si}_{x} \mathrm{Ge}_{1-x}$ também apresentam o comportamento em $W$ do gap de energia (ver Fig. 6.5). Nesses materiais, o índice topológico é $\mathbf{Z}_{2}=1$ para intensidades de campos elétricos $E<\left|E_{c}\right|$, e $\mathbf{Z}_{2}=0$ para $E>\left|E_{c}\right|$. Os valores dos campos elétricos críticos $\left(E_{c}\right)$ variam de $E_{c}^{\mathrm{Si}}=0.2 \mathrm{~V} / \mathrm{nm}$ para o siliceno, para $E_{c}^{\mathrm{Ge}}=2.2 \mathrm{~V} / \mathrm{nm}$ para o germaneno. Para as ligas com $0<x<1$, os campos elétricos críticos encontrados foram entre $E_{c}^{\mathrm{Si}}$ e $E_{c}^{\mathrm{Ge}}$.

Para a liga com $x=0.5$ sem a aplicação do campo elétrico, o sistema apresenta um gap de energia de $\Delta_{K}=10.25 \mathrm{meV}$. Quando é aplicado um campo elétrico de $\left|E_{c}\right|=1.0 \mathrm{~V} / \mathrm{nm}$, o gap de energia 


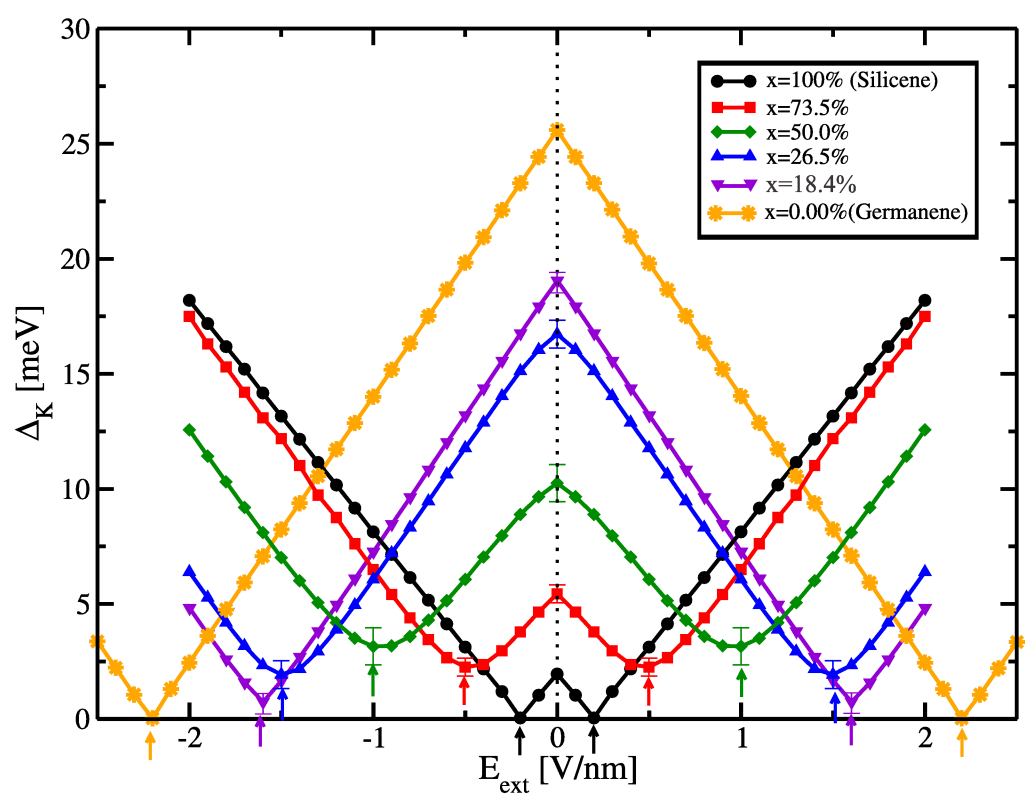

Figura 6.5: Variações dos gaps de energias em $K$ em função do campo elétrico aplicado nas ligas $\mathrm{Si}_{x} \mathrm{Ge}_{1-x}$. Os resultados são apresentados para seis concentrações $x=0$ (germaneno, em laranja), 0.184 (em roxo), 0.265 (em azul), 0.500 (em verde), 0.735 (em vermelho), e 1.00 (siliceno, em preto). Para campos elétricos $\left|E_{z}\right|<E_{c}$, as ligas apresentam topologias de bandas não-triviais (isolantes topológicos), enquanto que para campos maiores que $E_{c}$ e menores que $-E_{c}$ as topologias de bandas são triviais (isolante trivial). 
atingi um valor mínimo. Esse gap de energia ainda permanece na liga devido ao efeito de finitude da supercélula. Em supercélulas maiores o gap reminiscente com o campo elétrico crítico diminui monotonicamente, como mostrado na Fig. 6.6 para $x=0.265$. Um comportamento semelhante pode ser visto para outras concentrações de Si e Ge. Mesmo com a existência desse gap de energia para campos elétricos críticos, ainda podemos ver que temos $\mathbf{Z}_{2}=1$ para campos elétricos $E<\left|E_{c}\right|$ e $\mathbf{Z}_{2}=0$ para os outros casos.

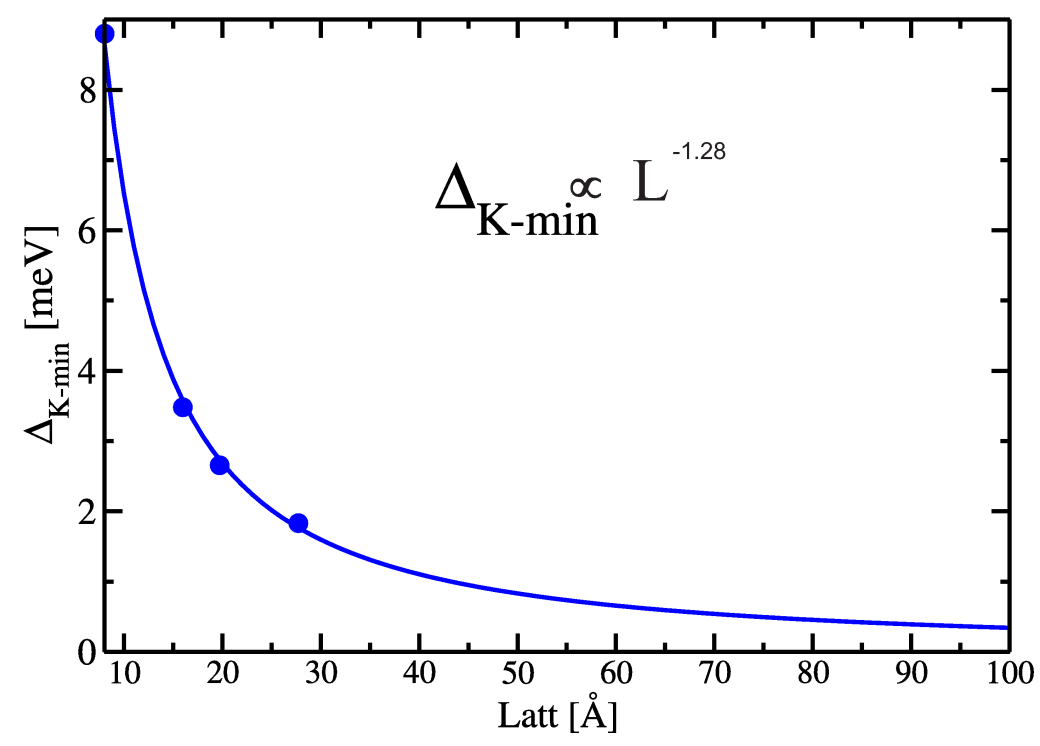

Figura 6.6: Variação do gap de energia no ponto $K$ em função da constante de rede da supercélula da liga $\mathrm{Si}_{x} \mathrm{Ge}_{1-x}$ para $x=0.265$. Efeitos de finitude da célula unitária utilzadas nos cálculos de estruturas SQS são responsáveis pelo gap de energia não-nulo nos pontos $K$ para os campos elétricos críticos.

\subsection{Conclusões}

Nós mostramos que uma liga desordenada de $\mathrm{Si}_{x} \mathrm{Ge}_{1-x}$ em duas dimensões é um isolante topológico com $\mathbf{Z}_{2}=1$, assim como o siliceno e o germaneno pristinos. É importante notar que nem toda liga de Si e Ge compartilham da mesma topologia de bandas não- 
trivial do siliceno e germaneno. Apenas em uma liga desordenada podemos ver essa fase isolante topológica. Essas ligas desordenadas também aprentam comportamento em $W$ do gap de energia em função do campo elétrico externo, e efeitos de finitude da supercélula criam um gap reminiscente que são criados por interações de longos alcances. Esses isolantes topológicos podem trazer novas oportunidades para o desenvolvimento de dispositivos, junto com a engenharia de gap obtida nas ligas desordenadas. Esses isolantes topológicos $2 \mathrm{D}$ também podem ser usados para a observação do efeito Hall quântico de spin. 


\section{Conclusões gerais}

All's well that ends well.

William Shakespeare

Nessa tese apresentamos os conceitos fundamentais da teoria dos isolantes topológicos em duas e três dimensões. A descrição desses materiais foi fundamentada em números inteiros associados à teoria de bandas que são chamados de invariantes topológicos. A partir da simetria de reversão temporal podemos encontrar que os invariantes topológicos são da classe $\mathbf{Z}_{2}$. Foi demonstrado que materiais em duas dimensões são descritos topologicamente por um invariante $\mathbf{Z}_{2}$, enquanto que materiais em três dimensões são descritos por quatro invariantes $\mathbf{Z}_{2}$.

Na parte de Resultados da tese, apresentamos o estudo das propriedades eletrônicas de quatro sistemas. Dois sistemas em duas dimensões e dois sistemas em três dimensões. Mostramos que isolantes topológicos do tipo $\mathrm{Bi}_{2} \mathrm{Te}_{3}$ com falhas de empilhamentos podem exibir estados metálicos localizados na região do defeito estrutural (falha de empilhamento). Enquanto isso, isolantes topológicos do tipo $\mathrm{Bi}_{2} \mathrm{Se}_{3}$ apresentam somente pequenas reduções no gap de energia no ponto $\bar{\Gamma}$ quando há a presença de falhas de empilhamentos. Também mostramos que a interação entre os esta- 
dos topologicamente protegidos do $\mathrm{Bi}_{2} \mathrm{Se}_{3}$ e a banda de valência do GaAs origina desdobramentos do cone de Dirac no ponto $\bar{\Gamma}$. Esses resultados foram confirmados por cálculos de primeiros princípios e extendidos por um modelo Hamiltoniano efetivo de quatro bandas.

Para isolantes topológicos em duas dimensões, mostramos que podemos formar nanoestradas de germaneno imersas em germanano a partir da dissociação local de hidrogênio. Foi demonstrado que em nanoestradas de germaneno com interfaces zigzag há a presença de estados metálicos com origem na topologia de bandas não-trivial do germaneno. Esses estados topológicos de borda não foram encontrados para o caso de interfaces armchair para as larguras de nanoestradas estudadas. Também mostramos que ligas desordenadas hexagonais de $\mathrm{Si}_{x} \mathrm{Ge}_{1-x}$ em duas dimensões são isolantes topológicos. Finalmente, mostramos que a desordem nas ligas hexagonais de $\mathrm{Si}_{x} \mathrm{Ge}_{1-x}$ é fundamental na manutenção da topologia de bandas não-trivial compartilhada pelo siliceno e germaneno.

Finalmente, concluímos que a partir de cálculos de primeiros princípios da estrutura eletrônica de materiais em duas e três dimensões, podemos demonstrar as consequências físicas da topologia de bandas não-trivial dos isolantes topológicos. Essas consequências mostraram-se de acordo com a topologia de bandas $\mathbf{Z}_{2}$, e apresentaram potenciais aplicações em spintrônica e computação quântica topológica. 


\section{Parte III}

\section{Apêndices}





\section{Teoria do Funcional da Densidade}

A Teoria do Funcional da Densidade (DFT, acrônimo do termo em inglês Density Functional Theory) é uma abordagem da mecânica quântica que formula que qualquer observável físico pode ser escrito como um funcional da densidade eletrônica

$$
n(\mathbf{r})=N \int d^{3} r_{2} \ldots d^{3} r_{N}\left|\psi\left(\mathbf{r}, \mathbf{r}_{2}, \ldots, \mathbf{r}_{N}\right)\right|^{2}
$$

onde $\psi\left(\mathbf{r}, \mathbf{r}_{2}, \ldots, \mathbf{r}_{N}\right)$ é a função de onda (antissimétrica) de $N$ elétrons. Enquanto que a densidade eletrônica é uma função de 3 variáveis, a função de onda é uma função de $3 N$ variáveis. Dessa forma, o custo computacional para diagonalização de problemas de muitos corpos com a DFT é muito mais baixo que os encontrados em métodos de função de onda como a teoria de Hartre-Fock e a interação de configuração.

A fundamentação da DFT é baseada nos teoremas de HohenbergKohn e nas equações de Kohn-Sham. A partir disso, é montado um ciclo de auto-consistência que pode convergir para o estado fundamental do sistema. 


\section{A.1 Teoremas de Hohenberg-Kohn}

Teorema 2. O potencial externo $V[n]$ é um funcional único da densidade $n(\mathbf{r})$.

Prova. A forma mais simples de demonstrar esse teorema é partir do argumento de redução ao absurdo. Para isso é necessário definir o potencial externo como

$$
v(\mathbf{r})=\frac{\delta V}{\delta n(\mathbf{r})}
$$

Se dois potenciais diferentes $v(\mathbf{r})$ e $v^{\prime}(\mathbf{r})$ fornecem a mesma densidade eletrônica, os seus estados correspondentes $|\psi\rangle$ e $\left|\psi^{\prime}\right\rangle$ devem fornecer os funcionais de energias totais

$$
E[\psi]=\langle\psi|(T+U+V)| \psi\rangle,
$$

$\mathrm{e}$

$$
E\left[\psi^{\prime}\right]=\left\langle\psi^{\prime}\left|\left(T+U+V^{\prime}\right)\right| \psi^{\prime}\right\rangle
$$

usando o princípio de Rayleigh-Ritz. Nas equações (A.3) e (A.4) $T$ é o operador de energia cinética, $U$ é o operador de energia potencial elétron-elétron, e $V$ é o operador de potencial externo, que inclui as interações elétron-núcleo.

Como $\left|\psi^{\prime}\right\rangle$ não é um estado que minimiza $E[\psi]$, e $|\psi\rangle$ não é um estado que minimiza $E^{\prime}\left[\psi^{\prime}\right]$, então temos as desigualdades

$$
\begin{gathered}
\langle\psi|(T+U+V)| \psi\rangle\left\langle\left\langle\psi^{\prime}|(T+U+V)| \psi^{\prime}\right\rangle\right. \\
\left\langle\psi^{\prime}\left|\left(T+U+V^{\prime}\right)\right| \psi^{\prime}\right\rangle\left\langle\left\langle\psi\left|\left(T+U+V^{\prime}\right)\right| \psi\right\rangle .\right.
\end{gathered}
$$

Essas condições podem ser escritas como

$$
\begin{aligned}
\langle\psi|H| \psi\rangle & <\left\langle\psi^{\prime}|H| \psi^{\prime}\right\rangle \\
& <\left\langle\psi^{\prime}\left|H^{\prime}\right| \psi^{\prime}\right\rangle+\left\langle\psi\left|\left(V-V^{\prime}\right)\right| \psi\right\rangle
\end{aligned}
$$


que fornece

$$
E<E^{\prime}+\int d^{3} r n(\mathbf{r})\left[v(\mathbf{r})-v^{\prime}(\mathbf{r})\right] .
$$

Analogamente a inequação (A.8) podemos obter

$$
E^{\prime}<E+\int d^{3} r n(\mathbf{r})\left[v(\mathbf{r})-v^{\prime}(\mathbf{r})\right] .
$$

Juntas, essas equações nos fornece

$$
E+E^{\prime}<E+E^{\prime},
$$

que é um absurdo. Logo, se conclui que não existem dois potenciais externos $v(\mathbf{r})$ e $v^{\prime}(\mathbf{r})$ que forneçam a mesma densidade eletrônica $n(\mathbf{r})$.

Teorema 3. A densidade eletrônica exata de um sistema de muitos elétrons é a que minimiza ofuncional de energia total $E[n]$.

Prova. O funcional da energia total pode ser escrito como

$$
E[n]=F[n]+\langle\psi|V| \psi\rangle,
$$

onde $F[n]=\langle\psi|(T+U)| \psi\rangle$ é um funcional universal. Esse funcional independe do sistema. Em especial, para o estado fundamental temos

$$
E\left[n_{0}\right]=F\left[n_{0}\right]+\left\langle\psi_{0}|V| \psi_{0}\right\rangle .
$$

Do princípio variacional de Rayleigh-Ritz é conhecido que

$$
E\left[\psi_{0}\right]<E[\psi],
$$

que pode ser escrito como

$$
\left\langle\psi_{0}|(T+U)| \psi_{0}\right\rangle+\left\langle\psi_{0}|V| \psi_{0}\right\rangle\langle\langle\psi|(T+U)| \psi\rangle+\langle\psi|V| \psi\rangle .
$$


Usando a equação (A.11), finalmente obtemos

$$
E\left[n_{0}\right]<E[n],
$$

e a densidade do estado fundamental $n_{0}(\mathbf{r})$ é a que minimiza o funcional $E[n]$.

\section{A.2 Aproximação de Thomas-Fermi}

Embora a Teoria do Funcional da Densidade tenha sido desenvolvida na década de 60 [42,43], a densidade eletrônica já era utilizada para cálculos de energias totais de átomos e moléculas através do modelo de Thomas-Fermi [82,83]. Hoje em dia esse modelo pode ser visto como uma aproximação da DFT [84], chamada nessa tese de aproximação de Thomas-Fermi. Nessa aproximação não é considerada a energia de troca-correlação, a interação coulombiana elétron-elétron é dada pelo potencial de Hartree

$$
U_{H}[n]=\frac{e^{2}}{2} \int d^{3} r d^{3} r^{\prime} \frac{n(\mathbf{r}) n\left(\mathbf{r}^{\prime}\right)}{\left|\mathbf{r}-\mathbf{r}^{\prime}\right|},
$$

e o funcional da energia cinética é dado pela aproximação da densidade local [84], i.e.,

$$
T_{s}^{L D A}[n]=\int d^{3} r n(\mathbf{r}) t^{L D A}(n(\mathbf{r})),
$$

onde $t^{L D A}$ é a densidade de energia cinética para um líquido de elétrons homogêneo (por volume) que é dado por

$$
t^{L D A}(n)=\frac{3 \hbar^{2}}{10 m}(3 \pi)^{\frac{2}{3}} n^{\frac{5}{3}}
$$

para $n$ constante. Para a aproximação da densidade local (LDA, acrônimo do termo em inglês Local Density Approximation) é considerado que para cada elemento de volume $d^{3} r$ o líquido de elétrons 
é constante. Com essa aproximação, o funcional de energia cinética é dado por

$$
T[n] \approx T_{s}^{L D A}=\frac{3 \hbar^{2}}{10 m}(3 \pi)^{\frac{2}{3}} \int d^{3} r(n(\mathbf{r}))^{\frac{5}{3}}
$$

O funcional de energia total na aproximação de Thomas-Fermi torna-se então

$$
E_{T F}[n]=T_{s}^{L D A}[n]+U_{H}[n]+V[n]
$$

com

$$
V[n]=\int d^{3} r n(\mathbf{r}) v(\mathbf{r}),
$$

que deve ser minimizado satisfazendo a equação de vínculo

$$
\int d^{3} r n(\mathbf{r})=N
$$

A derivada funcional da energia total (A.20) fornece a equação de Thomas-Fermi

$$
\frac{\hbar^{2}}{2 m}\left(3 \pi^{2}\right)^{\frac{2}{3}} n^{\frac{2}{3}}+e^{2} \int d^{3} r^{\prime} \frac{n\left(\mathbf{r}^{\prime}\right)}{\left|\mathbf{r}-\mathbf{r}^{\prime}\right|}+v(\mathbf{r})+\mu=0 .
$$

Usando a equação de Thomas-Fermi (A.23) podemos calcular energias totais, e consequentemente, energias de ligações e de formações de moléculas simples. Entretanto, devido aos resultados não satisfatório obtidos pela aproximação de Thomas-Fermi, vamos considerar mais termos para a energia total no intuito de melhorar as energias de ligações que poderiam ser calculadas para moléculas e sólidos.

\section{A.2.1 Modificações da aproximação de Thomas-Fermi}

Na aproximação de Thomas-Fermi não é considerado o princípio da exclusão de Pauli. Portanto, não é considerado a energia de troca para o líquido de elétrons. Podemos considerar o termo de 
troca na aproximação de densidade local $[46,47,85]$ dado por

$$
E_{x}^{L D A}[n]=\int d^{3} r n(\mathbf{r}) \epsilon_{x}(n(\mathbf{r})),
$$

onde a densidade de energia de troca é

$$
\epsilon_{x}(n)=-\frac{3}{4} e^{2}\left(\frac{3}{\pi}\right)^{\frac{1}{3}} n^{\frac{4}{3}},
$$

como calculado na referência [85]. Com essa inclusão do termo de troca, o funcional de energia total torna-se

$$
E_{T F D}[n]=T_{s}^{L D A}[n]+U_{H}[n]+V[n]+E_{x}^{L D A}[n]
$$

e a aproximação é chamada de aproximação de Thomas-FermiDirac.

Outra modificação que pode melhorar a aproximação de ThomasFermi é feita a partir da inclusão de um termo que inclua variações de densidades para o funcional de energia cinética. Esse termo, conhecido como termo de von Weizsäcker [86] é dado por

$$
T_{W}[n]=\frac{\hbar^{2}}{16 m} \lambda \int d^{3} r \frac{|\nabla n(\mathbf{r})|^{2}}{n(\mathbf{r})},
$$

onde $\lambda$ é um parâmetro que assume o valor $\lambda=1$ para flutuações rápidas de baixas amplitudes, e $\lambda=\frac{1}{9}$ para variações lentas da densidade [87].

Apesar dessas modificações no funcional da energia total, com inclusão de termos de troca de Dirac e termo de von Weizsäcker, a aproximação de Thomas-Fermi modificada não fornece resultados satisfatórios para sistemas simples como átomos e moléculas. A energia de ligação calculada para algumas moléculas é negativa, enquanto que o resultado experimental é positivo [88]. Para cálculos de energias totais mais precisos é necessário calcular exatamente o funcional de energia cinética a partir de orbitais atômicos. 


\section{A.3 Equações de Kohn-Sham}

O funcional de energia total de um sistema pode ser escrito como

$$
E[n]=T_{s}[n]+U_{H}[n]+V[n]+E_{x c}[n],
$$

onde $T_{s}[n]$ é o funcional da energia cinética para o sistema de uma única partícula, $U_{H}[n]$ é o funcional do potencial de interação elétronelétron de Hartree, $V[n]$ é o funcional do potencial externo, e $E_{x c}[n]$ é o funcional de energia de troca-correlação. Este último termo inclui efeitos de correlação de energia cinética que não estão incluídos em $T_{s}[n]$ e efeitos de interação elétron-elétron que vão além do potencial de Hartree. A variação funcional da equação (A.28) fornece

$$
\frac{\delta E}{\delta n(\mathbf{r})}=\frac{\delta T}{\delta n(\mathbf{r})}+v_{H}(\mathbf{r})+v(\mathbf{r})+\frac{\delta E_{x c}}{\delta n(\mathbf{r})}=\mu,
$$

onde o potencial químico $\mu$ é o multiplicador de Lagrange para a minimização do funcional (A.28) sob o vínculo (A.22).

Para resolver a equação (A.29) vamos considerar um sistema de uma única partícula interagindo com um campo médio

$$
\frac{\delta T_{s}}{\delta n(\mathbf{r})}+v_{s}(\mathbf{r})=\mu
$$

Comparando as equações (A.29) e (A.30) é possível mapear o sistema de muitos elétrons com o sistema de uma única partícula em um campo médio a partir do potencial efetivo

$$
v_{s}(\mathbf{r})=v_{H}(\mathbf{r})+v(\mathbf{r})+\frac{\delta E_{x c}}{\delta n(\mathbf{r})} .
$$

A estrutura eletrônica e energia total do sistema de uma única partícula em um campo médio é obtido pela equação

$$
\left(-\frac{\hbar^{2}}{2 m} \nabla^{2}+v_{s}(\mathbf{r})\right) \phi_{i}(\mathbf{r})=\epsilon_{i} \phi_{i}(\mathbf{r})
$$


que é conhecido como equação de Kohn-Sham [43]. Os orbitais $\phi_{i}$ são chamados de orbitais de Kohn-Sham, e densidade eletrônica é dada por

$$
n(\mathbf{r})=\sum_{i}\left|\phi_{i}(\mathbf{r})\right|^{2},
$$

onde a soma é realizada sobre todos os estados ocupados.

O funcional da energia cinética depende explicitamente dos orbitais de Kohn-Sham que depende implicitamente da densidade eletrônica, obtendo dessa forma

$$
T_{s}\left[\phi_{i}(n)\right]=-\frac{\hbar^{2}}{2 m} \sum_{i} \int d^{3} r \phi_{i}^{*}(\mathbf{r}) \nabla^{2} \phi_{i}(\mathbf{r}) .
$$

\section{A.4 Funcionais de troca e correlação}

O funcional de troca-correlação $E_{x c}[n]$ não é conhecido exatamente, apenas aproximações podem ser usadas para o cálculo auto-consistente da densidade eletrônica da teoria do funcionalda de densidade. A aproximação mais simples para esse termo é a aproximação da densidade local (LDA), já apresentada para o funcional da energia cinética na aproximação de Thomas-Fermi.

\section{A.4.1 Aproximação da densidade local (LDA)}

Considerando que em cada elemento de volume $d^{3} r$ a densidade eletrônica é constante, o funcional de energia de troca-correlação pode ser escrito como

$$
E_{x c}^{L D A}[n]=\int d^{3} r n(\mathbf{r}) \epsilon_{x c}^{L D A}(n(\mathbf{r})),
$$

onde $\epsilon_{x c}^{L D A}(n)$ é a densidade de energia (por partícula) de trocacorrelação do líquido de elétrons homogêneo $[46,47]$ com densidade $n$.

Para parametrizar a densidade de energia $\epsilon_{x c}$ é útil definir o raio 


\section{de Wigner-Seitz}

$$
r_{s}=\frac{1}{a_{0}}\left(\frac{4 \pi}{3} n\right)^{-\frac{1}{3}}
$$

como o raio da esfera que contém um único elétron em unidades do raio de Bohr $\left(a_{0}\right)$. A densidade de energia de troca-correlação é dividida em

$$
\epsilon_{x c}^{L D A}=\epsilon_{x}^{L D A}+\epsilon_{c}^{L D A}
$$

onde a energia de troca é

$$
\epsilon_{x}^{L D A}=-\frac{0,4582}{r_{s}}
$$

e a energia de correlação é

$$
\begin{aligned}
\epsilon_{c}^{L D A}= & -\frac{0,1423}{1+1,9529 \sqrt{r_{s}}+0,3334 r_{s}}, \quad r_{s} \geq 1, \\
\epsilon_{c}^{L D A}= & -0,0480+0,0311 \ln r_{s} \\
& -0,0116 r_{s}+0,0020 r_{s} \ln r_{s}, \quad r_{s}<1,
\end{aligned}
$$

como pode ser visto na referência [46, 47].

A aproximação LDA fornece resultados satisfatórios para sólidos e estrutura onde a interação de van der Waals é importante, mas não fornece bons resultados para sistemas como átomos e moléculas, onde a variação da densidade eletrônica é relevante. Para estes sistemas é necessário uma aproximação melhor para o funcional de energia de troca-correlação.

\section{A.4.2 Aproximação do gradiente generalizado (GGA)}

Para sistemas onde o gradiente da densidade eletrônica é importante, uma aproximação adequada para o funcional de troca-correlação é a aproximação do gradiente generalizado (GGA, 
acrônimo do termo em inglês Generalized Gradient Approximation). Nesta aproximação a densidade de energia de troca-correlação é uma função da densidade e do gradiente da densidade

$$
E_{x c}^{G G A}=\int d^{3} r \epsilon_{x c}^{G G A}(n, \nabla n) .
$$

Uma função do gradiente generalizado bastante utilizada na literatura científica é a aproximação do gradiente generalizado de Perdew-Burke-Ernzerhof [76], dada pela função

$$
\epsilon_{x c}^{G G A}=n(\mathbf{r}) F_{P B E}(\boldsymbol{\nabla} n),
$$

onde

$$
F_{P B E}(s)=1,804-\frac{0,646}{0,804+0,220 s^{2}},
$$

e $s=\frac{|\nabla n|}{\sqrt[3]{3 \pi^{2} n^{4}}}$ é o gradiende adimensional da densidade.

Há algumas outras aproximações do gradiente generalizado na literatura científica como Meta-GGA [84] e Hiper-GGA, mas apenas a GGA/PBE e a LDA foram usadas para o estudo dos sistemas presente nesta tese. 


\section{Equação de Dirac e acoplamento spin-órbita}

Embora a equação de Schrödinger forneça bons resultados para cálculos de estrutura eletrônica e energias totais de sistemas pequenos de poucos átomos ou moléculas, há alguns problemas conceituais e quantitativos para alguns resultados.

Do ponto de vista conceitual, a equação de Schrödinger não é covariante por transformações de Lorentz. Como há derivadas temporais em primeira ordem e derivadas espaciais em segunda ordem, qualquer transformação de Lorentz para um referencial em movimento relativo ao referencial inicial não fornecerá uma equação do tipo Schrödinger. Outro problema conceitual é na origem do spin na mecânica quântica não-relativista. O conceito de spin é introduzido de forma ad hoc a partir do experimento de SternGerlach. É necessária a existência do momento magnético intrínseco (não relacionado à movimentos de rotações) para haver um acoplamento com o campo magnético que explique as deflexões observadas por Stern e Gerlach.

Do ponto de vista quantitativo, cálculos de energias totais e de estruturas eletrônicas de sistemas com átomos "pesados" não correspondem com os resultados experimentais. A diferença entre as energias totais calculadas e as medidas são atribuídas a correções 
relativistas da equação de Schrödinger.

Nesse sentido, vamos deduzir equações de onda relativistas que recuperem a equação de Schrödinger no limite de baixas energias (ou baixas velocidades) e forneçam resultados quatitativos satisfatórios.

\section{B.1 Equação de Dirac}

Uma dedução para a equação de onda para a mecânica quântica relativista foi proposta por Dirac em 1928 [89]. Nessa proposta, a equação de onda é escrita em um ansatz de uma equação diferencial de primeira ordem no tempo e no espaço. O ansatz da equação de Dirac é

$$
H|\Psi\rangle=\left(\boldsymbol{\alpha} \cdot \mathbf{p} c+\beta m c^{2}\right)|\Psi\rangle,
$$

onde os coeficientes $\boldsymbol{\alpha}=\left(\alpha_{1}, \alpha_{2}, \alpha_{3}\right)$ e $\beta$ são determinados de forma que satisfaçam

$$
H^{2}|\Psi\rangle=\left(\mathbf{p}^{2} c^{2}+m^{2} c^{4}\right)|\Psi\rangle .
$$

A partir das equações (B.1) e (B.2) podemos determinar a natureza dos coeficientes $\alpha$ e $\beta$. Substituindo a equação (B.1) na equação (B.2) obtemos

$$
\begin{aligned}
H^{2} & =\left(\alpha_{i} p_{i} c+\beta m c^{2}\right)\left(\alpha_{j} p_{j} c+\beta m c^{2}\right) \\
& =\left[\alpha_{i}^{2} p_{i}^{2} c^{2}+\left(\alpha_{i} \alpha_{j}+\alpha_{j} \alpha_{i}\right) p_{i} p_{j} c^{2}+\left(\alpha_{i} \beta+\beta \alpha_{i}\right) p_{i} m c^{3}+\beta^{2} m^{2} c^{4}\right] .
\end{aligned}
$$

Comparando agora essa última equação com a equação (B.2), obtemos

$$
\alpha_{1}^{2}=\alpha_{2}^{2}=\alpha_{3}^{2}=\beta^{2}=1,
$$

e as relações de anticomutação

$$
\left\{\alpha_{i}, \beta\right\}=0,\left\{\alpha_{i}, \alpha_{j}\right\}=0, \text { para } i \neq j .
$$

Como os coeficientes $\alpha_{i}$ e $\beta$ anticomutam, eles não podem assu- 
mir valores escalares.

Vamos considerar que os coeficientes $\alpha_{i}$ e $\beta$ são matrizes quadradas atuando em estados $|\psi\rangle$ que são matrizes coluna.

A menor dimensionalidade para as matrizes $\alpha_{i}$ e $\beta$ são matrizes $4 \times 4$. Os estados $|\psi\rangle$ serão matrizes coluna na forma

$$
|\Psi\rangle=\left(\begin{array}{c}
\left|\chi_{1}\right\rangle \\
\left|\chi_{2}\right\rangle \\
\left|\chi_{3}\right\rangle \\
\left|\chi_{4}\right\rangle
\end{array}\right)
$$

Qualquer conjunto de matrizes $4 \times 4$ que satisfaçam as condições (B.3) e (B.4) podem ser escolhidas como suas representações. A escolha mais comum para essas matrizes é a representação de Dirac-Pauli:

$$
\boldsymbol{\alpha}=\left(\begin{array}{ll}
0 & \boldsymbol{\sigma} \\
\boldsymbol{\sigma} & 0
\end{array}\right), \quad \beta=\left(\begin{array}{cc}
\mathbb{1}_{2 \times 2} & 0 \\
0 & -\mathbb{1}_{2 \times 2}
\end{array}\right)
$$

Como a representação usada é escrita em blocos $2 \times 2$, podemos escrever o estado quântico como

$$
|\Psi\rangle=\left(\begin{array}{c}
|\phi\rangle \\
|\psi\rangle
\end{array}\right)
$$

onde

$$
|\phi\rangle=\left(\begin{array}{c}
\left|\chi_{1}\right\rangle \\
\left|\chi_{2}\right\rangle
\end{array}\right), \quad|\psi\rangle=\left(\begin{array}{c}
\left|\chi_{3}\right\rangle \\
\left|\chi_{4}\right\rangle
\end{array}\right) .
$$

Nessa representação das matrizes $\alpha$ e $\beta$ a equação de Dirac se torna o conjunto de equações acopladas

$$
\begin{gathered}
\left(H-m c^{2}\right)|\phi\rangle=(\boldsymbol{\sigma} \cdot \mathbf{p})|\psi\rangle \\
\left(H+m c^{2}\right)|\psi\rangle=(\boldsymbol{\sigma} \cdot \mathbf{p})|\phi\rangle .
\end{gathered}
$$

A interação da partícula com um potencial externo $V$ é dada 
pela substituição mínina

$$
H \longrightarrow H-V,
$$

tornando as equações de Dirac

$$
\begin{aligned}
& \left(H-V-m c^{2}\right)|\phi\rangle=(\boldsymbol{\sigma} \cdot \mathbf{p})|\psi\rangle, \\
& \left(H-V+m c^{2}\right)|\psi\rangle=(\boldsymbol{\sigma} \cdot \mathbf{p})|\phi\rangle .
\end{aligned}
$$

\section{B.1.1 Limite de baixas energias}

Quando o sistema possui baixas energias, devemos obter do tipo Schrödinger. Considerando que os autovalores do Hamiltoniano serão escritos como $E+m c^{2}$, onde $E$ é a energia que sobressai a energia de repouso $m c^{2}$, e desacoplando as equações de Dirac para $|\psi\rangle$ temos

$$
E|\psi\rangle=\frac{1}{2 m}(\boldsymbol{\sigma} \cdot \mathbf{p})\left[1+\frac{E-V}{2 m c^{2}}\right]^{-1}(\boldsymbol{\sigma} \cdot \mathbf{p})|\psi\rangle+V|\psi\rangle
$$

Usando a expansão aproximada

$$
\left(1+\frac{E-V}{2 m c^{2}}\right)^{-1} \approx 1-\frac{E-V}{2 m c^{2}}
$$

e a relação

$$
\mathbf{p} V-V \mathbf{p}=-i \hbar \nabla V
$$

finalmente encontramos

$$
E|\psi\rangle=\frac{\mathbf{p}^{2}}{2 m}|\psi\rangle-\frac{\mathbf{p}^{4}}{8 m^{3} c^{2}}|\psi\rangle+V(\mathbf{r})|\psi\rangle-\frac{\hbar^{2}}{4 m^{2} c^{2}} \boldsymbol{\nabla} V \cdot \boldsymbol{\nabla}|\psi\rangle+\frac{\hbar}{4 m^{2} c^{2}} \boldsymbol{\sigma} \cdot(\boldsymbol{\nabla} V \times \mathbf{p})|\psi\rangle .
$$

Dessa equação podemos observar que há três termos de correções relativistas para a equação de Schrödinger. 
O primeiro termo é a correção de energia cinética

$$
T_{\text {rel }}=-\frac{\mathbf{p}^{4}}{8 m^{3} c^{2}} .
$$

O segundo termo é o termo de Darwin

$$
H_{D}=-\frac{\hbar^{2}}{4 m^{2} c^{2}} \nabla V \cdot \nabla .
$$

E o terceiro termo é o acoplamento spin-órbita

$$
H_{S O}=\frac{\hbar}{4 m^{2} c^{2}} \boldsymbol{\sigma} \cdot(\boldsymbol{\nabla} V \times \mathbf{p}) .
$$

\section{B.2 Acoplamento spin-órbita}

Considerando que o potencial $V(\mathbf{r})$ é um potencial central, temos que

$$
\nabla V(r)=\frac{1}{r} \frac{d V(r)}{d r} \mathbf{r} .
$$

Substituindo a equação (B.21) no termo $\sigma \cdot(\nabla V \times \mathbf{p})$ do acoplamento spin-órbita encontramos

$$
\frac{1}{r} \frac{d V}{d r}(\mathbf{r} \times \mathbf{p}) \cdot \boldsymbol{\sigma}=\frac{1}{r} \frac{d V}{d r} \mathbf{L} \cdot \boldsymbol{\sigma} .
$$

Lembrando que o operador de spin é dado por $\mathbf{S}=\frac{\hbar}{2} \sigma$, chegamos na famosa expressão

$$
H_{S O}=\frac{1}{2 m^{2} c^{2}} \frac{1}{r} \frac{d V}{d r} \mathbf{L} \cdot \mathbf{S} .
$$

Na base $s_{z}$, o acoplamento spin-órbita torna-se

$$
H_{S O}=\frac{1}{2 m^{2} c^{2}} \frac{1}{r} \frac{d V}{d r}\left[L_{z} S_{z}+\frac{1}{2}\left(L_{+} S_{-}+L_{-} S_{+}\right)\right] .
$$


Escrevendo as funções de onda como

$$
\left|\psi_{n \mathbf{k}}\right\rangle=\left(\begin{array}{c}
\left|\psi_{n \mathbf{k}}^{\uparrow}\right\rangle \\
\left|\psi_{n \mathbf{k}}^{\downarrow}\right\rangle
\end{array}\right)
$$

o operador Hamiltoniano pode ser escrito como

$$
H_{S O}=\frac{\hbar}{4 m^{2} c^{2}} \frac{1}{r} \frac{d V}{d r}\left(\begin{array}{cc}
L_{z} & 2 L_{-} \\
2 L_{+} & -L_{z}
\end{array}\right) .
$$

Essa interação pode ser incluída na parte não-local do pseudopotencial ou da interação "all-electron" do PAW. 


\section{Invariantes $\mathrm{Z}_{2}$ em ondas planas}

Os estados de Bloch podem ser expandidos na base de ondas planas da seguinte forma

$$
u_{n \mathbf{k}}^{\sigma}(\mathbf{r})=\frac{1}{\sqrt{\Omega}} \sum_{\mathbf{G}} c_{n}^{\sigma}(\mathbf{G}) \mathrm{e}^{i \mathbf{G} \cdot \mathbf{r}}
$$

onde $n$ é o índice de banda, $\sigma$ é o índice de spin, $\Omega$ é o volume do cristal, e $\mathrm{G}$ são os vetores da rede recíproca.

Os coeficientes de ondas planas $\left[c_{n}^{\sigma}(\mathbf{G})\right]$ serão considerados normalizados nas expressões dos invariantes topológicos $\mathbf{Z}_{2}$, i.e.,

$$
\int d^{3} r u_{n \mathbf{k}}^{\sigma *}(\mathbf{r}) u_{m \mathbf{k}}^{\sigma^{\prime}}(\mathbf{r})=\sum_{\mathbf{G}} c_{n}^{\sigma *}(\mathbf{G}) c_{m}^{\sigma^{\prime}}(\mathbf{G})=\delta_{n m} \delta_{\sigma \sigma^{\prime}}
$$

\section{C.1 Invariante $Z_{2}$ centrossimétrico}

Para calcular os invariantes topológicos $\mathbf{Z}_{2}$ em materiais centrossimétricos, é necessário apenas calcular as paridades dos estados de Bloch nos pontos TRIM da zona de Brillouin, como mostrado na equação (2.78).

A paridade de um estado de Bloch $\left|u_{n \mathbf{k}}^{\sigma}\right\rangle$ pode ser calculada atra- 
vés do valor esperado do operador $\Pi$, dado por

$$
\begin{aligned}
\xi_{2 n}\left(\Gamma_{\alpha}\right)=\left\langle u_{n \mathbf{k}}|\Pi| u_{n \mathbf{k}}\right\rangle & =\int d^{3} r u_{n \mathbf{k}}^{*}(\mathbf{r}) u_{n \mathbf{k}}(-\mathbf{r}) \\
& =\sum_{\mathbf{G}} c_{n}^{*}(\mathbf{G}) c_{n}(-\mathbf{G}) .
\end{aligned}
$$

Essas paridades podem ser substituídas na equação (2.78) para o cálculo do invariante $\mathbf{Z}_{2}$.

\section{C.2 Invariante $\mathrm{Z}_{2}$ sem simetria de inversão}

No caso dos materiais que não possuem a simetria de inversão (não-centrossimétricos), o invariante topológico $\mathbf{Z}_{2}$ é calculado através do Pfaffiano da matriz $w$ nos pontos TRIM, como mostrado na equação (2.64).

A matriz $w$ na base de ondas planas é dada por

$$
\begin{aligned}
w_{i j}\left(\Gamma_{\alpha}\right) & =\left\langle u_{i,-\Gamma_{\alpha}}^{\sigma}|\Theta| u_{j, \Gamma_{\alpha}}^{\sigma^{\prime}}\right\rangle \\
& =\int d^{3} u_{i,-\Gamma_{\alpha}}^{* \sigma}(\mathbf{r}) \Theta u_{j, \Gamma_{\alpha}}^{\sigma^{\prime}}(\mathbf{r}) .
\end{aligned}
$$

Fazendo a transformação no espaço- $k$

$$
u_{i,-\Gamma_{\alpha}}^{\sigma *}(\mathbf{r})=\mathrm{e}^{-i 2 \Gamma_{\alpha} \cdot \mathbf{r}} u_{i, \Gamma_{\alpha}}^{\sigma *}(\mathbf{r})
$$

na equação (C.4), encontramos

$$
w_{i j}\left(\Gamma_{\alpha}\right)=\int d^{3} r \mathrm{e}^{-i 2 \Gamma_{\alpha} \cdot \mathbf{r}} u_{i, \Gamma_{\alpha}}^{\sigma *}(\mathbf{r}) \Theta u_{j, \Gamma_{\alpha}}^{\sigma^{\prime}}(\mathbf{r}) .
$$

Na base de $\operatorname{spin} s_{z}$ a equação (C.6) torna-se

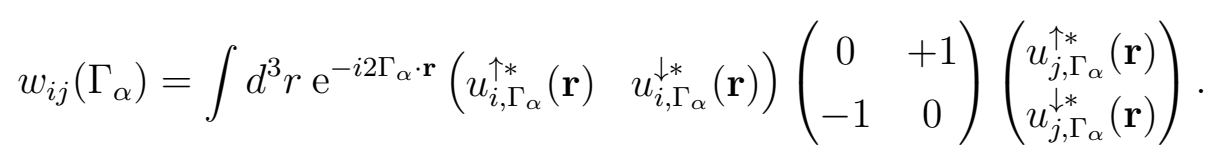

Usando a expansão em ondas planas (C.1) na equação (C.7), en- 
contramos

$$
w_{i j}\left(\Gamma_{\alpha}\right)=\frac{1}{\Omega} \sum_{\mathbf{G}, \mathbf{G}^{\prime}}\left[c_{i}^{\uparrow *}(\mathbf{G}) c_{j}^{\downarrow *}\left(\mathbf{G}^{\prime}\right)-c_{i}^{\downarrow *}(\mathbf{G}) c_{j}^{\uparrow *}\left(\mathbf{G}^{\prime}\right)\right] \int d^{3} r \mathrm{e}^{-i 2 \Gamma_{\alpha} \cdot \mathbf{r}} \mathrm{e}^{-i\left(\mathbf{G}+\mathbf{G}^{\prime}\right) \cdot \mathbf{r}}
$$

Finalmente, a integração da equação (C.8) fornece uma função delta de Kronecker, que quando efetuada a somatória em $\mathrm{G}^{\prime}$, chegamos em

$$
w_{i j}\left(\Gamma_{\alpha}\right)=\sum_{\mathbf{G}}\left[c_{i}^{\uparrow *}(\mathbf{G}) c_{j}^{\downarrow *}\left(-\mathbf{G}-2 \Gamma_{\alpha}\right)-c_{i}^{\downarrow *}(\mathbf{G}) c_{j}^{\uparrow *}\left(-\mathbf{G}-2 \Gamma_{\alpha}\right)\right]
$$

Essa expressão pode ser usada diretamente na equação (2.64) para calcular o invariante topológico $\mathrm{Z}_{2}$ na ausência da simetria de inversão. 


\section{Lista de publicações}

- Seixas, L., Abdalla, L.B., Schmidt, T.M. , Fazzio, A. \& Miwa, R.H., J. Appl. Phys. 113, 023705 (2013).

- Abdalla, L.B., Seixas, L., Schmidt, T.M., Miwa, R.H. \& Fazzio, A., Phys. Rev. B 88, 045312 (2013).

- Padilha, J.E., Seixas, L., Pontes, R.B., da Silva, A.J.R. \& Fazzio, A., Phys. Rev. B $88201106(\mathrm{R})$ (2013).

- Seixas, L., Padilha, J.E. \& Fazzio, A., Phys. Rev. B 89, 195403 (2014). 


\section{Bibliografia}

[1] Maxwell, J. C., A treatise on electricity and magnetism, volume 1, Clarendon press, 1873.

[2] Ashcroft, N. W. and Mermin, N. D., Solid State Physics, Harcourt College Publishers, 1976.

[3] Klitzing, K. v., Dorda, G., and Pepper, M., Phys. Rev. Lett. 45 (1980) 494.

[4] Thouless, D., Kohmoto, M., Nightingale, M., and Den Nijs, M., Phys. Rev. Lett. 49 (1982) 405.

[5] Laughlin, R. B., Phys. Rev. B 23 (1981) 5632.

[6] Halperin, B. I., Phys. Rev. B 25 (1982) 2185.

[7] Hatsugai, Y., Phys. Rev. Lett. 71 (1993) 3697.

[8] Avron, J. E., Seiler, R., and Simon, B., Phys. Rev. Lett. 51 (1983) 51.

[9] Dyakonov, M. and Perel, V., Phys. Lett. A 35 (1971) 459.

[10] Murakami, S., Nagaosa, N., and Zhang, S.-C., Science 301 (2003) 1348. 
[11] Kato, Y., Myers, R., Gossard, A., and Awschalom, D., Science 306 (2004) 1910.

[12] Kane, C. L. and Mele, E. J., Phys. Rev. Lett. 95 (2005) 226801.

[13] Bernevig, B. A., Hughes, T. L., and Zhang, S.-C., Science 314 (2006) 1757.

[14] König, M. et al., Science 318 (2007) 766.

[15] Moore, J. E. and Balents, L., Phys. Rev. B 75 (2007) 121306.

[16] Fu, L. and Kane, C. L., Phys. Rev. B 76 (2007) 045302.

[17] Hsieh, D. et al., Nature (London) 452 (2008) 970.

[18] Zhang, H. et al., Nature Phys. 5 (2009) 438.

[19] Xia, Y. et al., Nature Phys. 5 (2009) 398.

[20] Chen, Y. L. et al., Science 325 (2009) 178.

[21] Hasan, M. Z. and Kane, C. L., Rev. Mod. Phys. 82 (2010) 3045.

[22] Pesin, D. and MacDonald, A. H., Nature Mater. 11 (2012) 409.

[23] Fan, Y. et al., Nature Mater. (2014).

[24] Awschalom, D. and Samarth, N., Physics 2 (2009) 50.

[25] Žutić, I., Fabian, J., and Sarma, S. D., Rev. Mod. Phys. 76 (2004) 323.

[26] Fu, L. and Kane, C. L., Phys. Rev. Lett. 100 (2008) 096407.

[27] Nayak, C., Simon, S. H., Stern, A., Freedman, M., and Sarma, S. D., Rev. Mod. Phys. 80 (2008) 1083.

[28] Kittel, C., Introduction to Solid State Physics, John Wiley \& Sons, 1996. 
[29] Fazzio, A. and Watari, K., Introdução à Teoria de Grupos com Aplicações em Moléculas e Sólidos, Editora UFSM, 2009.

[30] Berry, M. V., Proc. R. Soc. Lond. A 392 (1984) 45.

[31] Xiao, D., Chang, M.-C., and Niu, Q., Rev. Mod. Phys. 82 (2010) 1959.

[32] Zak, J., Phys. Rev. Lett. 62 (1989) 2747.

[33] Simon, B., Phys. Rev. Lett. 51 (1983) 2167.

[34] Sakurai, J. J., Modern Quantum Mechanics, Addinson-Wesley Publishing Company, 1994.

[35] Fu, L. and Kane, C. L., Phys. Rev. B 74 (2006) 195312.

[36] King-Smith, R. and Vanderbilt, D., Phys. Rev. B 47 (1993) 1651.

[37] Kane, C. L. and Mele, E. J., Phys. Rev. Lett. 95 (2005) 146802.

[38] Aufray, B. et al., Appl. Phys. Lett. 96 (2010) 183102.

[39] Lalmi, B. et al., Appl. Phys. Lett. 97 (2010) 223109.

[40] Liu, C.-C., Feng, W., and Yao, Y., Phys. Rev. Lett. 107 (2011) 076802.

[41] Ando, Y., arXiv:1304.5693 (2013).

[42] Hohenberg, P. and Kohn, W., Phys. Rev. 136 (1964) B864.

[43] Kohn, W. and Sham, L. J., Phys. Rev. 140 (1965) A1 133.

[44] Kresse, G. and Furthmüller, J., Comp. Mat. Sci. 6 (1996) 15.

[45] Kresse, G. and Furthmüller, J., Phys. Rev. B 54 (1996) 11169.

[46] Ceperley, D. and Alder, B., Phys. Rev. Lett. 45 (1980) 566. 
[47] Perdew, J. P. and Zunger, A., Phys. Rev. B 23 (1981) 5048.

[48] Monkhorst, H. J. and Pack, J. D., Phys. Rev. B 13 (1976) 5188.

[49] Blöchl, P. E., Phys. Rev. B 50 (1994) 17953.

[50] Kresse, G. and Joubert, D., Phys. Rev. B 59 (1999) 1758.

[51] Rosengaard, N. and Skriver, H. L., Phys. Rev. B 47 (1993) 12865.

[52] Lee, B.-J., Shim, J.-H., and Baskes, M., Phys. Rev. B 68 (2003) 144112

[53] Qi, X.-L. and Zhang, S.-C., Rev. Mod. Phys. 83 (2011) 1057.

[54] Hsieh, D. et al., Nature (London) 460 (2009) 1101.

[55] Alpichshev, Z. et al., Phys. Rev. Lett. 104 (2010) 016401.

[56] Koumoulis, D. et al., Phys. Rev. Lett. 110 (2013) 026602.

[57] Schmidt, T. M., Miwa, R., and Fazzio, A., Phys. Rev. B 84 (2011) 245418.

[58] Abdalla, L., Seixas, L., Schmidt, T., Miwa, R., and Fazzio, A., Phys. Rev. B 88 (2013) 045312.

[59] Chen, Y. et al., Science 329 (2010) 659.

[60] Sato, T. et al., Nature Phys. 7 (2011) 840.

[61] Dycus, J. H., White, R. M., Pierce, J. M., Venkatasubramanian, R., and LeBeau, J. M., Appl. Phys. Lett. 102 (2013) 081601.

[62] Liu, X. et al., Appl. Phys. Lett. 99 (2011) 171903.

[63] Li, H. et al., Appl. Phys. Lett. 102 (2013) 074106. 
[64] Takagaki, Y., Jenichen, B., and Tominaga, J., Phys. Rev. B 87 (2013) 245302.

[65] Schröter, T. et al., Surf. Sci. 307-309, Part B (1994) 650 , Proceedings of the European Conference on Surface Science.

[66] Schmidt, W. G. and Bechstedt, F., Phys. Rev. B 50 (1994) 17280.

[67] Yao, Y., Ye, F., Qi, X.-L., Zhang, S.-C., and Fang, Z., Phys. Rev. B 75 (2007) 041401.

[68] Lin, C.-L. et al., Appl. Phys. Expr. 5 (2012) 045802.

[69] Feng, B. et al., Nano Lett. 12 (2012) 3507.

[70] Xu, Y. et al., Phys. Rev. Lett. 111 (2013) 136804.

[71] Padilha, J., Seixas, L., Pontes, R. B., da Silva, A. J., and Fazzio, A., Phys. Rev. B 88 (2013) 201106.

[72] Bianco, E. et al., ACS Nano 7 (2013) 4414.

[73] Singh, A. K. and Yakobson, B. I., Nano Lett. 9 (2009) 1540.

[74] de Almeida, J., Rocha, A., Singh, A. K., Fazzio, A., and da Silva, A. J., Nanotechnology 24 (2013) 495201.

[75] Ozaki, T., Phys. Rev. B 67 (2003) 155108.

[76] Perdew, J. P., Burke, K., and Ernzerhof, M., Phys. Rev. Lett. 77 (1996) 3865.

[77] Ezawa, M. and Nagaosa, N., Phys. Rev. B 88 (2013) 121401.

[78] Ezawa, M., New J. Phys. 14 (2012) 033003.

[79] Zunger, A., Wei, S.-H., Ferreira, L., and Bernard, J. E., Phys. Rev. Lett. 65 (1990) 353. 
[80] Wei, S.-H., Ferreira, L., Bernard, J. E., and Zunger, A., Phys. Rev. B 42 (1990) 9622.

[81] Venezuela, P., Dalpian, G., da Silva, A. J., and Fazzio, A., Phys. Rev. B 64 (2001) 193202.

[82] Thomas, L. H., Proc. Cambridge Phil. Soc. 23 (1927) 542.

[83] Fermi, E., Rend. Accad. Naz. Lincei 6 (1927) 602.

[84] Capelle, K., Brazillian Journal of Physics 36 (2006) 1318.

[85] Dirac, P. A. M., Proc. Cambridge Phil. Soc. 26 (1930) 376.

[86] von Weizsäcker, C. F., Z. Phys. 96 (1935) 431.

[87] Jones, R. O. and Gunnarsson, O., Rev. Mod. Phys. 61 (1989) 689.

[88] Teller, E., Rev. Mod. Phys. 34 (1962) 627.

[89] Dirac, P. A. M., Proc. R. Soc. Lond. A (1928) 610. 